\title{
Multiple Dirichlet Series and Moments of Zeta and $L$-Functions
}

\author{
ADRIAN DIACONU ${ }^{1}$, DORIAN GOLDFELD ${ }^{1}$ and JEFFREY HOFFSTEIN ${ }^{2}$ \\ ${ }^{1}$ Department of Mathematics, Columbia University, New York, NY 10027, U.S.A. \\ e-mail: \{cad, goldfeld $\} @$ math.columbia.edu \\ ${ }^{2}$ Department of Mathematics, Brown University, Providence, RI 02912, U.S.A. \\ e-mail: jhoff@math.brown.edu
}

(Received: 7 February 2002; accepted in final form: 27 August 2002)

\begin{abstract}
This paper develops an analytic theory of Dirichlet series in several complex variables which possess sufficiently many functional equations. In the first two sections it is shown how straightforward conjectures about the meromorphic continuation and polar divisors of certain such series imply, as a consequence, precise asymptotics (previously conjectured via random matrix theory) for moments of zeta functions and quadratic $L$-series. As an application of the theory, in a third section, we obtain the current best known error term for mean values of cubes of cent ral values of Dirichlet $L$-series. The methods utilized to derive this result are the convexity principle for functions of several complex-variables combined with a knowledge of groups of functional equations for certain multiple Dirichlet series.
\end{abstract}

Mathematics Subject Classifications (2000). 11M06, Secondary: 111F66, $11 \mathrm{M} 41$.

Key words. $L$-functions, moments, multiple Dirichlet series, twists, zeta functions.

\section{Introduction}

A Dirichlet series of type

$$
\sum_{m_{1}=1}^{\infty} \cdots \sum_{m_{n}=1}^{\infty} \frac{1}{m_{1}^{S_{1}} \cdots m_{1}^{S_{n}}} \int_{0}^{\infty} \cdots \int_{0}^{\infty} a\left(m_{1}, \ldots, m_{n}, t_{1}, \ldots, t_{\ell}\right) t_{1}^{-w_{1}} \cdots t_{1}^{-w_{\ell}} \mathrm{d} t_{1} \cdots \mathrm{d} t_{\ell}
$$

(where $a\left(m_{1}, \ldots, m_{n}, t_{1}, \ldots, t_{\ell}\right)$ is a complex-valued smooth function) will be called a multiple Dirichlet series. It can be viewed as a Dirichlet series in one variable whose coefficients are again Dirichlet series in several other variables. One of the simplest examples of a multiple Dirichlet series of more than one variable is given by

$$
\sum_{d}^{\infty} \frac{L\left(s, \chi_{d}\right)}{|d|^{w}}
$$


where the sum ranges over fundamental discriminants of quadratic fields, $\chi_{d}$ is the quadratic character associated to these fields, and

$$
L\left(s, \chi_{d}\right)=\sum_{n=1}^{\infty} \frac{\chi_{d}(n)}{n^{s}}
$$

is the classical Dirichlet $L$-function. This type of double Dirichlet series and a method to obtain its analytic continuation first appeared in a paper of Siegel $[\mathrm{S}]$ in 1956. More generally, one may consider

$$
Z\left(s_{1}, s_{2}, \ldots, s_{m}, w\right)=\sum_{d} \frac{L\left(s_{1}, \chi_{d}\right) \cdot L\left(s_{2}, \chi_{d}\right) \cdots L\left(s_{m}, \chi_{d}\right)}{|d|^{w}} .
$$

Multiple Dirichlet series arise naturally in many contexts and have been the subject of a number of papers in the recent past. See, [B-F-H-2] for an overview and references. The reason for their interest is most apparent when they take the form (1.1). It is easy to see that if, for fixed $s_{1}, s_{2}, \ldots, s_{m}$, the analytic continuation of $Z\left(s_{1}, s_{2}, \ldots, s_{m}, w\right)$ could be obtained to all $w \in \mathbb{C}$ then standard Tauberian arguments could be used to obtain information about the behavior of $L\left(s_{1}, \chi_{d}\right)$. $L\left(s_{2}, \chi_{d}\right) \cdots L\left(s_{m}, \chi_{d}\right)$ as $d$ varies. For example, mean values could be obtained if there is a pole at $w=1$. The situation becomes even more interesting when it is noted that quadratic twists of the $L$-series of automorphic forms on GL $(m)$ can be viewed as special cases of the product $L\left(s_{1}, \chi_{d}\right) \cdot L\left(s_{2}, \chi_{d}\right) \cdots L\left(s_{m}, \chi_{d}\right)$. The first example of this type of application that we are aware of is $[\mathrm{G}-\mathrm{H}]$ in the case $m=1$. Here mean value results are obtained for quadratic Dirichlet $L$-series. Similar results over a function field are obtained in [H-R], and recently, over more general function field, in [F-F]. Examples of the cases $m=2,3$ when the numerator is the $L$-series associated to a $\mathrm{GL}(m)$ cusp form are given in [B-F-H-2], [B-F-H-1].

In all these examples (except for [F-F]), the analytic continuation of (1.1) was obtained by treating the variable $w$ separately. The fact that the $L$-series or products of $L$-series in the numerator occurred in the Fourier coefficients of certain metaplectic Eisenstein series was exploited, and analytic continuation in $w$ was achieved by the application of Rankin-Selberg transforms.

It later became apparent, however, that there were many advantages to viewing multiple Dirichlet series as functions of several complex variables. In particular, consider (1.1) but 'improve' it by redefining the $L$-series in such a way that $\prod_{i=1}^{m} L\left(s_{i}, \chi_{d}\right)$ is the usual product of $L$-series if $d$ is (the square free part of) a fundamental discriminant, and is $\prod_{i=1}^{m} L\left(s_{i}, \chi_{d_{0}}\right)$ times a correction factor if $d$ is a square multiple of the square free part $d_{0}$. The correction factors are Dirichlet polynomials with functional equations and will be discussed further in Section 4.

The improved, or 'perfect' series, $Z^{*}\left(s_{1}, s_{2}, \ldots, s_{m}, w\right)$, then possesses some unexpected properties. In particular, in addition to the obvious functional equations sending $s_{i} \rightarrow 1-s_{i}, i=1, \ldots, m$, there are some 'hidden' functional equations that correspond to some surprising structure when the order of summation in $Z^{*}$ is altered. 
The fact that such a phenomenon can occur was first observed by Bump and Hoffstein in the case of $m=1$ and a rational function field, and is mentioned in $[\mathrm{H}]$. It was first observed and applied in the case $m=2$ in [F-H]. The possibility of using these extra functional equations as a basis for obtaining the analytic continuation of double Dirichlet series was then discussed in [B-F-H-2]. It was observed there that in the cases where the numerator is an $L$-series of an automorphic form on $\mathrm{GL}(m)$, if $m=1,2$ or 3 then the functional equations of the corresponding perfect double Dirichlet series generate a finite group. It was also noted that by applying these functional equations to the region of absolute convergence a collection of overlapping regions was obtained whose convex hull was $\mathrm{C}^{2}$. Thus by appealing to a well known theorem in the theory of functions of several complex variables, the complete analytic continuation of $Z^{*}$ could be obtained.

In later work, [B-F-H-1], it was observed that a uniqueness principle operated in the cases $m=1,2,3$ and the correction factors were determined by, and could be computed from, the functional equations of $Z^{*}$. Curiously, for $m \geqslant 4$ the group of functional equations becomes infinite and simultaneously the uniqueness principle fails. The space of local solutions becomes 1 dimensional in the case $m=4$, and higher for $m>4$. This appears to correspond to an inability to analytically continue the double Dirichlet series past a curve of essential singularities. See [B-F-H-1,2] for further details. The paper of [F-F], in addition to providing a completely general analysis of the case $m=1$ over a function field, contains some further insights into this curious phenomenon.

We shall call a multiple Dirichlet series (of $n$ complex variables) perfect if it has meromorphic continuation to $\mathbb{C}^{n}$ and, in addition, it satisfies a group of functional equations. The case $m=3$ is thus of great interest as the last instance in which the perfect multiple Dirichlet series (for the family of quadratic Dirichlet $L$-functions) are understood completely. In [B-F-H-1] a description of the 'good' correction factors was obtained for the case of $m=3$ and an arbitrary automorphic form $f$ on GL(3). These are the factors corresponding to primes not dividing 2 or the level of $f$. This information was then used to obtain the analytic continuation of the associated perfect double Dirichlet series. As a consequence, non-vanishing results for quadratic twists of $L\left(1 / 2, f, \chi_{d}\right)$ were obtained. Also, after taking a residue at $w=1$, a new proof was obtained for the analytic continuation of the symmetric square of an automorphic form on GL(3).

One purpose of this paper is to apply the ideas of [B-F-H-1] to obtain the meromorphic continuation of the series $Z^{*}(s, s, s, w)$. After obtaining this and developing a sieving method analogous to that used in [G-H] we reconstruct the unimproved series of (1.1). Applying the analytic properties of this we prove the following

THEOREM 1.1. For $d$ summed over fundamental discriminants, and any $\epsilon>0$

$$
\sum_{|d| \leqslant x} L\left(\frac{1}{2}, \chi_{d}\right)^{3}\left(1-\frac{|d|}{x}\right)=\frac{1}{2} \cdot \frac{6}{\pi^{2}} a_{3} \cdot \frac{1}{2880} \cdot x(\log x)^{6}+\sum_{i=0}^{5} c_{i} x(\log x)^{i}+\mathcal{O}_{\epsilon}\left(x^{\frac{-1}{\epsilon}+\epsilon}\right) .
$$


The constants $c_{i}$ are effectively computable. The following unweighted estimate also holds:

$$
\sum_{|d| \leqslant x} L\left(\frac{1}{2}, \chi_{d}\right)^{3}=\frac{6}{\pi^{2}} a_{3} \cdot \frac{1}{2880} \cdot x(\log x)^{6}+\sum_{i=0}^{5} d_{i} x(\log x)^{i}+\mathcal{O}_{\epsilon}\left(x^{\theta+\epsilon}\right),
$$

where the constants $d_{i}$ are also effectively computable and $\theta=\frac{1}{36}(47-\sqrt{265}) \sim$ $0.853366 \ldots$

This improves on Soundararajan's [So], bound of $\mathrm{O}\left(x^{\frac{11}{12}+\epsilon}\right)$. The weight $(1-(|d| / x))$ is included in the first part to show the optimal error term obtainable by this method. It will be shown in Section 4.4, Proposition 4.12, that we expect the multiple Dirichlet series $Z^{*}\left(\frac{1}{2}, \frac{1}{2}, \frac{1}{2}, w\right)$ to have an additional simple pole at $w=\frac{3}{4}$ with non-zero residue. Note that the function $Z^{*}\left(\frac{1}{2}, \frac{1}{2}, \frac{1}{2}, w\right)$ is perfect and has meromorphic continuation everywhere. It is unclear whether $Z\left(\frac{1}{2}, \frac{1}{2}, \frac{1}{2}, w\right)$ has a pole at $w=\frac{3}{4}$, assuming it has meromorphic continuation to $\Re(w)>\frac{3}{4}-\epsilon$ for some $\epsilon>0$. We expect this matter to be clarified shortly by computations being done by Q. Zhang.

Remark. In general, for higher moments, careful analysis of Sections 2.3 and 3.1 can be used to obtain all the coefficients of the polynomial in $\log x$ of the main term in the asymptotic formula.

The major objective of this paper is to, at least conjecturally, pass the barrier of $m \geqslant 4$. The first obstacle to accomplishing this is our incomplete understanding of the correct form of the class of perfect multiple Dirichlet series for $m \geqslant 4$. There is an infinite family of choices, every member of which possesses the correct functional equations. However, for any one of these choices, if an analytic continuation could be obtained to a neighborhood including the point $(1 / 2,1 / 2, \ldots, 1 / 2,1)$ then a sieving argument could be applied and a formula analogous to Theorem 1.1 could be proved. In particular, this would imply the truth of Conjecture 3.1 of Conrey, Farmer, Keating, and Snaith giving the precise asymptotics for the moments of $\sum_{|d| \leqslant x} L\left(1 / 2, \chi_{d}\right)^{m}$ for $m=1,2,3, \ldots$ In [B-F-H-2] it is explained how if the variables are specialized to $s=s_{1}=\cdots=s_{m}$, then any multiple Dirichlet series possessing the correct functional equations must hit a certain curve of essential singularities. A similar hypercurve is encountered for $m \geqslant 4$ when the variables are not specialized. However, the point $(1 / 2,1 / 2, \ldots, 1 / 2,1)$ lies well inside the boundary of this curve. Another way of saying this is that by taking the area of absolute convergence of a corrected analog of (1.1) and applying the infinite group of functional equations a region of analytic continuation is obtained. For $m \geqslant 4$ the point $(1 / 2,1 / 2, \ldots, 1 / 2,1)$ lies outside this region, but inside the region contained by the curve of essential singularities. The case $m=4$ is particularly intriguing, as $(1 / 2,1 / 2,1 / 2,1 / 2,1)$ lies right on the edge of the open hyperplane of analytic continuation that can be obtained. 
In Section 3 we make the reasonable assumption that an analytic continuation exists past the point $(1 / 2,1 / 2, \ldots, 1 / 2,1)$ for a corrected analog of $(1.1)$. We then calculate the contribution of the $2^{m}$ polar divisors of (1.1) that pass through this point. This gives us a description of the whole principle part in the Laurent expansion of (1.1) around this point. This description is then translated into Conjecture 3.1.

As far as the present authors are aware, the first examples of multiple Dirichlet series involving integrals appear in the paper of A. Good [G] first announced in 1984. Let $f(z)$ be a holomorphic cusp form of even weight $k$ for the modular group $\Gamma=\operatorname{SL}(2, Z)$. By developing an ingenious generalization of the Rankin-Selberg convolution in polar coordinates Good obtained the meromorphic continuation of the multiple Dirichlet series

$$
\int_{1}^{\infty}\left|L_{f}\left(\frac{k}{2}+i t\right)\right|^{2} t^{-w} \mathrm{~d} t
$$

where $L_{f}(s)$ is the Hecke $L$-function associated to $f$ by Mellin transform. He showed that this function has at most simple poles at $w=\frac{1}{2}+i r$, where $w=\frac{1}{4}+r^{2}$ is an eigenvalue associated to a Maass form on $\Gamma$. Good $[G]$ even showed how to introduce weighting factors into the integral which gave a functional equation in $w$. His method can also be extended to obtain the meromorphic continuation of

$$
\int_{1}^{\infty} L_{f}\left(s_{1}+i t\right) L_{f}\left(s_{2}-i t\right) t^{-w} \mathrm{~d} t
$$

In Section 2, we develop the theory of multiple Dirichlet series associated to moments of the Riemann zeta function. In this case, the perfect object has been found for $m=2$ (using theta functions) and for $m=4$ (using Eisenstein series) by Good $[\mathrm{G}]$, but his theory has never been fully worked out. We consider the multiple Dirichlet series

$$
Z\left(s_{1}, \ldots, s_{2 m}, w\right)=\int_{1}^{\infty} \zeta\left(s_{1}+i t\right) \cdots \zeta\left(s_{m}+i t\right) \cdot \zeta\left(s_{m+1}-i t\right) \cdots \zeta\left(s_{2 m}-i t\right) t^{-w} \mathrm{~d} t
$$

and show that it has meromorphic continuation (as a function of $2 m+1$ complex variables) slightly beyond the region of absolute convergence given by $\mathfrak{R}\left(s_{i}\right)>1, \mathfrak{R}(w)>1(i=1,2, \ldots, 2 m)$ with a polar divisor at $w=1$. We also show that $Z\left(s_{1}, \ldots, s_{2 m}, w\right)$ satisfies certain quasi-functional equations (see Section 2.2) which allows one to meromorphically continue the multiple Dirichlet series to an even larger region. It is proved (subject to Conjecture 2.7) that $Z\left(\frac{1}{2}, \ldots, \frac{1}{2}, w\right)$ has a multiple pole at the point $w=1$, and the leading coefficient in the Laurent expansion is computed explicitly in Proposition 2.9. Under the assumption that $Z\left(\frac{1}{2}, \ldots, \frac{1}{2}, w\right)$ has holomorphic continuation to the region $\operatorname{Re}(w) \geqslant 1$ (except for the multiple pole at $w=1$, we derive the Conrey-Ghosh-Keating-Snaith conjecture (see [Ke-Sn-1] and [C-Gh-2]) for the ( $2 m)$ th moment of the zeta function as predicted by random matrix theory. 
Recently [CFKRS] have presented a heuristic method via approximate functional equations for obtaining moment conjectures for integral as well as real and complex moments for general families of zeta and L-functions. Their method is related to ours in that it uses a group of approximate functional equations in several complex variables.

Remark. Notice that the methods presented in this paper do not require that the $L$-functions (for which we want to obtain moments) have an Euler product. It is only necessary that they have meromorphic continuation and satisfy a group of functional equations.

\section{Moments of the Riemann Zeta-Function}

For $\Re>1$, let

$$
\zeta(s)=\sum_{n=1}^{\infty} \frac{1}{n^{s}}=\prod_{p}\left(1-\frac{1}{p^{s}}\right)^{-1}
$$

denote the Riemann zeta function which has meromorphic continuation to the whole complex plane with a single simple pole at $s=1$ with residue 1 . It is well known (see Titchmarsh [T] that $\zeta$ satisfies the functional equation $\zeta(s)=\chi(s) \zeta(1-s)$ where

$$
\chi(1-s)=\frac{1}{\chi(s)}=2(2 \pi)^{-s} \cos \left(\frac{\pi s}{2}\right) \Gamma(s) .
$$

In 1918 Hardy and Littlewood [H-L] obtained the second moment

$$
\int_{0}^{x}\left|\zeta\left(\frac{1}{2}+i t\right)\right|^{2} \mathrm{~d} t \sim x \log x
$$

and in 1926 Ingham [I] obtained the fourth moment

$$
\int_{0}^{x}\left|\zeta\left(\frac{1}{2}+i t\right)\right|^{4} \mathrm{~d} t \sim \frac{1}{2 \pi^{2}} x(\log x)^{4} .
$$

This result was not significantly improved until the work of Heath and Brown [H-B] in 1979 where it was shown that

$$
\int_{0}^{x}\left|\zeta\left(\frac{1}{2}+i t\right)\right|^{4} \mathrm{~d} t=x \cdot P_{4}(\log x)+\mathcal{O}\left(x^{\frac{7}{8}+\epsilon}\right),
$$

where $P_{4}$ is a certain polynomial of degree four. More recently, Zavorotny [Z] (1989), and by a different method, Motohashi [Mot1] (1993) has proved that

$$
\int_{0}^{x}\left|\zeta\left(\frac{1}{2}+i t\right)\right|^{4} \mathrm{~d} t=x \cdot P_{4}(\log x)+\mathrm{O}\left(x^{\frac{2}{3}+\epsilon}\right)
$$


Motohashi's work was based on earlier work of Atkinson [A]. By a careful analysis of the Kuznetsov trace formula, Motohashi [Mot2] introduced and was able to obtain the meromorphic continuation (in $w$ ) of the function

$$
\int_{1}^{\infty} \zeta(s+i t)^{2} \zeta(s-i t)^{2} t^{-w} \mathrm{~d} t
$$

Motohashi pointed out that it is, therefore, possible to view the Riemann zeta function as a generator of Maass wave form $L$-functions. Motohashi [Mot3] has generalized his methods to a wide variety of cases which include mean square and fourth moment of the Riemann zeta function, mean square of Hecke $L$-series attached to holomorphic cusp forms, and mean square of quadratic zetas. These should be compared to the earlier work of Good [G].

There has been a longstanding folklore conjecture that

$$
\int_{0}^{x}\left|\zeta\left(\frac{1}{2}+i t\right)\right|^{2 k} \mathrm{~d} t \sim c_{k} x(\log x)^{k^{2}}
$$

In 1984 Conrey and Ghosh [C-Gh-2] gave the more precise conjecture that

$$
c_{k}=\frac{g_{k} a_{k}}{\Gamma\left(1+k^{2}\right)},
$$

where

$$
a_{k}=\prod_{p}\left(1-\frac{1}{p}\right)^{k^{2}} \sum_{j=0}^{\infty} \frac{d_{k}\left(p^{j}\right)^{2}}{p^{j}}
$$

is the arithmetic factor and $g_{k}$, an integer, is a geometric factor. Here, $d_{k}(n)$ denotes the number of representations of $n$ as a product of $k$ positive integers. In this notation, the result of Hardy and Littlewood states that $g_{1}=1$, while Ingham's result is that $g_{2}=2$. In 1998, Conrey and Ghosh [C-Gh-1] conjectured that $g_{3}=42$, and more recently in 1999, Conrey and Gonek [C-G] conjectured that $g_{4}=24024$. Up to this point, using classical techniques based on approximating $\zeta(s)$ by Dirichlet polynomials, there seemed to be no way to conjecture the value of $g_{k}$ in general.

In accordance with the philosophy of Katz and Sarnak [K-S] that one may associate probability spaces over compact classical groups to families of zeta and L-functions, Keating and Snaith [Ke-Sn-2] (see also [B-H]) computed moments of characteristic polynomials of matrices in the unitary group $U(n)$ and formulated the conjecture that

$$
g_{k}=k^{2} ! \prod_{j=0}^{k-1} \frac{j !}{(j+k) !}
$$

for any positive integer $k$. This conjecture agreed with all the known results and was strongly supported by numerical computations.

We show in the next sections that there exists a multiple Dirichlet series of several complex variables of the type (2.2) previously introduced by Motohashi, with a polar divisor at $w=1$, whose residue is simply related to the constants (2.4), (2.5), (2.6). We 
further show that if one could holomorphically continue this multiple Dirichlet series slightly beyond this polar divisor, a proof of the Conrey-Ghosh-Keating-Snaith conjecture would follow.

\subsection{THE MULTIPLE DIRICHLET SERIES FOR THE RIEMANN ZETA FUNCTION}

Let $s_{1}, s_{2}, \ldots, s_{2 m}, w$ denote complex variables, $k$ be an integer, and $\epsilon_{i}= \pm 1$ for $i=1,2, \ldots, 2 m$. We shall consider multiple Dirichlet series of type

$$
\begin{aligned}
& Z_{\epsilon_{1}, \ldots, \epsilon_{2 m}, k}\left(s_{1}, \ldots, s_{2 m}, w\right) \\
& =\int_{1}^{\infty} \zeta\left(s_{1}+\epsilon_{1} i t\right) \cdots \zeta\left(s_{2 m}+\epsilon_{2 m} i t\right)\left(\frac{2 \pi e}{t}\right)^{k i t} t^{-w} \mathrm{~d} t .
\end{aligned}
$$

It is easy to see that the integral in (2.7) converges absolutely for $\Re(w)>1$ and $\Re\left(s_{i}\right)>1,(i=1,2, \ldots, 2 m)$, and defines (in this region) a holomorphic function of $2 m+1$ complex variables. These series are more general than the series (2.2) introduced by Motohashi in that they contain the factor $(2 \pi e / t)^{k i t}$. It will be shortly seen that this factor occurs naturally because of the asymptotic formulae [T]

$$
\begin{aligned}
& \chi(s+i t)=\mathrm{e}^{\frac{i \pi}{4}}\left(\frac{2 \pi}{t}\right)^{s-\frac{1}{2}}\left(\frac{2 \pi e}{t}\right)^{i t}\left\{1+\mathrm{O}\left(\frac{1}{t}\right)\right\}, \\
& \chi(s+i t)=\mathrm{e}^{\frac{-i \pi}{4}}\left(\frac{2 \pi}{t}\right)^{s-\frac{1}{2}}\left(\frac{2 \pi e}{t}\right)^{-i t}\left\{1+\mathrm{O}\left(\frac{1}{t}\right)\right\} \text { (for fixed } s \text { and } t \rightarrow \infty \text { ), }
\end{aligned}
$$

for $\chi$, the function occurring in the functional Equation (2.1) for the Riemann zeta function.

PROPOSITION 2.1. For $\sigma>0$, the function $Z_{\epsilon_{1}, \ldots, \epsilon_{2 m}, k}\left(s_{1}, \ldots, s_{2 m}, w\right)$ can be holomorphically continued to the domain $\Re\left(s_{i}\right)>-\sigma($ for $i=1, \ldots, 2 m)$ and $\Re(w)>1+2 m\left(\frac{1}{2}+\sigma\right)$. Furthermore, for $k \neq 0, Z_{\epsilon_{1}, \ldots, \epsilon_{2 m}, k}$ can be holomorphically continued for $\Re(w)>0$ and $\Re\left(s_{i}+(w /|k|)\right)>1+|k|^{-1}(i=1, \ldots, 2 m)$, and for $k=0$, it can be meromorphically continued for $\Re(w)>0$ and $\Re\left(s_{i}\right)>1(i=1, \ldots, 2 m)$ with a single simple pole at $w=1$ with residue

$$
\sum_{\substack{\ell_{1}, \ldots, \ell_{2 m} \\ \ell_{1}^{\epsilon} \ldots \ell_{2 m}^{2 m}=1}} \ell_{1}^{-s_{1}} \cdots \ell_{2 m}^{-s_{2 m}}
$$

Proof. The first part of the Proposition follows immediately from the well-known convexity bound

$$
|\zeta(s+i t)| \ll_{s}(1+|t|)^{\frac{1}{2}+\sigma},
$$


for $\Re(s)>-\sigma$, where the implied constant depends at most on $s$. For the second part, we need the following lemma.

LEMMA 2.2 Let $B>0$ and $k \in \mathbb{R}$ be fixed. For $\Re(w)>1$ the integral

$$
\left|I_{B, k}(w)\right|=\int_{1}^{\infty} B^{i t}\left(\frac{2 \pi e}{t}\right)^{k i t} t^{-w} \mathrm{~d} t
$$

converges absolutely and defines a holomorphic function of $w$. Further, for $\{B, k\} \neq\{1,0\}$, the function $I_{B, k}(w)$ may be holomorphically continued to $\Re(w)>0$, and for $0<\Re(w) \leqslant 1$, it satisfies the bound

$$
\left|I_{B, k}(w)\right| \ll_{k, w} \begin{cases}\frac{1}{|\log B|} & \text { if } \quad k=0, \\ 1+B^{\frac{1-\Re(w)}{k}}(1+|\log B|) & \text { if } \quad k \neq 0 .\end{cases}
$$

Finally, when $B-1, k=0$, we have $I_{1,0}(w)=1 /(w-1)$.

Proof. First, a simple computation shows that $I_{1,0}(w)=1 /(w-1)$. Also, integrating by parts, it can easily be seen that $I_{B, 0}(w)$ is a holomorphic function for $\Re(w)>0$. In this case, we have the estimate

$$
\left|I_{B, 0}(w)\right| \ll_{w} \frac{1}{|\log B|} .
$$

For $k \neq 0$ and $B^{\frac{1}{k}} \geqslant(2 \pi)^{-1}$, we split the integral defining $I_{B, k}$ into two parts

$$
I_{B, k}(w)=\int_{1}^{\frac{A+1}{e}}\left(\frac{A}{t}\right)^{k i t} t^{-w} \mathrm{~d} t+\int_{\frac{A+1}{e}}^{\infty}\left(\frac{A}{t}\right)^{k i t} t^{-w} \mathrm{~d} t
$$

where $A=2 \pi e \cdot B^{\frac{1}{k}}$. We estimate the first integral trivially, so, for $0<\Re(w) \leqslant 1$,

$$
\begin{aligned}
\left|\int^{\frac{A+1}{e}}\left(\frac{A}{t}\right)^{k i t} t^{-w} \mathrm{~d} t\right| & <\frac{\left(\frac{A+1}{e}\right)^{1-\Re(w)}-1}{1-\Re(w)}<\left(\frac{A+1}{e}\right)^{1-\Re(w)} \log \left(\frac{A+1}{e}\right) \\
& \ll k, w B^{\frac{1-\Re(w)}{k}}(1+|\log B|) .
\end{aligned}
$$

Now, integrating by parts, we have

$$
\begin{aligned}
\int_{\frac{A+1}{e}}^{\infty}\left(\frac{A}{t}\right)^{k i t} t^{-w} \mathrm{~d} t= & \int_{\frac{A+1}{e}}^{\infty}\left(\frac{A}{t}\right)^{k i t} i k(\log A-\log t-1) \cdot \frac{1}{i k(\log A-\log t-1) t^{w}} \mathrm{~d} t \\
= & \frac{1}{i k} \cdot \frac{\left(\frac{\mathrm{e} A}{A+1}\right)^{\frac{k i(A+1)}{e}}}{\log \left(1+\frac{1}{A}\right)} \cdot \frac{\mathrm{e}^{w}}{(A+1)^{w}}-\frac{1}{i k} \int_{\frac{A+1}{e}}^{\infty}\left(\frac{A}{t}\right)^{k i t} \times \\
& \times \frac{1}{(\log A-\log t-1)^{2} t^{w+1}} \mathrm{~d} t+ \\
& +\frac{w}{i k} \int_{\frac{A+1}{e}}^{\infty}\left(\frac{A}{t}\right)^{k i t} \cdot \frac{1}{(\log A-\log t-1) t^{w+1}} \mathrm{~d} t
\end{aligned}
$$


It follows that the last two integrals converge absolutely for $\Re(w)>0$, and hence, the function $I_{H, k}$ is holomorphic in this region. Moreover, we have the estimate

$$
\begin{aligned}
\left|\int_{\frac{+1}{e}}^{\infty}\left(\frac{A}{t}\right)^{k i t} t^{-w} \mathrm{~d} t\right| & \ll \\
& +\mid \frac{\mathrm{e}^{\Re(w)} A^{1-\Re(w)}}{|k|}+\int_{\frac{A+1}{e}}^{\infty} \frac{1}{\log \left(\frac{t e}{A}\right)} \cdot \frac{1}{t^{1+\Re(w)}} \mathrm{d} t+\frac{1}{|k|} \int_{\frac{A+1}{e}}^{\infty} \frac{1}{\log ^{2}\left(\frac{t e}{A}\right)} \cdot \frac{1}{t^{1+\Re(w)}} \mathrm{d} t \\
& \ll \frac{|w|}{\Re(w)} \cdot \frac{\mathrm{e}^{\Re(w)} A^{1-\Re(w)}}{|k|}+\frac{\mathrm{e}^{\Re(w)}}{|k| A^{\Re(w)}} \int_{1+\frac{1}{A}}^{\infty} \frac{1}{\log ^{2} u} \cdot \frac{1}{u^{1+\Re(w)}} \mathrm{d} u \\
& \ll \frac{|w|}{\Re(w)} \cdot \frac{\mathrm{e}^{\Re(w)} A^{1-\Re(w)}}{|k|} \ll_{k, w} B^{1-\Re(w)}
\end{aligned}
$$

which combined with the previous one gives the required bound for the function $I_{B, k}$. For the remaining case, $B^{\frac{1}{k}}<(2 \pi)^{-1}$, we split once again the integral into two parts

$$
I_{B, k}(w)=\int_{1}^{1+\frac{1}{e}}\left(\frac{A}{t}\right)^{k i t} t^{-w} \mathrm{~d} t+\int_{1+\frac{1}{e}}^{\infty}\left(\frac{A}{t}\right)^{k i t} t^{-w} \mathrm{~d} t
$$

A similar argument implies that the second integral converges absolutely for $\Re(w)>0$, and that $\left|I_{B, k}(w)\right| \ll_{k, w} 1$.

We now return to the proof of Proposition 2.1. For $\Re\left(s_{i}\right)>1 \quad(i=1, \ldots, 2 m)$,

$$
Z_{\epsilon_{1}, \ldots, \epsilon_{2 m}, k}\left(s_{1}, \ldots, s_{2 m}, w\right)=\sum_{\ell_{1}, \ldots, \ell_{2 m}} \ell_{1}^{-s_{i}} \cdots \ell_{2 m}^{-s 2 m} \int_{1}^{\infty}\left(\ell_{1}^{\epsilon_{1}} \cdots \ell_{2 m}^{\epsilon_{2 m}}\right)^{i t}\left(\frac{2 \pi e}{t}\right)^{k i t} t^{-w} \mathrm{~d} t
$$

where the sum ranges over all $2 m$-tuples $\left\{\ell_{1}, \ldots, \ell_{2 m}\right\}$ of positive integers. For $k \neq 0$ and $0<\Re(w) \leqslant 1$, it is clear that the series on the right side of (2.9) is absolutely convergent provided $\mathfrak{R}\left(s_{i}\right)(i=1, \ldots, 2 m)$ are sufficiently large. In fact, the estimates from Lemma 2.2 imply that we have absolute convergence even for $\Re\left(s_{i}+\frac{w}{|k|}\right)>1+|k|^{-1}(i=1, \ldots, 2 m)$. For $k=0$, we break the sum on the right side of (2.9) into two parts

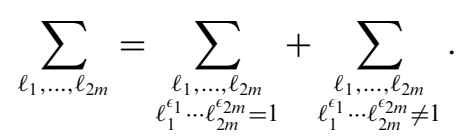

By Lemma 2.2 it immediately follows that the first sum in (2.10) will contribute a pole at $w=1$ with residue precisely as stated in Proposition 2.1. It is also clear from Lemma 2.2 that the second sum in (2.10) will give a holomorphic contribution to (2.9) provided $\Re\left(s_{i}\right)(i=1, \ldots, 2 m)$ are sufficiently large so that the sum over $\ell_{1}, \ldots, \ell_{2 m}$ converges absolutely. To show convergence for $\mathfrak{R}\left(s_{i}\right)>1$, $(i=1, \ldots, 2 m)$, is more delicate and we give the details. 
It follows from Lemma 2.2 that for $\mathfrak{R}\left(s_{i}\right)=\sigma>1,(i=1, \ldots, 2 m)$,

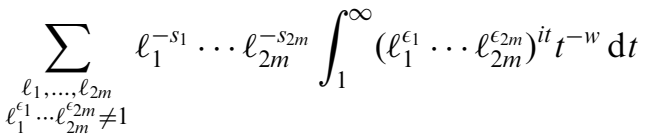

$$
\begin{aligned}
& \ll_{w} \sum_{\substack{\ell_{1}, \ldots, \ell_{2 m} \\
\ell_{1} \ldots \ell_{2 m}^{2 m} \neq 1}} \frac{1}{\left(\ell_{1} \cdots \ell_{2 m}\right)^{\sigma}} \frac{1}{\mid \log \ell_{1}^{\epsilon_{1}} \cdots \ell_{2 m}^{\epsilon_{2 m} \mid}} .
\end{aligned}
$$

We now break the sum on the right side of (2.11) into two parts

$$
\sum_{\substack{\ell_{1}, \ldots, \ell_{2 m} \\ \ell_{1}^{\epsilon_{1} \ldots \ell_{2 m}} \neq 1}}=\sum_{\substack{\ell_{\ell_{1}, \ldots, \ell_{2}} \\ \ell_{1}^{\epsilon_{1} \ldots \ell_{2 m}} \in\left(0, \frac{1}{2}(\cup) 2, \infty\right)}}+\sum_{\substack{\ell_{1}, \ldots, \ell_{2 m} \\ \ell_{1}^{\epsilon_{1} \ldots \ell_{2 m} m} \in\left(\frac{1}{2}, 1\right) \cup(1,2)}}
$$

The first series on the right side of (2.12) is obviously convergent for $\sigma>1$. We shall show that the second one is also convergent.

Without loss of generality, let us write

$$
\ell_{1}^{\epsilon_{1}} \cdots \ell_{2 m}^{\epsilon_{2 m}}=\frac{\ell_{1} \cdots \ell_{r}}{\ell_{r+1} \cdots \ell_{2 m}}
$$

It follows, upon setting $\ell_{1} \cdots \ell_{r}=k, \ell_{r+1} \cdots \ell_{2 m}=k \pm a$, that

$$
\begin{aligned}
& \sum_{\substack{\ell_{1}, \ldots, \ell_{2 m} \\
\frac{\ell_{1} \cdots r}{\ell_{r+1} \cdots \ell_{2 m}} \in\left(\frac{1}{2}, 1\right) \cup(1,2)}} \frac{1}{\left(\ell_{1} \cdots \ell_{2 m}\right)^{\sigma}} \frac{1}{\left|\log \frac{\ell_{1} \cdots \ell_{r}}{\ell_{r+1} \cdots \ell_{2 m}}\right|} \\
& =\sum_{k=2}^{\infty} \frac{d_{r}(k)}{k^{\sigma}} \sum_{a=1}^{k-1} \frac{d_{2 m-r}(k+a)}{(k+a)^{\sigma}} \cdot \frac{1}{\log \left(1+\frac{a}{k}\right)}- \\
& -\sum_{k=3}^{\infty} \frac{d_{r}(k)}{k^{\sigma}} \sum_{a=1}^{\left[\frac{k}{2}\right]} \frac{d_{2 m-r}(k-a)}{(k-a)^{\sigma}} \cdot \frac{1}{\log \left(1-\frac{a}{k}\right)} \ll \sum_{k=2}^{\infty} \frac{d_{r}(k)}{k^{\sigma}} \sum_{a=1}^{k-1} \frac{d_{2 m-r}(k+a)}{(k+a)^{\sigma}} \cdot \frac{k}{a}+ \\
& +\sum_{k=3}^{\infty} \frac{d_{r}(k)}{k^{\sigma}} \sum_{a=1}^{\left[\frac{k}{2}\right]} \frac{d_{2 m-r}(k-a)}{(k-a)^{\sigma}} \cdot \frac{k}{a} \ll_{m, r, \epsilon} \sum_{k=2}^{\infty} \frac{1}{k^{\sigma-\epsilon}} \sum_{a=1}^{k-1} \frac{k}{a(k+a)}+ \\
& +\sum_{k=3}^{\infty} \frac{1}{k^{\sigma-\epsilon}} \sum_{a=1}^{\left[\frac{k}{2}\right]} \frac{k}{a(k-a)} \ll \sum_{k=2}^{\infty} \frac{\log k}{k^{\sigma-\epsilon}}
\end{aligned}
$$

for some arbitrarily small $\epsilon>0$. Clearly, the last sum converges if $\sigma>1$. This completes the proof of Proposition 2.1.

We now deduce a more precise form of the residue given in Proposition 2.1. This is given in the next proposition. 
PROPOSITION 2.3. Fix $\in>0$. Let $\Re\left(s_{i}\right)>2+\epsilon, \epsilon_{i}= \pm 1,(i=1, \ldots, 2 m)$, and define $r$ to be the number of $\epsilon_{i}=1,(i=1, \ldots, 2 m)$. If $Z_{\epsilon_{1}, \ldots, \epsilon_{2 m}, k}$ denotes the multiple Dirichlet series defined in (2.7), then we have

$$
\operatorname{Res}_{w=1}\left[Z_{\epsilon_{1}, \ldots, \epsilon_{2 m}, 0}\left(s_{1}, \ldots, s_{2 m}, w\right)\right]=R_{r}\left(s_{1}, \ldots, s_{2 m}\right) . \prod_{\substack{1 \leqslant i \leqslant r \\ r+1 \leqslant j \leqslant 2 m}} \zeta\left(s_{i}+s_{j}\right),
$$

where $R_{r}\left(s_{1}, \ldots, s_{2 m}\right)$ can be holomorphically continued to the region $\Re\left(s_{i}\right)>\frac{1}{2}-\epsilon$. Further,

$$
R_{r}\left(\frac{1}{2}, \ldots, \frac{1}{2}\right)=\prod_{p}\left(1-\frac{1}{p}\right)^{m^{2}}\left(\sum_{\mu=0}^{\infty} d_{r}\left(p^{\mu}\right) d_{2 m-r}\left(p^{\mu}\right) p^{-\mu}\right),
$$

and in particular,

$$
R_{m}\left(\frac{1}{2}, \ldots, \frac{1}{2}\right)=a_{m},
$$

the constant defined in (2.5).

Proof. Define

$$
U_{r}\left(s_{1}, \ldots, s_{2 m}\right)=\sum_{\substack{\ell_{1}, \ldots, \ell_{2} m \\ \ell_{1} \cdots \ell_{r}=\ell_{r+1} \cdots \ell_{2 m}}} \ell_{1}^{-s_{1}} \cdots \ell_{2 m}^{-s_{2 m}}
$$

It follows from Proposition 2.1, that up to a permutation of the variables $s_{1}, \ldots, s_{m}$, the function $U_{r}$ is precisely the residue of $Z_{\epsilon_{1}, \ldots, \epsilon_{2}, 0}\left(s_{1}, \ldots, s_{2 m}, w\right)$ at $w=1$.

If $f(n)$ is a multiplicative function for which the sum $\sum_{n=1}^{\infty} f(n)$ converges absolutely, then we have the Euler product identity

$$
\sum_{n=1}^{\infty} f(n)=\prod_{p}\left(1+f(p)+f\left(p^{2}\right)+f\left(p^{3}\right)+\cdots\right) .
$$

It follows from (2.13) that

$$
U_{r}\left(s_{1}, \ldots, s_{2 m}\right)=\prod_{p}\left(\sum_{\mu=0}^{\infty} \sum_{\substack{e_{1}+\ldots+e_{r}=\mu \\ e_{r}+1+\cdots+e_{2}=\mu \\ e_{i} \geqslant 0,(i=1, \ldots, 2 m)}} p^{-\left(e_{1} s_{1}+\cdots+e_{2 m} s_{2 m}\right)}\right) .
$$

Let us now define

$$
R_{r}\left(s_{1}, \ldots, s_{2 m}\right)=U_{r}\left(s_{1}, \ldots, s_{2 m}\right) \cdot \prod_{\substack{1 \leqslant i \leqslant r \\ r+1 \leqslant j \leqslant 2 m}} \zeta\left(s_{i}+s_{j}\right)^{-1} .
$$

By carefully examining the Euler product for the right-hand side of (2.14), one sees that $R_{r}\left(s_{1}, \ldots, s_{2 m}\right)$ is holomorphic for $\mathfrak{R}\left(s_{i}\right)>\frac{1}{2}-\epsilon,(i=1, \ldots, 2 m)$.

Now,

$$
\sum_{\substack{e_{1}+\ldots+e_{r}=\mu \\ e_{r}+1+\cdots+e_{2}=\mu \\ e_{i} \geqslant 0,(i=1, \ldots, 2 m)}} 1=d_{r}\left(p^{\mu}\right) d_{2 m-r}\left(p^{\mu}\right) .
$$


Consequently, if we specialize the variables to $s_{1}=s_{2}=\cdots=s_{2 m}=s$, we obtain

$$
R_{r}(s, \ldots, s)=\prod_{p}\left(1-\frac{1}{p^{2 s}}\right)^{m^{2}}\left(\sum_{\mu=0}^{\infty} d_{r}\left(p^{\mu}\right) d_{2 m-r}\left(p^{\mu}\right) p^{-2 \mu s}\right) .
$$

The proof of Proposition 2.3 immediately follows upon letting $s \rightarrow \frac{1}{2}$.

\subsection{QUASI-FUNCTIONAL EQUATIONS}

Fix variables $s_{1}, s_{2}, \ldots, s_{2 m}, w$. Let $\mathcal{D} s_{1}, \ldots, s_{2 m}, w$ denote the infinite-dimensional vector space, defined over the field

$$
K_{s_{1}, \ldots, s_{2 m}}=\mathrm{C}\left((2 \pi)^{s_{1}}, \ldots,(2 \pi)^{s_{2 m}}\right),
$$

generated by the multiple Dirichlet series

$$
Z_{\epsilon_{1}, \ldots, \epsilon_{2 m}, k}\left(S_{1}, \ldots, S_{2 m}, W\right)
$$

where the variables $\epsilon_{j}, k, S_{j}$, and $W$ range over the values

$$
\begin{aligned}
& \epsilon_{j} \in\{ \pm 1\}, \quad(j=1, \ldots, 2 m) \quad k \in \mathbb{Z}, \\
& S_{j} \in\left\{s_{j}, 1-s_{j}\right\}, \quad(j=1, \ldots, 2 m), \\
& W=w+\sum_{j=1}^{2 m} \delta_{j}\left(s_{j}-\frac{1}{2}\right)
\end{aligned}
$$

with $\delta_{j} \in\{0,1\},(j=1, \ldots, 2 m)$.

For $j=1,2, \ldots, 2 m$, we will define involutions $\gamma_{j}: \mathcal{D}_{s_{1}, \ldots, s_{2 m}, w} \rightarrow \mathcal{D}_{s_{1}, \ldots, s_{2 m}, w}$.

DEFINITION 2.4. For $j=1,2, \ldots, 2 m$, we define an action $\gamma_{j}$ on

$$
Z_{\epsilon_{1}, \ldots, \epsilon_{2 m}, k}\left(S_{1}, \ldots, S_{2 m}, W\right) \in \mathcal{D}_{s_{1}, \ldots, S_{2 m}, w}
$$

(the action denoted by a right superscript) as follows:

$$
\begin{aligned}
& Z_{\epsilon_{1}, \ldots, \epsilon_{2 m}, k}\left(S_{1}, \ldots, S_{2 m}, W\right)^{\gamma_{j}} \\
& \quad=\mathrm{e}^{\frac{i \pi_{j}}{4}}(2 \pi)^{S_{j}-\frac{1}{2}} Z_{\epsilon_{1}, \ldots,-\epsilon_{j}, \ldots, \epsilon_{2 m}, k+\epsilon_{j}}\left(S_{1}, \ldots, 1-S_{j}, \ldots, S_{2 m}, W+S_{j-\frac{1}{2}}\right) .
\end{aligned}
$$

The involutions $\gamma_{j}(j=1, \ldots, 2 m)$ generate a finite Abelian group $G_{2 m}$ of $2^{2 m}$ elements which, likewise, acts on $\mathcal{D}_{s_{1}, \ldots, s_{2 m}, w}$.

We will also denote by $\gamma_{j}(j=1,2, \ldots, 2 m)$, the affine transformations induced by this action

$$
\left(s_{1}, \ldots, s_{2 m}, w\right) \stackrel{\gamma_{j}}{\longrightarrow}\left(s_{1}, \ldots, 1-s_{j}, \ldots, s_{2 m}, s_{j}+w-1 / 2\right) .
$$

By Proposition 2.1, we know that $Z_{\epsilon_{1}, \ldots, \epsilon_{2 m}, k}\left(s_{1}, \ldots, s_{2 m}, w\right)$ has holomorphic continuation to the region

$$
0<\mathfrak{R}\left(s_{i}\right)<1, \quad(i=1, \ldots, 2 m), \quad \mathfrak{R}(w)>1+m .
$$


We would like to use the functional Equation (2.1) to obtain a functional equation for the multiple Dirichlet series $Z_{\epsilon_{1}, \ldots, \epsilon_{2 m}, k}\left(s_{1}, \ldots, s_{2 m}, w\right)$. To abbreviate notation, we let $Z\left(s_{1}, \ldots, s_{2 m}, w\right)=Z_{\epsilon_{1}, \ldots, \epsilon_{2 m}, k}\left(s_{1}, \ldots, s_{2 m}, w\right)$.

We shall need an asymptotic expansion of Stirling type $[\mathrm{T}]$

$$
\begin{aligned}
& \chi(s+i t)=\mathrm{e}^{i \pi 4}\left(\frac{2 \pi}{t}\right)^{s-\frac{1}{2}}\left(\frac{2 \pi e}{t}\right)^{i t}\left\{1+\sum_{n=1}^{N} c_{n} t^{-n}+\mathrm{O}\left(t^{-N-1}\right)\right\}, \\
& \chi(s-i t)=\mathrm{e}^{i \pi 4}\left(\frac{2 \pi}{t}\right)^{s-\frac{1}{2}}\left(\frac{2 \pi e}{t}\right)^{-i t}\left\{1+\sum_{n=1}^{N} \bar{c}_{n} t^{-n}+\mathrm{O}\left(t^{-N-1}\right)\right\}
\end{aligned}
$$

(for fixed $s$ and $t \rightarrow \infty$ ),

where $c_{n}$ are certain complex constants. Such expansions are not explicitly worked out in $[\mathrm{T}]$, but they are not hard to obtain.

It now follows from Definition 2.4, Stirling's asymptotic expansion (2.16), and the functional Equation (2.1), that in the region (2.15), we have for $\gamma \in G_{2 m}$, the quasifunctional equation

$$
Z\left(s_{1}, \ldots, s_{2 m}, w\right) \sim Z\left(s_{1}, \ldots, s_{2 m}, w\right)^{\gamma}+\sum_{n=1}^{\infty} c_{n}^{\prime}(\gamma) Z\left(s_{1}, \ldots, s_{2 m}, w+n\right)^{\gamma},
$$

where $c_{n}^{\prime}\left(\gamma_{j}\right)=c_{n}$ if $\epsilon_{j}=+1$ and $c_{n}^{\prime}\left(\gamma_{j}\right)=\bar{c} n$ if $\epsilon_{j}=-1$, for $j=1,2, \ldots, 2 m$, and in general, $c_{n}^{\prime}(\gamma)$ is a linear combination of $c_{n^{\prime}}$ and $\bar{c}_{n^{\prime \prime}}$ with $n^{\prime}, n^{\prime \prime} \leqslant n$.

We shall be mainly interested in $\gamma \in G_{2 m}$ for which the action given in Definition 2.4

$$
Z_{\epsilon_{1}, \ldots, \epsilon_{2 m}, 0}\left(s_{1}, \ldots, s_{2 m}, w\right) \longrightarrow Z_{\epsilon_{1}, \ldots, \epsilon_{2 m}, 0}\left(s_{1}, \ldots, s_{2 m}, w\right)^{\gamma}
$$

stabilizes $k=0$. An element $\gamma \in G_{2 m}$ is said to stabilize $k$ relative to $\left\{\epsilon_{1}, \ldots, \epsilon_{2 m}\right\}$ provided

$$
Z_{\epsilon_{1}, \ldots, \epsilon_{2 m}, k}\left(s_{1}, \ldots, s_{2 m}, w\right)^{\gamma}=C\left(s_{1}, \ldots, s_{2 m}\right) \cdot Z_{\epsilon_{1}^{\prime}, \ldots, \epsilon_{2 m}^{\prime}, k^{\prime}}\left(s_{1}^{\prime}, \ldots, s_{2 m}^{\prime}, w^{\prime}\right)
$$

for some $C\left(s_{1}, \ldots, s_{2 m}\right) \in K_{s_{1}, \ldots, s_{2 m}}$ with $k=k^{\prime}$.

DEFINITION 2.5. Fix $\epsilon_{i}= \pm 1,(i=1, \ldots, 2 m)$. We define $G_{2 m}\left(\epsilon_{1}, \ldots, \epsilon_{2 m}\right)$ to be the subset of $G_{2 m}$ (defined in Definition 2.4) consisting of all $\gamma \in G_{2 m}$ which stabilize 0 relative to $\left\{\epsilon_{1}, \ldots, \epsilon_{2 m}\right\}$.

PROPOSITION 2.6. Let $1 \leqslant r \leqslant 2 m$, and

$$
\epsilon_{i_{1}}=\epsilon_{i_{2}}=\cdots=\epsilon_{i r}=+1, \quad \epsilon_{i_{r+1}}=\epsilon_{i_{r+2}}=\cdots=\epsilon_{i_{2 m}}=-1 .
$$

Then $G_{2 m}\left(\epsilon_{1}, \ldots, \epsilon_{2 m}\right)$ is the subgroup of $G_{2 m}$ which is generated by the elements $\gamma_{i_{\mu}} \cdot \gamma_{i_{v}}$ with $1 \leqslant \mu \leqslant r, r+1 \leqslant v \leqslant 2 m$.

Proof. Note that if we write $\gamma=\gamma_{i} \cdot \gamma_{j}$ (with $i \neq j$ ) then under the action (2.18) we see that 


$$
\{k=0\} \stackrel{\gamma}{\longrightarrow}\left\{k=\epsilon_{i}+\epsilon_{j}\right\} .
$$

So if we choose $i$ from the set $\left\{i_{1}, \ldots, i_{r}\right\}$ and $j$ from the set $\left\{i_{r+1}, \ldots, i_{2 m}\right\}$ then we see that $\{k=0\}$ is stabilized. It easily follows that these elements generate a group and every element of this group stabilizes 0 relative to $\left\{\epsilon_{1}, \ldots, \epsilon_{2 m}\right\}$. Furthermore, every element which stabilizes 0 relative to $\left\{\epsilon_{1}, \ldots, \epsilon_{2 m}\right\}$ must lie in this group.

Remark. We introduced the group $G_{2 m}\left(\epsilon_{1}, \ldots, \epsilon_{2 m}\right)$ because it is precisely this group which gives the reflections of the polar divisor at $w=1$ of the multiple Dirichlet series $Z_{\epsilon_{1}, \ldots, \epsilon_{2 m}, 0}\left(s_{1}, \ldots, s_{2 m}, w\right)$. This will be further explained in the next section.

\subsection{A FUNDAMENTAL CONJECTURE FOR THE RIEMANN ZETA FUNCTION}

We observed in Proposition 2.1 that the hyperplane $w-1=0$ belongs to the polar divisor of the multiple Dirichlet series $Z_{\epsilon_{1}, \ldots, \epsilon_{2 m}, k}$ if and only if $k=0$. It was also seen that this hyperplane is the only possible pole in the region $\mathcal{F}$ defined by

$$
\begin{gathered}
\mathcal{F}=\left\{\left(s_{1}, \ldots, s_{2 m}, w\right) \in \mathbb{C}^{2 m+1} \mid \Re\left(s_{i}\right)>0(i=1, \ldots, 2 m), \Re(w)>1+m\right\} \cup \\
\cup\left\{\left(s_{1}, \ldots, s_{2 m}, w\right) \in \mathbb{C}^{2 m+1} \mid \Re(w)>0, \Re\left(s_{i}\right)>2(i=1, \ldots, 2 m)\right\} .
\end{gathered}
$$

Now, the set $\bigcap_{\gamma \in G_{2 m}} \gamma(\mathcal{F})$ is nonempty, since it contains points for which $\Re\left(s_{i}\right) \sim 1 / 2(i=1, \ldots, 2 m)$ and $\mathfrak{R}(w)$ is sufficiently large. It follows from the quasifunctional Equation (2.17) that the multiple Dirichlet series $Z_{\epsilon_{1}, \ldots, \epsilon_{2 m}, 0}$ have meromorphic continuation to the convex closure of the region $\bigcup_{\gamma \in G_{2 m}} \gamma(\mathcal{F})$ with poles, precisely, at the reflections of the hyperplane $w-1=0$ under $G_{2 m}\left(\epsilon_{1}, \ldots, \epsilon_{2 m}\right)$. In order to obtain the continuation, it is understood that we first multiply $Z_{\epsilon_{1}, \ldots, \epsilon_{2 m}, 0}$ by certain linear factors in order to cancel its poles. We propose the following conjecture.

CONJECTURE 2.7. The functions $Z_{\epsilon_{1}, \ldots, \epsilon_{2 m}, 0}$ have meromorphic continuation to a tube domain in $\mathrm{C}^{2 m+1}$ which contains the point $\left(\frac{1}{2}, \ldots, \frac{1}{2}, 1\right)$. All these functions have the same polar divisor passing through this point consisting of all the reflections of the hyperplane $w-1=0$ under the group $G_{2 m}\left(\epsilon_{1}, \ldots, \epsilon_{2 m}\right)$. Moreover, the functions

$$
Z_{\epsilon_{1}, \ldots, \epsilon_{2}, 0}\left(\frac{1}{2}, \ldots, \frac{1}{2}, w\right)
$$

are holomorphic for $\Re(w)>1$.

THEOREM 2.8. Conjecture 2.7 implies the Keating-Snaith-Conrey-Farmer Conjecture (2.3).

Proof. From now on, we fix

$$
\epsilon_{1}=\epsilon_{2}=\cdots=\epsilon_{m}=+1, \quad \epsilon_{m+1}=\epsilon_{m+2}=\cdots=\epsilon_{2 m}=-1,
$$


and let $G_{2 m}^{\prime}$ denote the group $G_{2 m}\left(\epsilon_{1}, \ldots, \epsilon_{2 m}\right)$. The reflections of the hyperplane $w-1=0$ under the group $G_{2 m}^{\prime}$ are given by

$$
\delta_{1} s_{1}+\cdots+\delta_{2 m} s_{2 m}+w-\frac{\delta_{1}+\cdots+\delta_{2 m}+2}{2}=0,
$$

where $\delta_{i}=0$ or 1 and $\delta_{1}+\cdots+\delta_{m}=\delta_{m+1}+\cdots+\delta_{2 m}$.

In this and the next section we require a version of the Wiener-Ikehara Tauberian theorem. Stark has proved a vast generalization of this theorem, [St]. We will quote here a limited a case of his result which is sufficient for our needs.

TAUBERIAN THEOREM (Stark). Let $S(x)$ be a nondecreasing function of $x$ and let

$$
Z(w)=\int_{1}^{\infty} S(t) \cdot t^{-w} \frac{\mathrm{d} t}{t} .
$$

Let $P(w)=\gamma_{M}+\gamma_{M-1}(w-1)+\cdots+\gamma_{0}(w-1)^{M},(M \geqslant 0)$ be a polynomial with $\gamma_{M} \neq 0$ such that $Z(w)-P(w)(w-1)^{-M-1}$ is holomorphic for $\Re(w)>1$ and continuous for $\Re(w)=1$. Then

$$
S(x) \sim \frac{\gamma_{M}}{M !} \cdot x(\log x)^{M}, \quad(\text { as } x \rightarrow \infty) .
$$

We now let $z(t)=\zeta(1 / 2+i t)^{m}$ and $S(x)=\int_{0}^{x}|z(t)|^{2} \mathrm{~d} t$ in the Tauberian theorem. It follows by integration by parts that

$$
\int_{1}^{\infty} S(t) \cdot t^{-w} \frac{\mathrm{d} t}{t}=\frac{1}{w} \int_{1}^{\infty}|z(t)| 2 t^{-w} \mathrm{~d} t .
$$

Consequently, it is enough to show that

$$
\lim _{w \rightarrow 1}(w-1)^{m^{2}+1} Z_{\epsilon_{1}, \ldots, \epsilon_{2 m}, 0}\left(s_{1}, \ldots, s_{2 m}, w\right)=g_{2 m} a_{2 m} m^{2} !,
$$

where

$$
g_{2 m}=\prod_{\ell=0}^{m-1}=\frac{\ell !}{(\ell+m) !},
$$

and $a_{2 m}$ is the constant given in (2.5).

Let $U\left(s_{1}, \ldots, s_{2 m}, w\right)$ denote the function defned by

$$
\frac{1}{w-1} R_{m}\left(s_{1}, \ldots, s_{2 m}\right) \prod_{i=1}^{m} \prod_{j=m+1}^{2 m} \zeta\left(s_{i}+s_{j}\right) .
$$

Then Conjecture 2.7 implies that

$$
Z_{\epsilon_{1}, \ldots, \epsilon_{2 m}, 0}\left(s_{1}, \ldots, s_{2 m}, w\right)-\sum_{\gamma \in G_{2 m}^{\prime}} U\left(\gamma\left(s_{1}, \ldots, s_{2 m}, w\right)\right)
$$

is holomorphic around $\left(\frac{1}{2}, \ldots, \frac{1}{2}, 1\right)$. The proof of Theorem 2.8 is an immediate consequence of the following proposition. 
PROPOSITION 2.9. For $m=1,2, \ldots$, let $G_{2 m}^{\prime}$ denote the subgroup of $G_{2 m}^{\prime}$ generated by the involutions $\gamma_{i j}=\gamma_{i} \cdot \gamma_{j},(i=1, \ldots, m$ and $j=m+1, \ldots, 2 m)$. Then we have

$$
\lim _{w \rightarrow 1} \lim _{\left(s_{1}, \ldots, s_{2 m}\right) \rightarrow\left(\frac{1}{2}, \ldots, \frac{1}{2}\right)}\left[(w-1)^{m^{2}+1} \sum_{\gamma \in G_{2 m}^{\prime}} U\left(\gamma\left(s_{1}, \ldots, s_{2 m}, w\right)\right)\right]=a_{2 m} g_{2 m} m^{2} !
$$

where

$$
g_{2 m}=\prod_{\ell=0}^{m-1} \frac{\ell !}{(\ell+m) !},
$$

and $a_{2 m}$ is the constant given in (2.5).

Proof. We start by taking the Taylor expansion of

$$
U\left(s_{1}, \ldots, s_{2 m}, w\right)=a_{2 m} \frac{f^{*}\left(s_{1}, \ldots, s_{2 m}\right)}{(w-1) \prod_{i=1}^{m} \prod_{j=m+1}^{2 m}\left(s_{i}+s_{j}-1\right)}
$$

around $\left(s_{1}, \ldots, s_{2 m}\right)=\left(\frac{1}{2}, \ldots, \frac{1}{2}\right)$. Here

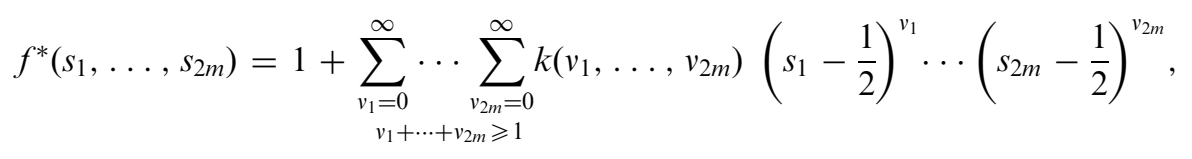

(with $k\left(v_{1}, \ldots, v_{2 m}\right) \in \mathrm{C}$ ), will be a holomorphic function which is symmetric separately with respect to the variables $s_{1}, \ldots, s_{m}$ and $s_{m+1}, \ldots, s_{2 m}$.

Now, make the change of variables $s_{i}=\frac{1}{2}+u_{i}$ for $i=1,2, \ldots, 2 m$, and $w=v+1$. Then, for $i=1, \ldots, m$ and $j=m+1, \ldots, 2 m$, the involutions $\gamma_{i j}$ are transformed to

$$
\begin{aligned}
& \left(u_{1}, \ldots, u_{i}, \ldots, u_{m}, \ldots, u_{j}, \ldots, u_{2 m}, v\right) \stackrel{\gamma_{i j}}{\longrightarrow} \\
& \quad\left(u_{1}, \ldots,-u_{j}, \ldots, u_{m}, \ldots,-u_{i}, \ldots, u_{2 m}, u_{i}+u_{j}+v\right) .
\end{aligned}
$$

Henceforth, we denote by $G_{2 m}^{\prime}$ the group generated by the above involutions.

Then by (2.22), it is enough to prove that

$$
\lim _{v \mapsto 0} \lim _{v\left(u_{1}, \ldots, u_{2 m}\right) \rightarrow(0, \ldots, 0)}\left[v^{m^{2}+1} \sum_{\gamma \in G_{2 m}^{\prime}} H_{f}\left(\gamma\left(u_{1}, \ldots, u_{2 m}, v\right)\right)\right]=g_{2 m} m^{2} !,
$$

where

$$
H_{f}\left(u_{1}, \ldots, u_{2 m}, v\right)=\frac{1}{v} \cdot \frac{f\left(u_{1}, \ldots, u_{2 m}\right)}{\prod_{i=1}^{m} \prod_{j=m+1}^{2 m}\left(u_{i}+u_{j}\right)},
$$

and $f$ (which is simply related to $f^{*}$ ) is a certain holomorphic function and symmetric separately with respect to the variables $u_{1}, \ldots, u_{m}$ and $u_{m+1}, \ldots, u_{2 m}$. It also satisfies $f(0, \ldots, 0)=1$. 
The proof of the Proposition is an immediate consequence of the following Lemma.

LEMMA 2.10. The limit (2.23) exists.

Proof. Let $f=\sum_{k \geqslant 0} f_{k}$, where $f_{k}$ (for $k=0,1,2, \ldots$ ) is a homogeneous polynomial of degree $k$ and which is also symmetric separately with respect to the variables $u_{1}, \ldots, u_{m}$ and $u_{m+1}, \ldots, u_{2 m}$. Here $f_{0}=1$. It follows that $H_{f}=\sum_{k \geqslant 0} H_{f_{k}}$. Since the action of the group $G_{2 m}^{\prime}$ commutes with permutations of the variables $u_{1}, \ldots, u_{2 m}$, it easily follows that

$$
\sum_{\gamma \in G_{2 m}^{\prime}} H_{f_{k}}\left(\gamma\left(u_{1}, \ldots, u_{2 m}, v\right)\right)
$$

is also symmetric separately with respect to the variables $u_{1}, \ldots, u_{m}$ and $u_{m+1}, \ldots, u_{2 m}$.

Define

$$
\begin{aligned}
N_{f_{k}}\left(u_{1}, \ldots, u_{2 m}, v\right)= & {\left[\prod_{\substack{\delta_{1}=0 \\
\delta_{1}+\cdots+\delta_{m}=\delta_{m+1}+\cdots+\delta_{2 m}}}^{1} \cdots \prod_{d_{2 m}=0}^{1}\left(v+\delta_{1} u_{1}+\cdots+\delta_{2 m} u_{2 m}\right)\right] \times } \\
& \times \sum_{\gamma \in G_{2 m}^{\prime}} H_{f_{k}}\left(\gamma\left(u_{1}, \ldots, u_{2 m}, v\right)\right) .
\end{aligned}
$$

Then $N_{f_{k}}$ is invariant under the group $G_{2 m}^{\prime}$, and it is symmetric separately in the variables $u_{1}, \ldots, u_{m}$, and $u_{m+1}, \ldots, u_{2 m}$. Moreover, by checking the action of the group $G_{2 m}^{\prime}$ on the product

$$
\prod_{i=1}^{m} \prod_{j=m+1}^{2 m}\left(u_{i}+u_{j}\right)
$$

it follows that $N_{f_{k}}$ is a rational function

$$
N_{f_{k}}=\frac{N_{f_{k}}^{*}}{D_{f_{k}}^{*}}
$$

with denominator

$$
D_{f_{k}}^{*}\left(u_{1}, \ldots, u_{2 m}, v\right)=\prod_{i=1}^{m} \prod_{j=m+1}^{2 m}\left(u_{i}+u_{j}\right) \prod_{1 \leqslant i<j \leqslant m}\left(u_{i}-u_{j}\right) \prod_{m+1 \leqslant i><j \leqslant 2 m}\left(u_{i}-u_{j}\right) .
$$

The function $N_{f_{k}}$ is, in fact, a polynomial in the variables $u_{1}, \ldots, u_{2 m}, v$. To see this, we first observe that, for $1 \leqslant i<j \leqslant m$ or $m+1 \leqslant i<j \leqslant 2 m$,

$$
N_{f_{k}}^{*}\left(\ldots, u_{i}, \ldots, u_{j}, \ldots, v\right)=-N_{f_{k}}^{*}\left(\ldots, u_{j}, \ldots, u_{i}, \ldots, v\right) .
$$

This implies that

$$
N_{f_{k}}^{*}\left(\ldots, u_{i}, \ldots, u_{i}, \ldots, v\right)=0
$$


which gives

$$
\left(u_{i}-u_{j}\right) \mid N_{f_{k}}^{*}\left(u_{1}, \ldots, u_{2 m}, v\right)
$$

for $1 \leqslant i<j \leqslant m$ or $m+1 \leqslant i<j \leqslant 2 m$. On the other hand, it can be observed that

$$
D_{f_{k}}^{*}\left(u_{1}, \ldots, u_{2 m}, v\right)=-D_{f_{k}}^{*}\left(\gamma_{i j}\left(u_{1}, \ldots, u_{2 m}, v\right)\right) \text {, }
$$

for $i=1, \ldots, m$ and $j=m+1, \ldots, 2 m$. Since the function $N_{f_{k}}$ is invariant under the group $G_{2 m}^{\prime}$, it follows from (2.24), and (2.28) that

$$
N_{f_{k}}^{*}\left(u_{1}, \ldots, u_{2 m}, v\right)=-N_{f_{k}}^{*}\left(\gamma_{i j}\left(u_{1}, \ldots, u_{2 m}, v\right)\right) \text {, }
$$

for $1 \leqslant i<j \leqslant m$ or $m+1 \leqslant i<j \leqslant 2 m$. This together with (2.27) implies that

$$
\left(u_{i}+u_{j}\right) \mid N_{f_{k}}^{*}\left(u_{1}, \ldots, u_{2 m}, v\right)
$$

for $1 \leqslant i \leqslant m$ and $m+1 \leqslant j=2 m$. Finally, it follows from (2.27) and (2.30) that for $\Re(v)>0$, the limit

$$
\lim _{\left(u_{1}, \ldots, u_{2 m}\right) \mapsto(0, \ldots, 0)} \sum_{\gamma \in G_{2 m}^{\prime}} H_{f_{k}}\left(\gamma\left(u_{1}, \ldots, u_{2 m}, v\right)\right)
$$

exists. Our lemma is proved.

Now, set $u_{i}=u_{m+i}=i \cdot \epsilon($ for $i=1,2, \ldots, m-1), u_{m}=0$ and $u_{2 m}=m \cdot \epsilon$. By induction over $m$, it can be checked that

$$
\left\{\delta_{1} u_{1}+\cdots+\delta_{2 m} u_{2 m} \mid \delta_{i}=0,1 ; \delta_{1}+\cdots+\delta_{m}=\delta_{m+1}+\cdots+\delta_{2 m}=\left\{0,1, \ldots, m^{2}\right\} .\right.
$$

From Lemma 2.10 and (2.31), it follows that for $k=0,1,2, \ldots$,

$$
\sum_{\gamma \in G_{2 m}^{\prime}} H_{f_{k}}\left(\gamma\left(u_{1}, \ldots, u_{2 m}, v\right)\right)=\frac{P k(\epsilon, v)}{\prod_{\ell=0}^{m^{2}}(v+\ell \epsilon)},
$$

where $P_{k}(\epsilon, v)$ is a homogeneous polynomial of degree $k$ in the two variables $\epsilon, v$.

Consequently

$$
\lim _{v \mapsto 0} \lim _{\epsilon \mapsto 0} v^{m^{2}+1} \sum_{\gamma \in G_{2 m}^{\prime}} H_{f_{k}}\left(\gamma\left(u_{1}, \ldots, u_{2 m}, v\right)\right)=0
$$

if $k>0$, and the limit exists if $k=0$. Using that $f_{0}=1$, the proposition follows by taking the residue at $v=0$ on both sides of (2.32).

\section{Moments of Quadratic Dirichlet $L$-Functions}

Let

$$
\chi_{d}(n)=\left\{\begin{array}{lll}
\left(\frac{d}{n}\right) & \text { if } & d \equiv 1(\bmod 4), \\
\left(\frac{4 d}{n}\right) & \text { if } & d \equiv 2,3(\bmod 4),
\end{array}\right.
$$


denote Kronecker's symbol which is precisely the Dirichlet character associated to the quadratic field $\mathrm{Q}(\sqrt{d})$. For $\mathbb{R}(s)>1$ we define

$$
L\left(s, \chi_{d}\right)=\sum_{n=1}^{\infty} \frac{\chi_{d}(n)}{n^{3}},
$$

to be the classical Dirichlet $L$-function associated to $\chi_{d}$.

We shall always denote by $\sum_{|d|}$ a sum ranging over fundamental discriminants of quadratic fields. We shall consider moments as $x \rightarrow \infty$. Jutila [J] was the First to obtain the moments

$$
\sum_{|d| \leqslant x} L\left(\frac{1}{2}, \chi_{d}\right) \sim a_{1} \frac{6}{\pi^{2}} x \log \left(x^{\frac{1}{2}}\right)
$$

and

$$
\sum_{|d| \leqslant x} L\left(\frac{1}{2}, \chi_{d}\right)^{2} \sim 2 \cdot \frac{a_{2}}{3 !} \frac{6}{\pi^{2}} x \log ^{3}\left(x^{\frac{1}{2}}\right)
$$

with

$$
a_{m}=\prod_{p} \frac{\left(1-\frac{1}{p}\right)^{\frac{m(m+1)}{2}}}{\left(1+\frac{1}{p}\right)}\left(\frac{\left(1-\frac{1}{\sqrt{P}}\right)^{-m}+\left(1+\frac{1}{\sqrt{P}}\right)^{-m}}{2}+\frac{1}{p}\right), \quad(m=1,2, \ldots) .
$$

Subsequently, Soundararajan [So] showed that

$$
\sum_{|d| \leqslant x} L\left(\frac{1}{2}, \chi_{d}\right)^{3} \sim 16 \cdot \frac{a 3}{6 !} \frac{6}{\pi^{2}} x \log ^{6}\left(x^{\frac{1}{2}}\right) .
$$

He also conjectured that

$$
\sum_{|d| \leqslant x} L\left(\frac{1}{2}, \chi_{d}\right)^{4} \sim 768 \cdot \frac{a_{4}}{10 !} \frac{6}{\pi^{2}} x \log ^{10}\left(x^{\frac{1}{2}}\right) .
$$

Motivated by the fundamental work of Katz and Sarnak [K-S], who introduced symmetry types associated to families of $L$-functions, the previous results (3.1), (3.2), (3.4), (3.5), and calculations of Keating and Snaith [Ke-Sn-2] based on random matrix theory, Conrey and Farmer have made the following conjecture.

CONJECTURE 3.1. For every positive integer $m$, and $x \rightarrow \infty$,

$$
\sum_{|d| \leqslant x} L\left(\frac{1}{2}, \chi_{d}\right)^{m} \sim \frac{6}{\pi^{2}} a_{m} \cdot \prod_{\ell=1}^{m} \frac{\ell !}{(2 \ell) !} \cdot x(\log x)^{M},
$$

where $M=\frac{m(m+1)}{2}$. 


\subsection{THE MULTIPLE DIRICHLET SERIES FOR THE FAMILY OF QUADRATIC $L$-FUNCTIONS}

For $w, s_{1}, s_{2}, \ldots, s_{m} \in \mathbb{C}$ with $\Re(w)>1$ and $\Re\left(s_{i}\right)>1(i=1,2, \ldots, m)$, consider the absolutely convergent multiple Dirichlet series

$$
Z\left(s_{1}, s_{2}, \ldots, s_{m}, w\right)=\sum_{d} \frac{L\left(s_{1}, \chi_{d}\right) \cdot L\left(s_{2}, \chi_{d}\right) \cdots L\left(s_{m}, \chi_{d}\right)}{|d|^{w}}
$$

where the sum ranges over fundamental discriminants of quadratic fields.

Recently, (see [B-F-H-1]), for the special cases $m=1,2,3$ a new proof of Conjecture 3.1, based on the meromorphic continuation of $Z\left(s_{1}, \ldots, s_{m}, w\right)$, was obtained. Unfortunately, the method of proof breaks down when $m \geqslant 4$ because there are not enough functional equations of $Z\left(s_{1}, \ldots, s_{m}, w\right)$ to obtain its meromorphic continuation slightly beyond the first significant polar divisor at $w=1$, and, $s_{1} \rightarrow \frac{1}{2}$, $s_{2} \rightarrow \frac{1}{2}, \ldots, s_{m} \rightarrow \frac{1}{2}$.

We shall show that $Z\left(s_{1}, \ldots, s_{m}, w\right)$ (suitably modified by breaking it into two parts and multiplying by appropriate gamma factors) satisfies the functional equations

$$
\left(s_{1}, \ldots, s_{m}, w\right) \stackrel{\alpha_{i}}{\rightarrow}\left(s_{1}, \ldots, 1-s_{i}, \ldots, s_{m}, w+s_{i}-\frac{1}{2}\right), \quad(i=1,2, \ldots, m) .
$$

We then show that for $\Re\left(s_{i}\right)$ sufficiently large $(i=1,2, \ldots, m)$, that $Z\left(s_{1}, \ldots, s_{m}, w\right)$ has a simple pole at $w=1$, and that the residue has analytic continuation to the region

$$
\mathfrak{R}\left(s_{i}\right)>\frac{1}{2}-\epsilon, \quad(i=1,2, \ldots, m),
$$

for any fixed $\epsilon>0$. The residue of $Z\left(s_{1}, \ldots, s_{m}, w\right)$ at $w=1$ and $s_{1} \rightarrow \frac{1}{2}, \ldots, s_{m} \rightarrow \frac{1}{2}$ can be computed exactly and coincides with the constant in Conjecture 3.1. This is the basis for Conjecture 3.6 given in Section 3.2.

In order to determine the residues and poles of $Z\left(s_{1}, \ldots, s_{m}, w\right)$, it is necessary to introduce a modifed multiple Dirichlet series defined by

$$
Z_{v}^{ \pm}\left(s_{1}, \ldots, s_{m}, w\right)=\sum_{\substack{ \pm d>0 \\ d \equiv v(m o d 4) \\ d s q \text { free }}} \frac{L\left(s_{1}, \chi_{d}\right) \cdots L\left(s_{m}, \chi_{d}\right)}{|d|^{w}} .
$$

We set

$$
\begin{aligned}
Z^{ \pm}\left(s_{1}, \ldots, s_{m}, w\right)= & Z_{1}^{ \pm}\left(s_{1}, \ldots, s_{m}, w\right)+ \\
& +4^{-w}\left(Z_{2}^{ \pm}\left(s_{1}, \ldots, s_{m}, w\right)+Z_{3}^{ \pm}\left(s_{1}, \ldots, s_{m}, w\right)\right) .
\end{aligned}
$$

Further, we define

$$
\widehat{Z^{+}}\left(s_{1}, \ldots, s_{m}, w\right)=\left(\prod_{i=1}^{m} \pi^{-\frac{s_{i}}{2}} \Gamma\left(\frac{s_{i}}{2}\right)\right) \cdot Z^{+}\left(s_{i} \ldots, s_{m}, w\right)
$$


and

$$
\widehat{Z^{-}}\left(s_{1}, \ldots, s_{m}, w\right)=\left(\prod_{i=1}^{m} \pi^{-\frac{s_{i}+1}{2}} \Gamma\left(\frac{s_{i}+1}{2}\right)\right) \cdot Z^{-}\left(s_{1}, \ldots, s_{m}, w\right) .
$$

The following two propositions summarize the analytic properties of the functions $Z^{ \pm}$.

PROPOSITION 3.2. For $\sigma>0$, the functions $Z^{ \pm}$can be meromorphically continued to the domain

$$
\Re\left(s_{i}\right)>-\sigma \quad(i=1,2, \ldots, m), \quad \Re(w)>1+m \cdot\left(\frac{1}{2}+\sigma\right) .
$$

The only poles in this region are at $s_{i}=1,(i=1, \ldots, m)$. Moreover, both $\widehat{Z^{ \pm}}$are invari ant under the finite abelian group $G_{m}$ (of $2^{m}$ elements) generated by the involutions

$$
\left(s_{1}, \ldots, s_{m}, w\right) \stackrel{\alpha_{i}}{\longrightarrow}\left(s_{1}, \ldots, 1-s_{i}, \ldots, s_{m}, w+s_{i}-\frac{1}{2}\right), \quad(i=1,2, \ldots, m) .
$$

Proof. Note that the term corresponding to $d=1$ in the definition of $Z^{ \pm}$as a Dirichlet series (see (3.8), (3.9)) contributes $\zeta\left(s_{1}\right) \cdots \zeta\left(s_{m}\right)$ which has poles at $s_{i}=1$ for $i=1, \ldots, m$. The functional equation of $L\left(s, \chi_{d}\right)$ (see [D])may be written in the form

$$
\begin{aligned}
\Lambda\left(s, \chi_{d}\right) & =\pi^{-\frac{s+a}{2}} \Gamma\left(\frac{s+a}{2}\right) L\left(s, \chi_{d}\right) \\
& =|D|^{\frac{1}{2}-s} \Lambda\left(1-s, \chi_{d}\right),
\end{aligned}
$$

where $a=0,1$ is chosen so that $\chi_{d}(-1)=(-1)^{a}$, and

$$
D=\left\{\begin{array}{lll}
d & \text { if } & d \equiv 1(\bmod 4) \\
4 d & \text { if } & d \equiv 2,3(\bmod 4)
\end{array}\right.
$$

is the conductor of $\chi_{d}$. It follows from (3.12) that for $\mathfrak{R}(s)>-\sigma$, and $d>1$,

$$
\left|L\left(s, \chi_{d}\right)\right|=\mathrm{O}\left(|d|^{\frac{1}{2}+\sigma}\right),
$$

where the $\mathrm{O}$ constant depends at most on $\mathfrak{\Im}(s)$. Plugging the estimate (3.13) into the Definition (3.8) of $Z_{v}^{ \pm}\left(s_{1}, s_{2}, \ldots, s_{m}, w\right)$ (with $\left.v=1,2,3\right)$ viewed as an infinite series, we see that the series (with terms $d>1$ ) converges absolutely provided $\Re(w) \geq 1+m \cdot\left(\frac{1}{2}+\sigma\right)$. This establishes the first part of Proposition 3.2.

Now, both $\widehat{Z^{ \pm}}$are invariant under permutations of the variables $s_{1}, s_{2}, \ldots, s_{m}$. Therefore, to prove the invariance under the group $G_{m}$, it suffices to show the invariance under the transformation $\alpha_{1}$, say. To show this invariance, we invoke the functional equation (3.12) with $s=s_{1}$. The invariance under the transformation $\alpha_{1}$ immediately follows. 
PROPOSITION 3.3. The functions $\zeta(2 w) Z^{ \pm}$can be meromorphically continued for $\Re(w)>0$ and $\Re\left(s_{i}\right)$ sufficiently large $(i=1,2, \ldots, m)$. They are holomorphic in this region except for a simple pole at $w=1$ with residue

$$
\begin{aligned}
\operatorname{Res}_{w=1}\left[\zeta(2 w) Z^{+}\left(s_{1}, \ldots, s_{m}, w\right)\right] & =\operatorname{Res}_{w=1}\left[\zeta(2 w) Z^{-}\left(s_{1}, \ldots, s_{m}, w\right)\right] \\
& =\frac{1}{2} \sum_{\substack{n_{1} \ldots, n_{m} \\
n_{1} \cdots n_{m}=\square}} \frac{\prod_{p \mid n_{1} \cdots n_{m}}\left(1+p^{-1}\right)^{-1}}{n_{1}^{s_{1}} \cdots n_{m}^{s_{m}}} .
\end{aligned}
$$

Here $\square$ denotes any square integer, and the sum ranges over all m-tuples $\left\{n_{1}, \ldots, n_{m}\right\}$ of positive integers.

Proof. It follows from (3.8) that

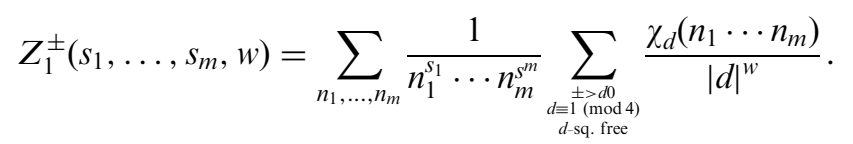

For any fixed $m$-tuple $\left\{n_{1}, \ldots, n_{m}\right\}$ of positive integers, we may write $n_{1} \cdots n_{m}=2^{c} n N^{2} M^{2}$ so that

- $n$ is square-free

- $p|N \Rightarrow p| n$

- $n$ and $M$ are both odd and coprime.

It immediately follows from (3.15) that the inner sum in (3.14) can be rewritten as

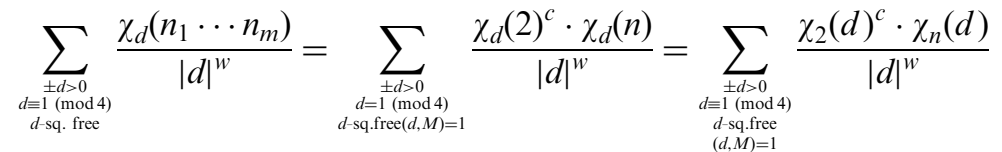

$$
\begin{aligned}
& =\frac{1}{2} \sum_{\substack{ \pm d>0 \\
\text { dsd.free } \\
(d, 2 M)=1}} \frac{\chi_{2}(d)^{c} \cdot \chi_{n}(d)}{|d|^{w}}+\frac{1}{2} \sum_{\substack{d d>0 \\
\text { dsd.firee } \\
(d, 2, M)=1}} \frac{\chi_{2}(d)^{c} \cdot \chi_{-1}(d) \cdot \chi_{n}(d)}{|d|^{w}} .
\end{aligned}
$$

Here we have used the law of quadratic reciprocity

$$
\chi_{d}(2)=\left\{\begin{array}{lll}
\chi_{2}(d)=(-1)^{\frac{d^{2}-1}{8}} & \text { if } \quad d \equiv 1(\bmod 4) \\
0 & \text { if } \quad d \equiv 2,3(\bmod 4)
\end{array}\right.
$$

and

$$
\chi_{d}(n)=\chi_{n}(d) \cdot(-1)^{\frac{(d-1)(n-1)}{4}}, \quad(d, n, \text { odd }) .
$$

Further, for $d$ odd, $\frac{1}{2}\left(1+\chi_{-1}(d)\right)$ is 1 or 0 according as $d \equiv 1$ or $3(\bmod 4)$. This last assertion follows from the identity 


$$
\chi_{-1}(d)=\left(\frac{-4}{d}\right)= \begin{cases}(-1)^{\frac{|d|-1}{2}}+\frac{\operatorname{sgn}(d)-1}{2} & \text { if } d \equiv 1(\bmod 2) \\ 0 & \text { if } d \equiv 0(\bmod 2)\end{cases}
$$

In order to complete the proof of Proposition 3.3 we require the following lemma.

LEMMA 3.4. Let $\chi$ be a primitive quadratic Dirichlet character of conductor $n$, and let $b$ be any positive integer. If $L_{b}(w, \chi)$ is the function defined by

$$
L_{b}(w, \xi)=\sum_{\substack{d>0 \\ d \text {-sq.free } \\(d, b)=1}} \frac{\chi(d)}{d^{w}}
$$

then $\zeta(2 w) L_{b}(w, \chi)$ can be meromorphically continued to $\Re(w)>0$. It is analytic everywhere in this region, unless $n=1$ (i.e., $L(w, \chi)=\zeta(w)$ ), when it has exactly one simple pole at $w=1$ with residue

$$
\operatorname{Res}_{w=1}\left[\zeta(2 w) L_{b}(w, \chi)\right]=\prod_{p \mid b}\left(1+\frac{1}{p}\right)^{-1} .
$$

Proof. The proof of Lemma 3.4 is a simple consequence of the elementary identity

$$
L_{b}(w, \chi)=\frac{L(w, \chi)}{\zeta(2 w)} \cdot \prod_{p \mid b}\left(1+\chi(p) p^{-w}\right)^{-1} \prod_{p \mid n}\left(1-p^{2 w}\right)^{-1}
$$

It immediately follows from (3.16) and Lemma 3.4 that

$$
\sum_{\substack{ \pm d>0 \\ d \equiv 1(\bmod 4) \\ d \text {-sq.free }}} \frac{\chi_{d}\left(n_{1} \cdots n_{m}\right)}{|d|^{w}}=\frac{1}{2} L_{2 M}\left(w, \chi_{2}^{c} \cdot \chi_{n}\right)+\frac{1}{2} L_{2 M}\left(w, \chi_{2}^{c} \cdot \chi_{-1} \cdot \chi_{n}\right)
$$

and that the right-hand side of (3.17) has a meromorphic continuation to $\Re_{(w)}>0$. Moreover, it is holomorphic in this region unless $n=1$ and $c \equiv 0(\bmod 2)$, in which case there is exactly one simple pole at $w=1$ with residue

$$
\operatorname{Res}_{w=1}\left[\zeta(2 w) \cdot \sum_{\substack{ \pm d>0 \\ d \equiv 1(\bmod 4) \\ d \text {-sq.free }}} \frac{\chi_{d}\left(n_{1} \cdots n_{m}\right)}{|d|^{w}}\right]=\frac{1}{2} \prod_{p \mid 2 M}\left(1+\frac{1}{p}\right)^{-1} .
$$

Now, if we sum both sides of (3.18) over all $m$-tuples $\left\{m_{1}, \ldots, m_{n}\right\}$, it is clear that there will only be a contribution to the residue coming from $m$-tuples where $m_{1} \cdots m_{n}=\square$. Combining equations (3.14) and (3.18), and then removing the factor $1+2^{-1}$ when $n_{1} \cdots n_{m}$ is odd gives 


$$
\begin{aligned}
\operatorname{Res}_{w=1} & {\left[\zeta(2 w) \cdot Z_{1}^{ \pm}\left(s_{1}, \ldots, s_{m}, w\right)\right] } \\
& =\frac{1}{2} \sum_{\substack{n_{1} \ldots n_{m} \\
n_{1} \cdots n_{m}=\square}} \frac{\prod_{p \mid 2 n_{1} \cdots n_{m}}\left(1+p^{-1}\right)^{-1}}{n_{1}^{s_{1}} \cdots n_{m}^{s_{m}}} \\
& =\frac{1}{2} \sum_{\substack{2 \mid n_{1} \cdots n_{m} \\
n_{1} \cdots n_{m}=\square}} \frac{\prod_{p \mid n_{1} \cdots n_{m}}\left(1+p^{-1}\right)^{-1}}{n_{1}^{s_{1}} \cdots n_{m}^{s_{m}}}+\frac{1}{3} \sum_{\substack{2 \mid n_{1} \cdots n_{m} \\
n_{1} \cdots n_{m}=\square}} \frac{\prod_{p \mid n_{1} \cdots n_{m}}\left(1+p^{-1}\right)^{-1}}{n_{1}^{s_{1}} \cdots n_{m}^{s_{m}}} .
\end{aligned}
$$

In a completely analogous manner, we can also obtain

$$
\operatorname{Res}_{w=1}\left[\zeta(2 w) \cdot Z_{v}^{ \pm}\left(s_{1}, \ldots, s_{m}, w\right)\right]=\frac{1}{3} \sum_{\substack{\mid n_{1} \cdots n_{m} \\ n_{1} \cdots n_{m}=\square}} \frac{\prod_{p \mid n_{1} m}\left(1+p^{-1}\right)^{-1}}{n_{1}^{s_{1}} \cdots n_{m}^{s_{m}}}
$$

for the cases $v=2,3$.

The completion of the proof of Proposition 3.3 now immediately follows from equations (3.9), (3.19) and (3.20) after separating the cases when the product $n_{1} \cdots n_{m}$ is even or odd.

PROPOSITION 3.5. Let $\Re_{\left(s_{i}\right)}$ be sufficiently large for $i=1,2, \ldots, m$. Then

$$
\operatorname{Res}_{w=1}\left[\zeta(2 w) \cdot Z^{+}\left(s_{1}, \ldots, s_{m}, w\right)\right]=\frac{1}{2} R\left(s_{1}, \ldots, s_{m}\right) \cdot \prod_{i=1}^{m} \zeta\left(2 s_{i}\right) \prod_{1 \leqslant i<j \leqslant m} \zeta\left(s_{i}+s_{j}\right),
$$

where $R\left(s_{1}, \ldots, s_{m}\right)$ can be holomorphically continued to the region $\Re\left(s_{i}\right)>\frac{1}{2}-\epsilon$ for some fixed $\epsilon>0$. Further, $R\left(\frac{1}{2}, \ldots, \frac{1}{2}\right)=a_{m}$, where $a_{m}$ is the constant given in (3.3).

Proof. If $f(n)$ is a multiplicative function for which the sum $\sum_{n=1}^{\infty} f(n)$ converges absolutely, then we have the Euler product identity

$$
\sum_{n=1}^{\infty} f(n)=\prod_{p}\left(1+f(p)+f\left(p^{2}\right)+f\left(p^{3}\right)+\cdots\right)
$$

It now follows from Proposition 3.3 and (3.20) that

$$
\operatorname{Res}_{w=1}\left[\zeta(2 w) Z^{+}\left(s_{1}, \ldots, s_{m}, w\right)\right]=\frac{1}{2} \prod_{p}\left[1+\left(1+\frac{1}{p}\right)^{-1} \sum_{\substack{\mu=1 \\ e^{2}}}^{\infty} \sum_{\substack{e_{1}+\cdots+e_{m}=2 \mu \\ e_{i} \geqslant 0,(i=1, \ldots, m)}} p^{-\left(e_{1} s_{1}+\cdots+e_{m} s_{m}\right)}\right],
$$

where the product converges for $\Re_{\left(s_{i}\right)}>\frac{1}{2}$, (for $\left.i=1,2, \ldots, m\right)$. On the other hand, the function $R\left(s_{1}, \ldots, s_{m}\right)$ defined by 


$$
\prod_{p}\left[1+\left(1+\frac{1}{p}\right)^{-1} \sum_{\substack{\mu=1 \\ e_{i} \geqslant 0,(i=1, \ldots, m)}}^{\infty} \sum_{\substack{e_{1}+\ldots+e_{m}=2 \mu \\ e_{i} \geqslant 0}} p^{-\left(e_{1} s_{1}+\cdots+e_{m} s_{m}\right)}\right] \prod_{i=1}^{m} \zeta\left(2 s_{i}\right)^{-1} \prod_{1 \leqslant i<j \leqslant m} \zeta\left(s_{i}+s_{j}\right)^{-1}
$$

is holomorphic for $\Re\left(s_{i}\right)>\frac{1}{2}-\epsilon,(i=1,2, \ldots, m)$ for some fixed small $\epsilon>0$. This establishes the first part of Proposition 3.5.

Now, the number of terms in the inner sum

$$
\sum_{\substack{e_{1}+\cdots+e_{m}=2 \mu \\ e_{i} \geqslant 0,(i=1, \ldots, m)}} p^{-\left(e_{1} s_{1}+\cdots+e_{m} s_{m}\right)}
$$

of formula (3.21) is precisely

$$
d_{m}\left(p^{2 \mu}\right)=\frac{(m+2 \mu-1) !}{(m-1) ! \cdot(2 \mu) !} .
$$

If we specialize to $s_{1}=\cdots=s_{m}=s$, we get

$$
\begin{aligned}
& \prod_{p}\left[1+\left(1+\frac{1}{p}\right)^{-1} \sum_{\mu=1}^{\infty} \sum_{\substack{e_{1}+\ldots+e_{m}=2 \mu \\
e_{i} \geqslant 0,(i=1, \ldots, m)}} p^{-\left(e_{1}+\ldots .+e_{m}\right)^{s}}\right] \\
& =\prod_{p}\left[1+\left(1+\frac{1}{p}\right)^{-1} \sum_{\mu=1}^{\infty} d_{m}\left(p^{2 \mu}\right) p^{-2 \mu s}\right] .
\end{aligned}
$$

It follows from (3.21) that for $\Re(s) \geqslant \frac{1}{2}$,

$$
R\left(s_{1}, \ldots, s\right)=\prod_{p}\left[1+\left(1+\frac{1}{p}\right)^{-1} \sum_{\mu=1}^{\infty} d_{m}\left(p^{2 \mu}\right) p^{-2 \mu s}\right] \cdot \zeta(2 s)^{-M}
$$

and

$$
R\left(\frac{1}{2}, \ldots, \frac{1}{2}\right)=\prod_{p}\left[\left(1-\frac{1}{p}\right)^{M}\left(1+\left(1+\frac{1}{p}\right)^{-1} \sum_{\mu=1}^{\infty} d_{m}\left(p^{2 \mu}\right) p^{-\mu}\right)\right] .
$$

If we apply the binomial formula to $\left(1-p^{-\frac{1}{2}}\right)^{-m}+\left(1+p^{-\frac{1}{2}}\right)^{-m}$ in the definition of $a_{m}$ given in (3.3) we obtain $R\left(\frac{1}{2}, \ldots, \frac{1}{2}\right)=a_{m}$. This completes the proof of Proposition 3.5. 


\subsection{A FUNDAMENTAL CONJECTURE FOR THE FAMILY OF QUADRATIC DIRICHLET $L$-FUNCTIONS}

In view of the invariance of $\widehat{Z^{ \pm}}$under the group $G_{m}$, it follows (as in Section 2.3) from Proposition 3.5 that the polar divisors of $Z^{ \pm}$must contain the $2^{m}$ hyperplanes

$$
\epsilon_{1} s_{1}+\cdots+\epsilon_{m} s_{m}+w-\frac{\epsilon_{1}+\cdots+\epsilon_{m}+2}{2}=0,
$$

where each $\epsilon_{i}=0$ or 1 for $i=1, \ldots, m$. All the hyperplanes (3.22) pass through the point $\left(\frac{1}{2}, \ldots, \frac{1}{2}, 1\right)$. We propose the following conjecture.

CONJECTURE 3.6. The functions $\widehat{Z^{ \pm}}$have meromorphic continuation to a tube domain in $\mathbb{C}^{m+1}$ which contains the point $\left(\frac{1}{2}, \ldots, \frac{1}{2}, 1\right)$, and both these functions have the same polar divisor. The part of the polar divisor passing through $\left(\frac{1}{2}, \ldots, \frac{1}{2}, 1\right)$ consists of all the hyperplanes (3.22). Moreover, the functions $Z^{ \pm}\left(\frac{1}{2}, \ldots, \frac{1}{2}, w\right)$ are holomorphic for $\Re(w)>1$.

THEOREM 3.7 For $m$ even, Conjecture 3.6 implies the Keating-Snaith-ConreyFarmer Conjecture 3.1.

Proof. We need to again apply Stark's version of the Wiener-Ikehara Tauberian theorem as quoted in the proof of Theorem 2.8. Here we take $S(x)=\sum_{|d| \leqslant x}$ $L\left(1 / 2, \chi_{d}\right)^{m}$. Writing $S(x)$ as a Riemann-Stieltjes integral, it follows by integration by parts, that

$$
\int_{1}^{\infty} S(t) \cdot t^{-w} \frac{\mathrm{d} t}{t}=\frac{1}{w} \sum_{d} \frac{L\left(\frac{1}{2}, \chi_{d}\right)^{m}}{|d|^{w}} .
$$

Since we have assumed $m$ to be even, it follows from (3.8), (3.9) that $Z^{ \pm}\left(\frac{1}{2}, \ldots, \frac{1}{2}, w\right)$ is a Dirichlet series satisfying the conditions of the Tauberian theorem. To prove Conjecture 3.1, it is enough to show that

$$
\lim _{w \rightarrow 1}(w-1)^{M+1} Z^{ \pm}\left(\frac{1}{2}, \ldots, \frac{1}{2}, w\right)=\frac{3}{\pi^{2}} g_{m} a_{m} M !,
$$

where

$$
M=\frac{m(m+1)}{2}, \quad g_{m}=\prod_{\ell=1}^{m} \frac{\ell !}{(2 \ell) !},
$$

and $a_{m}$ is the constant given in (3.3).

Let $T\left(s_{1}, \ldots, s_{m}, w\right)$ denote the function defined by

$$
\frac{1}{2(w-1)} R\left(s_{1}, \ldots, s_{m}\right) \prod_{i=1}^{m} \pi^{-\frac{s_{i}+a}{2}} \Gamma\left(\frac{s_{i}+a}{2}\right) \zeta\left(2 s_{i}\right) \prod_{1 \leqslant i<j \leqslant m} \zeta\left(s_{i}+s_{j}\right),
$$

where $a=0,1$ is determined by $(-1)^{a}= \pm 1$. Then Conjecture 3.6 implies that

$$
\zeta(2 w) \widehat{Z^{ \pm}}\left(s_{1}, \ldots, s_{m}, w\right)-\sum_{\alpha \in G_{m}} T\left(\alpha\left(s_{1}, \ldots, s_{m}, w\right)\right)
$$


is holomorphic around $\left(\frac{1}{2}, \ldots, \frac{1}{2}, 1\right)$. The Proof of Theorem 3.7 is an immediate consequence of the following Proposition.

PROPOSITION 3.8. For $m=1,2,3, \ldots$, let $G_{m}$ denote the direct product of $m$ groups of order 2 generated by the involutions (3.7). Let

$$
U\left(s_{1}, \ldots, s_{m}, w\right)=\frac{1}{w-1} R\left(s_{1}, \ldots, s_{m}\right) \prod_{i=1}^{m} \zeta\left(2 s_{i}\right) \prod_{1 \leqslant i<j \leqslant m} \zeta\left(s_{i}+s_{j}\right) .
$$

Then we have

$$
\lim _{w \rightarrow 1} \lim _{\left(s_{1}, \ldots, s_{m}\right) \rightarrow\left(\frac{1}{2}, \ldots, \frac{1}{2}\right)}\left[(w-1)^{M+1} \sum_{\alpha \in G_{m}} U\left(\alpha\left(s_{1}, \ldots, s_{m}, w\right)\right)\right]=\frac{6}{\pi^{2}} a_{m} g_{m} M !,
$$

where

$$
M=\frac{m(m+1)}{2}, \quad g_{m}=\prod_{\ell=1}^{m} \frac{\ell !}{(2 \ell) !},
$$

and $a_{m}$ is the constant given in (3.3).

Proof. We start by taking the Taylor expansion of

$$
U\left(s_{1}, \ldots, s_{m}, w\right)=\frac{a_{m}}{w-1} \cdot \frac{f^{*}\left(s_{1}, \ldots, s_{m}\right)}{\prod_{i=1}^{m}\left(2 s_{i}-1\right) \prod_{1 \leqslant i<j \leqslant m}\left(s_{i}+s_{j}-1\right)}
$$

around $\left(s_{1}, \ldots, s_{m}\right)=\left(\frac{1}{2}, \ldots, \frac{1}{2}\right)$. Here

$$
f^{*}\left(s_{1}, \ldots, s_{m}\right)=1+\sum_{\substack{\ell_{1}=0 \\ \ell_{1}+\cdots+\ell_{m} \geqslant 1}}^{\infty} \ldots \sum_{\substack{\ell_{m}=0 \\ \infty}}^{\infty} \kappa_{m}\left(\ell_{1}, \ldots, \ell_{m}\right)\left(s_{1}-\frac{1}{2}\right)^{\ell_{1}} \cdots\left(s_{m}-\frac{1}{2}\right)^{\ell_{m}},
$$

(with $\kappa_{m}\left(\ell_{1}, \ldots, \ell_{m}\right) \in \mathbb{C}$ ), will be a holomorphic function which is symmetric function with respect to the variables $s_{1}, \ldots, s_{m}$.

Now, make the change of variables $s_{i}=\frac{1}{2}+\epsilon_{i}$ for $i=1,2, \ldots, m$, and $w=v+1$. The involutions (3.7) are transformed to

$$
\left(\epsilon_{1}, \ldots, \epsilon_{i}, \ldots, \epsilon_{m}, v\right) \stackrel{\alpha_{i}}{\longrightarrow}\left(\epsilon_{1}, \ldots,-\epsilon_{i}, \ldots, \epsilon_{m}, v+\epsilon_{i}\right), \quad(i=1,2, \ldots, m) .
$$

Henceforth, we denote by $G_{m}$ the group generated by the above involutions.

Then by (3.25), it is enough to prove that

$$
\lim _{v \rightarrow 0} \lim _{\left(\epsilon_{1}, \ldots, \epsilon_{m}\right) \rightarrow(0, \ldots, 0)}\left[v^{M+1} \sum_{\alpha \in G_{m}} H_{f}\left(\alpha\left(\epsilon_{1}, \ldots, \epsilon_{m}, v\right)\right)\right]=2^{m} g_{m} M !
$$

where

$$
H_{f}\left(\epsilon_{1}, \ldots, \epsilon_{m}, v\right)=\frac{1}{v} \cdot \frac{f\left(\epsilon_{1}, \ldots, \epsilon_{m}\right)}{\prod_{i=1}^{m} \epsilon_{i} \prod_{1 \leqslant i<j \leqslant m}\left(\epsilon_{1}+\epsilon_{j}\right)},
$$


and $f$ (which is simply related to $f^{*}$ ) is a certain holomorphic symmetric function with respect to the variables $\epsilon_{1}, \ldots, \epsilon_{m}$. It satisfies $f(0, \ldots, 0)=1$.

The proof of Proposition 3.8 is an immediate consequence of the following two lemmas.

LEMMA 3.9. The limit (3.26) exists.

Proof. Let $f=\sum_{k \geqslant 0} f_{k}$, where $f_{k}$ (for $k=0,1,2, \ldots$ ) is a symmetric and homogeneous polynomial of degree $k$. Here $f_{0}=1$. It follows that $H_{f}=\sum_{k \geqslant 0} H_{f_{k}}$. Since the action of the group $G_{m}$ commutes with permutations of the variables $\epsilon_{1}, \ldots, \epsilon_{m}$, it easily follows that

$$
\sum_{\alpha \in G_{m}} H_{f_{k}}\left(\alpha\left(\epsilon_{1}, \ldots, \epsilon_{m}, v\right)\right)
$$

is also a symmetric function with respect to $\epsilon_{1}, \ldots, \epsilon_{m}$.

Define

$$
N_{f_{k}}\left(\epsilon_{1}, \ldots, \epsilon_{m}, v\right)=\left(\prod_{i=1}^{m} \epsilon_{i} \prod_{i \leqslant i<j \leqslant m}\left(\epsilon_{i}^{2}=\epsilon_{j}^{2}\right)\right) \sum_{\alpha \in G_{m}} H_{f_{k}}\left(\alpha\left(\epsilon_{1}, \ldots, \epsilon_{m}, v\right)\right) .
$$

Then $N_{f_{k}}$ is a symmetric function in the variables $\epsilon_{1}, \ldots, \epsilon_{m}$, and it is a rational function

$$
N_{f_{k}}=\frac{N_{f_{k}}^{*}}{D_{f_{k}}^{*}}
$$

with denominator of the form

$$
D_{f_{k}}^{*}\left(\epsilon_{1}, \ldots, \epsilon_{m}, v\right)=\prod_{\delta_{1}=0}^{1} \cdots \prod_{\delta_{m}=0}^{1}\left(v+\delta_{1} \epsilon_{1}+\cdots+\delta_{m} \epsilon_{m}\right) .
$$

It follows that

$$
N_{f_{k}}\left(\ldots, \epsilon_{i}, \ldots, \epsilon_{j}, \ldots, v\right)=-N_{f_{k}}\left(\ldots, \epsilon_{j}, \ldots, \epsilon_{i}, \ldots, v\right),
$$

which implies that

$$
N_{f_{k}}\left(\ldots, \epsilon_{i}, \ldots, \epsilon_{j}, \ldots, v\right)=0 .
$$

This gives

$$
\left(\epsilon_{j}-\epsilon_{j}\right) \mid N_{f_{k}}^{*}\left(\epsilon_{1}, \ldots, \epsilon_{m}, v\right) .
$$

Furthermore, since $\sum_{\alpha \in G_{m}} H_{f_{k}}\left(\alpha\left(\epsilon_{1}, \ldots, \epsilon_{m}, v\right)\right)$ is invariant under the group $G_{m}$, it follows that

$$
N_{f_{k}}\left(\ldots, s_{i}, \ldots, v\right)=-N_{f_{k}}\left(\ldots,-s_{i}, \ldots, v+s_{i}\right)
$$

which implies that $N_{f_{k}}(\ldots, 0, \ldots, v)=0$. Consequently,

$$
s_{i} \mid N_{f_{k}}^{*}\left(\epsilon_{1}, \ldots, \epsilon_{m}, v\right) \text {. }
$$


Also, in the same manner, (3.29) and (3.31) imply that

$$
\left.\left(s_{i}+s_{j}\right) \mid N_{f_{k}}^{*}\left(\epsilon_{1}, \ldots, \epsilon_{m}, v\right)\right) .
$$

Finally, it follows from (3.27), (3.28), (3.30), (3.32), and (3.33) that for $\Re(v)>0$, the limit

$$
\lim _{\left(\epsilon_{1}, \ldots, \epsilon_{m}\right) \rightarrow(0, \ldots, 0)} \sum_{\alpha \in G_{m}} H_{f_{k}}\left(\alpha\left(\epsilon_{1}, \ldots, \epsilon_{m}, v\right)\right)
$$

exists. It further follows that if we set $\epsilon_{i}=i \cdot \epsilon$ (for $i=1,2 \ldots, m$ ) then for $K=0,1,2,3, \ldots$,

$$
\sum_{\alpha \in G_{m}} H_{f_{k}}\left(\alpha\left(\epsilon_{1}, \ldots, \epsilon_{m}, v\right)\right)=\frac{P_{k}(\epsilon, v)}{\prod_{\ell=0}^{M}(v+\ell \epsilon)},
$$

where $P_{k}(\epsilon, v)$ is a homogeneous polynomial of degree $k$ in the two variables $\epsilon, v$.

Consequently

$$
\lim _{v \rightarrow 0} \lim _{\epsilon \rightarrow 0} v^{M+1} \sum_{\alpha \in G_{m}} H_{f_{k}}\left(\alpha\left(\epsilon_{1}, \ldots, \epsilon_{m}, v\right)\right)=0
$$

if $k>0$, and the limit exists if $k=0$. This completes the proof of Lemma 3.9.

LEMMA 3.10. Let

$$
H\left(\epsilon_{1}, \ldots, \epsilon_{m}, v\right)=\frac{1}{v} \cdot \frac{1}{\prod_{i=1}^{m} \epsilon_{i} \prod_{1 \leqslant i<j \leqslant m}\left(\epsilon_{i}+\epsilon_{j}\right)} .
$$

Then

$$
\lim _{v \rightarrow 0} \lim _{\left(\epsilon_{1}, \ldots, \epsilon_{m}\right) \rightarrow(0, \ldots, 0)} \sum_{\alpha \in G_{m}} H\left(\alpha\left(\epsilon_{1}, \ldots, \epsilon_{m}, v\right)\right)=2^{m} g_{m} M ! .
$$

Proof. We know from Lemma 3.9 that the above limit exists, so we can compute the limit by setting $\epsilon_{j}=j \epsilon$ (for $j=1,2, \ldots, m$ ) and letting $\epsilon \rightarrow 0$. It follows from (3.34) that

$$
\sum_{\alpha \in G_{m}} H(\alpha(\epsilon, 2 \epsilon, \ldots, m \epsilon, v))=\frac{\kappa_{m}}{\prod_{\ell=0}^{M}(v+\ell \epsilon)}
$$

for some constant $\kappa_{m}$. By taking the reside at $w=0$ on both sides, we have

$$
\frac{1}{m !} \prod_{1 \leqslant i<j \leqslant m} \frac{1}{(i+j)}=\frac{\kappa_{m}}{M !} .
$$

By induction over $m$, one can show that $\kappa_{m}=2^{m} g_{m} M$ !, and the lemma follows. 


\section{Cubic Moments of Quadratic $L$-series}

As mentioned in the introduction, in the particular cases when $m \leqslant 3$ it is possible to define an analog of the multiple Dirichlet series given in (3.6). In this analog the sum is not restricted to fundamental discriminants, but ranges over all integers $d$. When an appropriate definition is given for $\prod_{i=1}^{m} L\left(s_{i}, \chi_{d}\right)$ for general $d$ one can extend the multiple Dirichlet series to a meromorphic function of $s_{1}, s_{2}, \ldots, s_{m}, w$ in $\mathrm{C}^{m+1}$. In this section we will explicitly provide this continuation in the case $m=3$ and $s_{1}=s_{2}=s_{3}=s$. This work relies heavily on the results of [B-F-H-1]. We will then develop a sieving method analogous to that used in [G-H] to isolate fundamental discriminants and will prove as a consequence Theorem 1.1.

\subsection{SOME FOUNDATIONS}

The $L$ series $\zeta(s)^{3}$ can actually be associated to a certain Eisenstein series $F$ on GL(3), and $L(s, F)=\zeta(s)^{3}$.

For future convenience, we will write

$$
L(s, F)=\sum_{1}^{\infty} \frac{c(n)}{n^{s}},
$$

where $c(n)=\sum_{d_{1} d_{2} d_{3}=n} 1$, and we have the Euler product decomposition

$$
L(s, F)=\prod_{p}\left(1-p^{-s}\right)^{-3},
$$

the product being over all primes $p$ of $Q$.

As in the previous sections, let $\chi_{d}$ denote the primitive quadratic character associated to the quadratic field $Q(\sqrt{d})$. If $F$ is twisted by $\chi_{d}$, then the associated $L$-series becomes

$$
L\left(s, F, \chi_{d}\right)=L\left(s, \chi_{d}\right)^{3}=\prod_{p}\left(1-\chi_{d}(p) p^{-s}\right)^{-3},
$$

and by (3.12) the functional equation is given by

$$
\left(|D|^{3}\right)^{s / 2} G_{d}(s) L\left(s, F, \chi_{d}\right)=\left(|D|^{3}\right)^{(1-s) / 2} G_{d}(1-s) L\left(1-s, F, \chi_{d}\right) .
$$

Here $D=4 d$ or $D=d$ is the conductor of $\chi_{d}$ and $G_{d}(s)$ denotes the product of gamma factors.

The gamma factors (4.4), described in (3.12), depend only on the sign of $d$. Although we will not require many explicit properties of the gamma factors, the following upper bound will be convenient. For $\sigma_{1}>\sigma_{2}$ and $t$ real, it follows from Stirling's formula that for large $|t|$, independent of $d$,

$$
\frac{\left|G_{d}\left(\sigma_{1}+i t\right)\right|}{\left|G_{d}\left(\sigma_{2}-i t\right)\right|} \ll(|t|+1)^{3\left(\sigma_{1}-\sigma_{2}\right) / 2}
$$

When all primes are included in the product (4.3) the functional equation (4.4) has its optimal form. However, it is often convenient to omit factors corresponding to 
'bad' primes, for example those contained in $S$, a finite set of primes including 2 . Let $M=\prod_{p \in S} p$. For such $M, S$, we denote the $L$-series with Euler factors corresponding to primes dividing $M$ removed as follows:

$$
L_{M}(s, F)=\prod_{p \notin S}\left(1-p^{-s}\right)^{-3}=L(s, F) \prod_{p \in S}\left(1-p^{-s}\right)^{3} .
$$

When twisted by $\chi_{d}$, the $L$-series $L\left(s, F, \chi_{d}\right)$ will have a perfect functional equation of the form (4.4) when $\chi_{d}$ is a primitive character. This corresponds to the case where $d$ is square free. It is very interesting to note that often, when $d$ is not square free, it is possible to complete $L\left(s, F, \chi_{d}\right)$ by multiplying by a certain Dirichlet polynomial in such a way that the resulting product has a functional equation of precisely the same form (4.4), with $D$ replaced by $|d|$ or $|4 d|$. For the simplest example, with $m=1$, see [G-H]. What is more remarkable is the fact that some very stringent additional conditions can be imposed on the Dirichlet polynomial.

To be more precise, let $l_{1}, l_{2}>0, l_{1}, l_{2} \mid M$, and $a_{1}, a_{2} \in\{1,-1\}$ and let $\chi_{a_{1} l_{1}}, \chi_{a_{2} l_{2}}$ be the quadratic characters corresponding to $a_{1} l_{1}, a_{2} l_{2}$ as defined above. We then formulate the following collection of properties for two classes of Dirichlet polynomials associated to $F$.

PROPERTY 4.1. For $n, d$ positive integers, $(n d, M)=1$, we write $d=d_{0} d_{1}^{2}, n=n_{0} n_{1}^{2}$, with $d_{0}, n$ square free and $d_{1}, n_{1}$ positive. Let $c(n)$ denote the coefficients of $L(s, F)$ as defined earlier.

For complex numbers $A_{d, p^{e}}^{(\alpha)}, B_{d, p^{e}}^{(\alpha)}$ (depending on $d, \alpha \in Z, 1 \leqslant e \leqslant \alpha$ ), let $P_{d_{0}, d_{1}}^{\left(a_{1} l_{1}\right)}(s)$, $Q_{n_{0}, n_{1}}^{\left(a_{2} l_{2}\right)}(w)$ be Dirichlet polynomials defined by

$$
P_{d_{0}, d_{1}}^{\left(a_{1} l_{1}\right)}(s)=\prod_{p^{\alpha} \| d_{1}}\left(1+A_{d_{0} \cdot a_{1} l_{1}, p}^{(\alpha)} p^{-s}+\cdots+A_{d_{0} \cdot a_{1} l_{1}, p^{6 \alpha}}^{(\alpha)} p^{-6 \alpha s}\right)
$$

and

$$
c\left(n_{0} n_{1}^{2}\right) Q_{n_{0}, n_{1}}^{\left(a_{2} l_{2}\right)}(w)=c\left(n_{0} n_{1}^{2}\right) \prod_{p^{\beta} \| n_{1}}\left(1+B_{n_{0} \cdot a_{2} l_{2}, p}^{(\beta)} p^{-w}+\cdots+B_{n_{0} \cdot a_{2} l_{2}, p^{2 \beta}}^{(\beta)} p^{-2 \beta w}\right) .
$$

We say that $P, Q$ satisfy the conditions of Property 4.1 if the following identities hold:

$$
\begin{aligned}
& d_{1}^{3 s} P_{d_{0}, d_{1}}^{\left(a_{1} l_{1}\right)}(s)=d_{1}^{3(1-s)} P_{d_{0}, d_{1}}^{\left(a_{1} l_{1}\right)}(1-s), \\
& n_{1}^{w} c\left(n_{0} n_{1}^{2}\right) Q_{n_{0}, n_{1}}^{\left(a_{2} l_{2}\right)}(w)=n_{1}^{1-w} c\left(n_{0} n_{1}^{2}\right) Q_{n_{0}, n_{1}}^{\left(a_{2} l_{2}\right)}(1-w) \\
& P_{d_{0} l_{3}, d_{1}}^{\left(a_{1} l_{1}\right)}(s)=P_{d_{0}, d_{1}}^{\left(a_{1} l_{1} l_{3}\right)}(s), \quad Q_{n_{0} l_{3}, n_{1}}^{\left(a_{2} l_{1}\right)}(w)=Q_{n_{0}, n_{1}}^{\left(a_{2} l_{2} l_{3}\right)}(w),
\end{aligned}
$$


(where $d_{0} l_{3}, n_{0} l_{3}$ are positive square free numbers), and if in addition, the following interchange of summation is valid for $s$ and w having sufficiently large real parts:

$$
\begin{aligned}
\sum_{(d, M)=1} \frac{L_{M}\left(s, F, \chi_{d_{0}} \chi_{a_{1} l_{1}}\right) \chi_{a_{2} l_{2}}\left(d_{0}\right) P_{d_{0}, d_{1}}^{\left(a_{1} l_{1}\right)}(s)}{d^{w}} & \\
& =\sum_{(n, M)=1} \frac{L_{M}\left(w, \tilde{\chi}_{n_{0}} \chi_{a_{2} l_{2}}\right) \chi_{a_{1} l_{1}}\left(n_{0}\right) c\left(n_{0} n_{1}^{2}\right) Q_{n_{0}, n_{1}}^{\left(a_{2} l_{2}\right)}(w)}{n^{s}} .
\end{aligned}
$$

Here $\widetilde{\chi}_{n_{0}}$ denotes the quadratic character with conductor $n_{0}$ defined by $\widetilde{\chi}_{n_{0}}(*)=\left(\frac{*}{n_{0}}\right)$. Recall $2 \mid M$, so $\left(2, n_{0}\right)=1$.

It was observed in [B-F-H-1] that the three properties (4.7), (4.8) and (4.10) were sufficient to determine the polynomials $P$ and $Q$, precisely, in the cases of GL(1), GL(2), GL(3). This unique determination of $P$ and $Q$ corresponded to a finite group of functional equations of the double Dirichlet series given in (4.10) and this in turn made it possible to obtain an analytic continuation of the double Dirichlet series in these three cases. It was also noted that for $m \geqslant 4$ the corresponding group of functional equations becomes infinite and that simultaneously the polynomials $P, Q$ are no longer uniquely determined by the properties (4.7), (4.8), and (4.10). The space of local solutions becomes one-dimensional in the case $m=4$, and higher for $m>4$.

In [B-F-H-1] a complete description of certain factors of the polynomials $P, Q$ was obtained for the case of $m=3$ and an arbitrary automorphic form $f$ on GL(3). These were the factors corresponding to the 'good' primes, i.e., primes not dividing 2 or the level of $f$. It was also verified that for sums over positive integers $n, d$ relatively prime to the 'bad' primes, the relations (4.7), (4.8), (4.9), and (4.10) hold. In addition, it was verified that for fixed $d=d_{0} d_{1}^{2}$ and $\epsilon>0, \Re(s) \geqslant \frac{1}{2}$.

$$
P_{d_{0}, d_{1}}^{\left(a_{1} l_{1}\right)}(s) \ll|d|^{\epsilon} .
$$

The implied constant depends only on $\epsilon$. This bound was then used to obtain the analytic continuation of the double Dirichlet series on the left hand side of (4.10). As a consequence, non vanishing results for quadratic twists of $L\left(\frac{1}{2}, f, \chi_{d}\right)$ were obtained and also, after taking a residue at $w=1$, a new proof was obtained for the analytic continuation of the symmetric square of $f$.

As the technique is new, there may be some advantage to presenting the details of the analytic continuation argument specialized to the very concrete case where $L\left(s, f, \chi_{d}\right)=L\left(s, F, \chi_{d}\right)=L\left(s, \chi_{d}\right)^{3}$, and we will do so below.

\subsection{THE CUBIC MOMENT, CONTINUED}

Our object will be to obtain the analytic continuation in $(s, w)$, with $\mathfrak{R}(s) \geqslant \frac{1}{2}, \mathfrak{R}(w)>\frac{4}{5}$, and an estimate for the growth in vertical strips $w=v+i$ (for fixed $v$ and $s$ ) of the double Dirichlet series

$$
Z(s, w)=\sum_{D=\text { fund. disc }} \frac{L\left(s, \chi_{D}\right)^{3}}{|D|^{w}} .
$$


To accomplish this, we will obtain the analytic properties of a building block: For $l_{1}, l_{2}>0, l_{1}, l_{2} \mid M$ and $a_{1}, a_{2} \in\{1,-1\}$, we define

$$
Z_{M}\left(s, w ; \chi_{a_{2} l_{2}}, \chi_{a_{1} l_{1}}\right)=\sum_{(d, M)=1} \frac{L_{M}\left(s, F, \chi_{d_{0}} \chi_{a_{1} l_{1}}\right) \chi_{a_{2} l_{2}}\left(d_{0}\right) P_{d_{0}, d_{1}}^{\left(a l_{1} l_{1}\right)}(s)}{d^{w}},
$$

where we recall that we sum over $d \geqslant 1$ and use the decomposition $d=d_{0} d_{1}^{2}$, with $d_{0}$ square free and $d_{1}$ positive.

The following proposition will provide a useful way of collecting the properties of the multiple Dirichlet series $Z_{M}\left(s, w ; \chi_{a_{2} l_{2},} \chi_{a_{1} l_{1}}\right)$. For a positive integer $M$, define

$$
\operatorname{Div}(M)=\{a \cdot l|a= \pm 1,1 \leqslant l, l| M\},
$$

which has cardinality $2 d(M)=2 \sum_{d \mid M} 1$. Let $\vec{Z}_{M}\left(s, w ; \chi_{a_{2} l_{2},}, \chi_{\operatorname{Div}(M)}\right)$ denote the $2 d(M)$ by 1 column vector whose $j$ th entry is $Z_{M}\left(s, w ; \chi_{a_{2} l_{2}}, \chi^{(j)}\right)$, where $\chi^{(j)}$ $(j=1,2, \ldots, 2 d(M))$ ranges over the characters $\chi_{a_{1} l_{1}}$ with $a_{1}= \pm 1,1 \leqslant l_{1}, l_{1} \mid M$. Then, we will prove

PROPOSITION 4.2. There exists a $2 d(M)$ by $2 d(M)$ matrix $\Phi^{\left(a_{2} l_{2}\right)}(w)$ such that for any fixed $w, w \neq 1$, and for any $s$ with sufficiently large real part (depending on $w$ )

$$
\begin{aligned}
& \prod_{p \mid\left(M / l_{2}\right)}\left(1-p^{-2+2 w}\right) \cdot \vec{Z}_{M}\left(s, w ; \chi_{a_{2} l_{2}}, \chi_{\operatorname{Div}(M)}\right) \\
& \quad=\Phi^{\left(a_{2} l_{2}\right)}(w) \vec{Z}_{M}\left(s+w-1 / 2,1-w ; \chi_{a_{2} l_{2}}, \chi_{\operatorname{Div}(M)}\right) .
\end{aligned}
$$

The entries of $\Phi^{\left(a_{2} l_{2}\right)}(w)$, denoted by $\Phi_{i, j}^{\left(a_{2} l_{2}\right)}(w)$, are meromorphic functions in $\mathrm{C}$.

Proof. By Property 4.1,

$$
Z_{M}\left(s, w ; \chi_{a_{2} l_{2}}, \chi_{a_{1} l_{1}}\right)=\sum_{(n, M)=1} \frac{L_{M}\left(w, \tilde{\chi}_{n_{0}} \chi_{a_{2} l_{2}}\right) \chi_{a_{1} l_{1}}\left(n_{0}\right) c\left(n_{0} n_{1}^{2}\right) Q_{n_{0}, n_{1}}^{\left(a_{2} l_{2}\right)}(w)}{n^{s}} .
$$

Now

$$
L_{M}\left(w, \tilde{\chi}_{n_{0}} \chi_{a_{2} l_{2}}\right)=L_{M}\left(w, \tilde{\chi}_{n_{0}} \chi_{a_{2} l_{2}}\right) \cdot \prod_{p \mid M}\left(1-\tilde{\chi}_{n_{0}} \chi_{a_{2} l_{2}}(p) p^{-w}\right),
$$

where $L_{M}\left(w, \widetilde{\chi}_{n_{0}} \chi_{a_{2} l_{2}}\right)$ satisfies the functional equation

$$
G_{\epsilon}(w)\left(n_{0} l_{2} D_{a_{2} l_{2}}\right)^{w / 2} L\left(w, \tilde{\chi}_{n_{0}} \chi_{a_{2} l_{2}}\right)=G_{\epsilon}(1-w)\left(n_{0} l_{2} D_{a_{2} l_{2}}\right)^{(1-w) / 2} L\left(1-w, \tilde{\chi}_{n_{0}} \chi_{a_{2} l_{2}}\right) .
$$

Here $\epsilon=\widetilde{\chi}_{n_{0}} \chi_{a_{2} l_{2}}(-1)$,

$$
G_{\epsilon(w)}= \begin{cases}\pi^{-w / 2} \Gamma(w / 2) & \text { if } \quad \epsilon=1, \\ \pi^{(w+1) / 2} \Gamma((w+1) / 2) & \text { if } \quad \epsilon=-1,\end{cases}
$$

and

$$
D_{a_{2} l_{2}}= \begin{cases}1 & \text { if } a_{2} l_{2} \equiv 1(\bmod 4) \\ 4 & \text { otherwise }\end{cases}
$$


Combining this with the functional equation for $Q$ given in (4.8), we obtain

$$
\begin{aligned}
Z_{M}\left(s, w ; \chi_{a_{2} l_{2}}, \chi_{a_{1} l_{1}}\right)= & \sum_{a_{3}=1,-1} \sum_{(n, M)=1, n \equiv a_{3}(4)} \frac{G_{\epsilon\left(a_{3} a_{2} l_{2}\right)}(1-w)\left(l_{2} D_{a_{e} l_{2}}\right)^{1 / 2-w}}{G_{\epsilon\left(a_{3} a_{2} l_{2}\right)}(w) n^{s+w-1 / 2}} \times \\
& \times \chi_{a_{1} l_{1}}\left(n_{0}\right) L_{M}\left(1-w, \tilde{\chi}_{n_{0}} \chi a_{2} l_{2}\right) c\left(n_{0} n_{1}^{2}\right) Q_{n_{0}, n_{1}}^{\left(a_{2} l_{2}\right)}(1-w) \times \\
& \times \prod_{p \mid\left(M / l_{2}\right)}\left(1-\tilde{\chi}_{n_{0}} \chi_{a_{2} l_{2}}(p) p^{-w}\right) \times \\
& \times \prod_{p \mid\left(M / l_{2}\right)}\left(1-\tilde{\chi}_{n_{0}} \chi_{a_{2} l_{2}}(p) p^{-1+w}\right)^{-1} .
\end{aligned}
$$

Here $\epsilon(a)$ denotes the sign of $a$. Note that we are leaving out terms in the product where $p \mid l_{2}$ as the character vanishes here.

Multiplying by $\prod_{p \mid\left(M / l_{2}\right)}\left(1-p^{-2+2 w}\right)$ and reorganizing, we obtain

$$
\begin{aligned}
& \prod_{p \mid\left(M / l_{2}\right)}\left(1-p^{-2+2 w}\right) \cdot Z_{M}\left(s, w ; \chi_{a_{2} l_{2}}, \chi_{a_{1} l_{1}}\right) \\
& =\sum_{a_{3}=1,-1} \frac{G_{\epsilon\left(a_{3} a_{2} l_{2}\right)}(1-w)}{G_{\epsilon\left(a_{3} a_{2} l_{2}\right)}(w)\left(l_{2} D_{a_{2} l_{2}}\right)^{w-1 / 2}} \sum_{l_{3}, l_{4} \mid\left(M / l_{2}\right),\left(l_{4}, 2\right)=1} \mu\left(l_{3}\right) \chi_{a_{2} l_{2}}\left(l_{3} l_{4}\right) l_{3}^{-w} l_{4}^{-1+w} \times \\
& \quad \times \sum_{\substack{(n, M)=1, n=a_{3}(4)}} \frac{A_{2}\left(1-w, \tilde{\chi}_{n_{0}} \chi_{a_{2} l_{2}}\right) L_{M}\left(1-w, \tilde{\chi}_{n_{0}} \chi_{a_{l_{2}} l_{2}}\right) c\left(n_{0} n_{1}^{2}\right) Q_{n_{0}, n_{1}}^{\left(a_{2} l_{2}\right)}(1-w) \chi_{a_{1} l_{1} l_{3} l_{4}}\left(n_{0}\right)}{n^{s+w-1 / 2}},
\end{aligned}
$$

where

$$
A_{2}\left(w, \tilde{\chi}_{n_{0}} \chi_{a_{2} l_{2}}\right)=\left\{\begin{array}{lll}
1 & \text { if } & 2 \mid l_{2}, \\
1+\tilde{\chi}_{n_{1}} \chi_{a_{2} l_{2}}(2) 2^{-w} & \text { if } & a_{2} l_{2} \equiv 1(\bmod 4), \\
1-2^{-2 w} & \text { if } & a_{2} l_{2} \equiv-1(\bmod 4) .
\end{array}\right.
$$

We have used here the fact that $\widetilde{\chi}_{n_{0}}\left(l_{3}\right) \widetilde{\chi}_{n_{0}}\left(l_{4}\right)=\chi_{l_{3} l_{4}}\left(n_{0}\right)$, and the identity

$$
\left(1-2^{-2+2 w}\right)\left(1-\tilde{\chi}_{n_{0}} \chi_{a_{2} l_{2}}(2) 2^{-1+w}\right)^{-1}=A_{2}\left(1-w, \tilde{\chi}_{n_{0}} \chi_{a_{2} l_{2}}\right),
$$

for $a_{2} l_{2} \equiv-1,1(\bmod 4)$.

Using $\chi_{-1}$ to sieve congruence classes of $n(\bmod 4)$ :

$$
\frac{1}{2}\left(1+a_{3} \chi-1\left(n_{0}\right)\right)=\left\{\begin{array}{lll}
1 & \text { if } & n_{0} \equiv a_{3}(\bmod 4) \\
0 & \text { if } \quad n_{0} \equiv-a_{3}(\bmod 4)
\end{array}\right.
$$

we finally obtain (in the case of $a_{2} l_{2} \equiv 1(\bmod 4)$ )

$$
\begin{aligned}
& \prod_{p \mid\left(M / l_{2}\right)}\left(1-p^{-2+2 w}\right) \cdot Z_{M}\left(s, w ; \chi_{a_{2} l_{2}}, \chi_{a_{1} l_{1}}\right) \\
& =\frac{1}{2} \cdot l_{2}^{1 / 2-w} \cdot \sum_{l_{3}, l_{4} \mid\left(M / l_{2}\right)} \mu\left(l_{3}\right) \chi_{a_{2} l_{2}}\left(l_{3} l_{4}\right) l_{3}^{-w} l_{4}^{-1+w} \sum_{a_{3}=1,-1} \frac{G_{\epsilon\left(a_{3} a_{2} l_{2}\right)}(1-w)}{G_{\epsilon\left(a_{3} a_{2} l_{2}\right)}(w)} \times \\
& \quad \times\left(Z_{M}\left(s+w-1 / 2,1-w ; \chi_{a_{2} l_{2}}, \chi_{a_{1} l_{1} l_{3} l_{4}}\right)+\right. \\
& \left.\quad+a_{3} Z_{M}\left(s+w-1 / 2,1-w ; \chi_{a_{2} l_{2}}, \chi_{-a_{1} l_{1} l_{3} l_{4}}\right)\right) .
\end{aligned}
$$


If $a_{2} l_{2} \equiv-1,2(\bmod 4)$, we have a similar expression. Actually, it can be easily observed that just the behavior at the finite place 2 changes.

This completes the proof of Proposition 4.2.

The function $Z_{M}\left(s, w ; \chi_{a_{2} l_{2}}, \chi_{a_{1} l_{1}}\right)$ defined in (4.13) also possesses a functional equation as $s \rightarrow 1-s$. To describe this, let $d(M)$ be as before, and let $\overrightarrow{\mathbf{Z}}_{M}\left(s, w ; \chi_{\operatorname{Div}(M)}, \chi_{a_{1} l_{1}}\right)$ denote the $2 d(M)$ by 1 column vector whose $j$ th entry is $Z_{M}\left(s, w ; \chi^{(j)}, \chi_{a_{1} l_{1}}\right)$, where $\chi^{(j)}(j=1,2, \ldots, 2 d(M))$ ranges over the characters $\chi_{a_{2} l_{2}}$ with $a_{2}= \pm 1,1 \leqslant l_{2}, l_{2} \mid M$.

Then we have the following.

PROPOSITION 4.3. There exists a $2 d(M)$ by $2 d(M)$ matrix $\Psi^{\left(a_{1} l_{1}\right)}(s)$ such that for any fixed $s, s \neq 1$, and for any $w$ with sufficiently large real part (depending on $s$ )

$$
\begin{aligned}
& \overrightarrow{\mathbf{Z}}_{M}\left(s, w ; \chi_{\operatorname{Div}(M)}, \chi_{a_{1} l_{1}}\right) \cdot \prod_{p \mid\left(M / l_{1}\right)}\left(1-p^{-2+2 s}\right)^{3} \\
& \quad=\Psi^{\left(a_{1} l_{1}\right)}(s) \overrightarrow{\mathbf{Z}}_{M}\left(1-s, w+3 s-3 / 2 ; \chi_{\operatorname{Div}(M)}, \chi_{a_{1} l_{1}}\right) .
\end{aligned}
$$

The entries of $\Psi^{\left(a_{1} l_{1}\right)}(s)$, denoted by $\Psi_{i, j}^{\left(a_{1} l_{1}\right)}(s)$, are meromorphic functions in $\mathrm{C}$.

Proof. First, write

$$
\begin{aligned}
L_{M}\left(s, F, \chi_{d_{0}} \chi_{a_{1} l_{1}}\right) & =L\left(s, F, \chi_{a_{1} d_{0} l_{1}}\right) \cdot \prod_{p \mid\left(M / l_{1}\right)}\left(1-\chi_{a_{1} d_{0} l_{1}}(p) p^{-s}\right)^{3} \\
& =L\left(s, F, \chi_{a_{1} d_{0} l_{1}}\right) \cdot\left(\sum_{l \mid\left(M / l_{1}\right)} \mu(l) \chi_{a_{1} d_{0} l_{1}}(l) l^{-s}\right)^{3} .
\end{aligned}
$$

By (4.4)

$$
L\left(s, F, \chi_{a_{1} d_{0} l_{1}}\right)=\left(d_{0} l_{1} D_{a_{1} d_{0} l_{1}}\right)^{3 / 2-3 s} \frac{G_{\epsilon}(1-s)^{3}}{G_{\epsilon}(s)^{3}} L\left(1-s, F, \chi_{a_{1} d_{0} l_{1}}\right),
$$

where $G_{\epsilon}$ and $D_{a_{1} d_{0} l_{1}}$ is given by (4.17) and $\epsilon$ equals the sign of $a_{1} d_{0} l_{1}$.

On the other side of the functional equation (4.20), we have

$$
L\left(1-s, F, \chi_{a_{1} d_{0} l_{1}}\right)=L_{M}\left(1-s, F, \chi_{a_{1} d_{0} l_{1}}\right) \cdot \prod_{p \mid\left(M / l_{1}\right)}\left(1-\chi_{a_{1} d_{0} l_{1}}(p) p^{-1+s}\right)^{-3} .
$$

In view of the elementary identity

$$
\begin{aligned}
\prod_{p \mid\left(M / l_{1}\right)}\left(1-p^{-2+2 s}\right)= & A_{2}\left(1-s, \chi_{a_{1} d_{0} l_{1}}\right) \prod_{p \mid\left(M / l_{1}\right) p \neq 2}\left(1+\chi_{a_{1} d_{0} l_{1}}(p) p^{-1+s}\right) \times \\
& \times \prod_{p \mid\left(M / l_{1}\right)}\left(1-\chi_{a_{1} d_{l_{1}} l_{1}}(p) p^{-1+s}\right),
\end{aligned}
$$

where

$$
A_{2}\left(s, \chi_{a_{1} d_{0} l_{1}}\right)=\left\{\begin{array}{lll}
1 & \text { if } 2 \mid l_{1}, \\
1+\chi_{a_{1} d_{0} l_{1}}(2) 2^{-s} & \text { if } \quad a_{1} d_{0} l_{1} \equiv 1(\bmod 4), \\
1-2^{-2 s} & \text { if } \quad a_{1} d_{0} l_{1} \equiv-1(\bmod 4),
\end{array}\right.
$$


it immediately follows that

$$
\begin{aligned}
& L\left(1-s, F, \chi_{a_{1} d_{0} l_{1}}\right) \cdot \prod_{p \mid\left(M / l_{1}\right)}\left(1-p^{-2+2 s}\right)^{3} \\
& \quad=L_{M}\left(1-s, F, \chi_{a_{1} d_{0} l_{1}}\right) \cdot A_{2}\left(1-s, F, \chi_{a_{1} d_{0} l_{1}}\right)^{3} \cdot \prod_{\substack{p \mid\left(M / l_{1}\right) \\
p \neq 2}}\left(1+\chi_{a_{1} d_{0} l_{1}}(p) p^{-1+s}\right)^{3} \\
& \quad=L_{M}\left(1-s, F, \chi_{a_{1} d_{0} l_{1}}\right) \cdot A_{2}\left(1-s, \chi_{a_{1} d_{0} l_{1}}\right)^{3} \cdot\left(\sum_{\substack{l\left(M / l_{1}\right) \\
(l, 2)=1}} \chi_{a_{1} d_{0} l_{1}}(l) l^{-1+s}\right)^{3} .
\end{aligned}
$$

Combining the above with (4.7), (4.13), (4.20), we obtain

$$
\begin{aligned}
& Z_{M}\left(s, w ; \chi_{a_{2} l_{2}}, \chi_{a_{1} l_{1}}\right) \cdot \prod_{p \mid\left(M / l_{1}\right)}\left(1-p^{-2+2 s}\right)^{3} \\
& =\sum_{(d, M)=1}\left(l_{1} D_{a_{1} d_{0} l_{1}}\right)^{3 / 2-3 s} \frac{G_{\epsilon\left(a_{1}\right)}(1-s)^{3}}{G_{\epsilon\left(a_{1}\right)}(s)^{3}} \cdot \frac{L_{M}\left(1-s, F, \chi_{a_{1} d_{0} l_{1}}\right)}{d^{w+3 s-3 / 2}} \cdot P_{d_{0}, d_{1}}^{\left(a_{1} l_{1}\right)}(1-s) \chi_{a_{2} l_{2}}\left(d_{0}\right) \times \\
& \quad \times\left(\sum_{l \mid\left(M / l_{1}\right)} \mu(l) \chi_{a_{1} d_{0} l_{1}}(l) l^{-s}\right)^{3} \cdot A_{2}\left(1-s, \chi_{a_{1} d_{0} l_{1}}\right)^{3} \cdot\left(\sum_{\substack{l\left(M / l_{1}\right) \\
(l, 2)=1}} \chi_{a_{1} d_{0} l_{1}}(l) l^{-1+s}\right)^{3} .
\end{aligned}
$$

Write

$$
\begin{aligned}
& \left(\sum_{l \mid\left(M / l_{1}\right)} \mu(l) \chi_{a_{1} d_{0} l_{1}}(l) l^{-s}\right)^{3} \\
& \quad=\sum_{l_{\alpha} \mid\left(M / l_{1}\right)} \mu\left(l_{\alpha}\right) \chi_{a_{1} d_{0} l_{1}}\left(l_{\alpha}\right) l_{\alpha}^{-s} \cdot \sum_{l_{\beta} \mid\left(M / l_{1}\right)} \mu\left(l_{\beta}\right) \chi_{a_{1} d_{0} l_{1}}\left(l_{\beta}\right) l_{\beta}^{-s} \cdot \sum_{l_{\gamma} \mid\left(M / l_{1}\right)} \mu\left(l_{\gamma}\right) \chi_{a_{1} d_{0} l_{1}}\left(l_{\gamma}\right) l_{\gamma}^{-s},
\end{aligned}
$$

and similarly, write

$$
\begin{aligned}
& \left(\sum_{\substack{l\left(M / l_{1}\right) \\
(l, 2)=1}} \chi_{a_{1} d_{0} l_{1}}(l) l^{-1+s}\right)^{3} \\
& =\sum_{\substack{l_{\tilde{\alpha}} \mid\left(M / l_{1}\right) \\
\left(l_{\tilde{\alpha}}, 2\right)=1}} \chi_{a_{1} d_{0} l_{1}}\left(l_{\tilde{\alpha}}\right) l_{\tilde{\alpha}}^{-1+s} \cdot \sum_{\substack{l_{\tilde{\beta}}\left(M / l_{1}\right) \\
\left(l_{\tilde{\beta}}, 2\right)=1}} \chi_{a_{1} d_{0} l_{1}}\left(l_{\tilde{\beta}}\right) l_{\tilde{\beta}}^{-1+s} \times \\
& \quad \times \sum_{\substack{l_{\tilde{\gamma}} \mid\left(M / l_{1}\right) \\
(\tilde{\gamma}, 2)=1}} \chi_{a_{1} d_{0} l_{1}}\left(l_{\tilde{\gamma}}\right) l_{\tilde{\gamma}}^{-1+s} .
\end{aligned}
$$

It is quite clear that (4.22) decomposes into a linear combination of the functions

$$
Z_{M}\left(1-s, w+3 s-3 / 2 ; \chi^{(*)}, \chi_{a_{1} l_{1}}\right)
$$


depending upon the congruence class of $a_{1} l_{1}$ modulo 4 . Since the shape of the final result is very similar in all the three cases (as in the previous proposition, just the behavior at the finite place 2 changes), we will just consider the case of $a_{1} l_{1} \equiv-1(\bmod 4)$, say. The character $\chi^{*}$ takes one of the two forms $\chi_{l_{\alpha} l_{\beta} l_{\gamma} l_{\bar{\alpha}} l_{\beta} l_{\bar{\gamma}}} \chi_{a_{2} l_{2}}$, $\chi_{-1} \chi_{l_{\alpha} l_{\beta} l_{l} l_{\bar{l}} l_{\beta} l_{3}} \chi_{a_{2} l_{2}}$. Note that for $d_{0} \equiv 1(\bmod 4), \chi_{a_{1} d_{0} l_{1}}(2)=0$ and $\chi_{a_{1} d_{0} l_{1}}\left(l^{\prime}\right)=$ $\chi_{a_{1} l_{1}}\left(l^{\prime}\right) \chi_{d_{0}}\left(l^{\prime}\right)=\chi_{a_{1} l_{1}}\left(l^{\prime}\right) \chi_{l^{\prime}}\left(d_{0}\right)$, for $\left(l^{\prime}, 2\right)=1$. For $d_{0} \equiv-1(\bmod 4)$ and any $l>0, \chi_{a_{1} d_{0} l_{1}}(l)=\chi_{l}\left(a_{1} l_{1}\right) \chi_{l}\left(d_{0}\right)$. Using this and the character $\chi_{-1}$ to separate the congruence classes, $1,-1(\bmod 4)$, we combine (4.22) with the definition of $Z_{M}$ in (4.13) to obtain

$$
\begin{aligned}
& Z_{M}\left(s, w ; \chi_{a_{2} l_{2}}, \chi_{a_{1} l_{1}}\right) \cdot \prod_{p \mid\left(M / l_{1}\right)}\left(1-p^{-2+2 s}\right)^{3} \\
& =l_{1}^{3 / 2-3 s} \frac{G_{\epsilon\left(a_{1}\right)}(1-s)^{3}}{G_{\epsilon\left(a_{1}\right)}(s)^{3}} \cdot \frac{1}{2}\left[4^{3 / 2-3 s}\left(1-2^{-2+2 s}\right)^{3} \times\right. \\
& \times \sum_{l_{\alpha} \mid\left(M / l_{1}\right),\left(2, l_{\alpha}\right)=1} \mu\left(l_{\alpha}\right) \chi_{a_{1} l_{1}}\left(l_{\alpha}\right) l_{\alpha}^{-s} \cdot \sum_{l_{\beta} \mid\left(M / l_{1}\right),\left(2, l_{\beta}\right)=1} \mu\left(l_{\beta}\right) \chi_{a_{1} l_{1}}\left(l_{\beta}\right) l_{\beta}^{-s} \times \\
& \times \sum_{l_{y} \mid\left(M / l_{1}\right),\left(2, l_{\gamma}\right)=1} \mu(l \gamma) \chi_{a_{1} l_{1}}\left(l_{\gamma}\right) l_{\gamma}^{-s} \cdot \sum_{l_{\tilde{\alpha}} \mid\left(M / l_{1}\right),\left(2, l_{\tilde{\alpha}}\right)=1} \chi_{a_{1} l_{1}}\left(l_{\tilde{\alpha}}\right) l_{\tilde{\alpha}}^{-1+s} \times \\
& \times \sum_{l_{\tilde{\beta}} \mid\left(M / l_{1}\right),\left(2, l_{\tilde{\beta}}\right)=1} \chi_{a_{1} l_{1}}\left(l_{\tilde{\beta}}\right) l_{\tilde{\beta}}^{-1+s} \cdot \sum_{l_{\tilde{\gamma}} \mid\left(M / l_{1}\right),\left(2, l_{\tilde{\gamma}}\right)=1} \chi_{a_{1} l_{1}}\left(l_{\tilde{\gamma}}\right) l_{\tilde{\gamma}}^{-1+s} \times \\
& \times\left(Z_{M}\left(1-s, w+3 s-3 / 2 ; \chi_{l_{\alpha} l_{\beta} l_{\gamma} l_{\bar{\alpha}} l_{\bar{\beta}} l_{\bar{\gamma}}} \chi_{a_{2} l_{2}}, \chi_{a_{1} l_{1}}\right)+\right. \\
& \left.+Z_{M}\left(1-s, w+3 s-3 / 2 ; \chi_{-1} \chi_{l_{\alpha} l_{\beta} l_{\gamma} l_{\alpha} l_{\bar{\beta}} l_{\bar{\gamma}}} \chi_{a_{2} l_{2}}, \chi_{a_{1} l_{1}}\right)\right)+ \\
& +\sum_{l_{\alpha} \mid\left(M / l_{1}\right)} \mu\left(l_{\alpha}\right) \chi_{l_{\alpha}}\left(a_{1} l_{1}\right) l_{\alpha}^{-s} \cdot \sum_{l_{\beta} \mid\left(M / l_{1}\right)} \mu\left(l_{\beta}\right) \chi_{l_{\beta}}\left(a_{1} l_{1}\right) l_{\beta}^{-s} \cdot \sum_{l_{\gamma} \mid\left(M / l_{1}\right)} \mu\left(l_{\gamma}\right) \chi_{l_{\gamma}}\left(a_{1} l_{1}\right) l_{\gamma}^{-s} \times \\
& \times \sum_{l_{\bar{\alpha}} \mid\left(M / l_{1}\right)} \chi_{l_{\tilde{\alpha}}}\left(a_{1} l_{1}\right) l_{\tilde{\alpha}}^{-1+s} \cdot \sum_{l_{\tilde{\beta}} \mid\left(M / l_{1}\right)} \chi_{l_{\tilde{\beta}}}\left(a_{1} l_{1}\right) l_{\tilde{\beta}}^{-1+s} \cdot \sum_{l_{\bar{\gamma}} \mid\left(M / l_{1}\right)} \chi_{l_{\bar{\gamma}}}\left(a_{1} l_{1}\right) l_{\tilde{\gamma}}^{-1+s} \times \\
& \times\left(Z_{M}\left(1-s, w+3 s-3 / 2 ; \chi_{l_{\alpha} l_{\beta} l_{\gamma} l_{\bar{\alpha}} l_{\bar{\beta}} l_{\gamma}} \chi_{a_{2} l_{2}}, \chi_{a_{1} l_{1}}\right)-\right.
\end{aligned}
$$

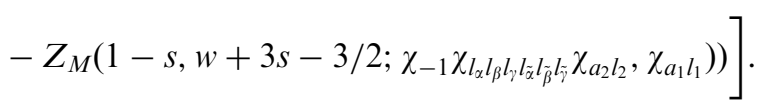

This rather complicated formula is the content of Proposition 4.3, where it is expressed in a considerably more compact way.

This completes the proof of Proposition 4.3.

\subsection{THE ANALYTIC CONTINUATION OF $Z_{M}\left(s, w ; \chi_{a_{2} l_{2}}, \chi_{a_{1} l_{1}}\right)$}

We begin by recalling some fundamental concepts from the theory of several complex variables. Our basic reference is Hörmander [Hö]. 
DEFINITION 4.4. An open set $R$ in $\mathrm{C}^{m}$ is called a domain of holomorphy if there are no open sets $R_{1}$ and $R_{2}$ in $\mathrm{C}^{m}$ such that $\varnothing \neq R_{1} \subset R_{2} \cap R, R_{2}$ is connected and not contained in $R$, and for any holomorphic function $f$ in $R$ there exists a holomorphic function $f_{2}$ in $R_{2}$ satisfying $f=f_{2}$ in $R_{1}$.

DEFINITION 4.5. An open set $\Omega$ in $\mathbb{C}^{m}$ is called a tube if there is an open set $\omega$ in $\mathbb{R}^{m}$, called the base of $\Omega$, such that $\Omega=\{s \mid \Re(s) \in \omega\}$.

We will denote by $\hat{R}$, the convex hull of a subset $R \subset \mathbb{R}^{m}$ or $\mathbb{C}^{m}$. It is easy to see that the convex hull $\hat{\Omega}$ of a tube $\Omega$ is a tube with base $\hat{\omega}$.

PROPOSITION 4.6. If $\Omega$ is a connected tube, then any holomorphic function in $\Omega$ can be extended to a holomorphic function $\hat{f}$ in $\hat{\Omega}$.

PROPOSITION 4.7. Let $R$ and $R^{\prime}$ be domains of holomorphy in $\mathrm{C}^{m}$ and $\mathbb{C}^{n}$, respectively, and let $f$ be an analytic map of $R$ into $\mathbb{C}^{n}$. Then the set

$$
R_{f}=\left\{s \in R \mid f(s) \in R^{\prime}\right\}
$$

is a domain of holomorphy.

In order to analytically continue $Z_{M}\left(s, w ; \chi_{a_{2} l_{2}}, \chi_{a_{1} l_{1}}\right)$ as a function of two complex variables $s, w$, we repeatedly apply the functional equations given in Propositions 4.2, 4.3.

Accordingly, we define two involutions on $\mathrm{C} \times \mathrm{C}$ :

$$
\alpha:(s, w) \rightarrow(1-s, w+3 s-3 / 2) \quad \text { and } \quad \beta:(s, w) \rightarrow(s+w-1 / 2,1-w) .
$$

Then $a, \beta$ generate $D_{12}$, the dihedral group of order 12 , and $\alpha^{2}=\beta^{2}=1$, $(\alpha \beta)^{6}=(\beta \alpha)^{6}=1$. Note that $\alpha \beta \neq \beta \alpha$.

We will find it useful in the following to define three regions $R_{1}, R_{2}, R_{3}$ as follows: Write $s, w$ as $s=\sigma+i t, w=v+i \gamma$.

The tube region $R_{1}$ is defined to be the set of all points $(s, w)$ such that $(\sigma, v)$ lie strictly above the polygon determined by $(0,5 / 2),(3 / 2,0)$, and the rays $v=-3 \sigma+5 / 2$ for $\sigma \leqslant 0$ and $v=-\sigma+3 / 2$ for $\sigma \geqslant 3 / 2$. Note that $R_{1}$ is the convex closure of the region given in Figure 1 which is bounded by the dotted lines and the two rays $v=-3 \sigma+5 / 2$ for $\sigma \leqslant 0$ and $v=-\sigma+3 / 2$ for $\sigma \geqslant 3 / 2$, which is the actual region that comes up in the proof of Propositions 4.8, 4.9.

The tube region $R_{2}$ is defined to be the set of all points $(s, w)$ such that $(\sigma, v)$ lie strictly above the line segment connecting $(-1 / 2,3)$ and $(3 / 2,0)$ and the rays $v=-2 \sigma+2$ for $\sigma \leqslant-1 / 2$, and $v=-\sigma+3 / 2$ for $\sigma \geqslant 3 / 2$.

The tube region $R_{3}$ is defined to be the set of all points $(s, w)$ such that $(\sigma, v)$ lie strictly above the line $v=-2 \sigma+2$.

These regions are related by the involutions $\alpha, \beta$ as described in the following proposition. The proof, a simple exercise, is omitted. 
PROPOSITION 4.8. The regions $R_{1}$ and $\alpha\left(R_{1}\right)$ have a nonempty intersection, and the convex hull of $R_{1} \cup \alpha\left(R_{1}\right)$ equals $R_{2}$. Similarly, $R_{2}$ and $\beta\left(R_{2}\right)$ have a nonempty intersection and the convex hull of $R_{2} \cup \beta\left(R_{2}\right)$ equals $R_{3}$. Finally, $R_{3}$ and $\alpha\left(R_{3}\right)$ have a nonempty intersection and the convex hull of $R_{3} \cup \alpha\left(R_{3}\right)$ equals $\mathbb{C}^{2}$.

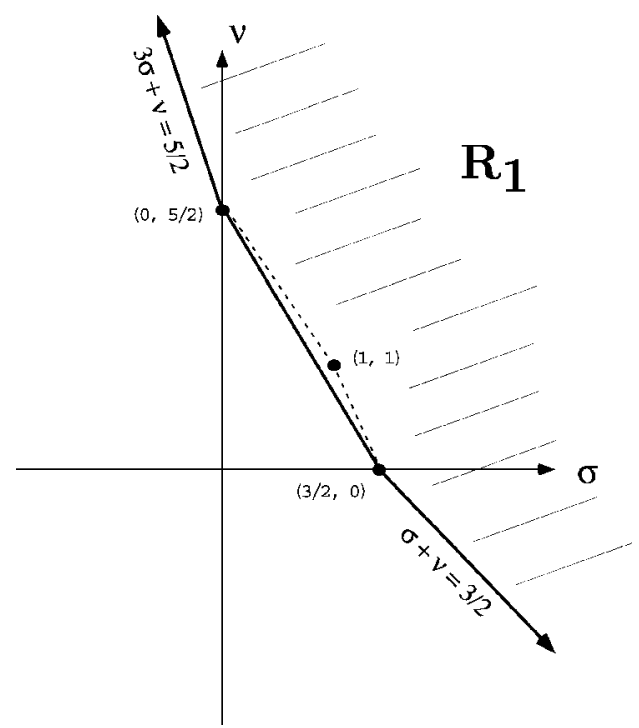

Figure 1 .

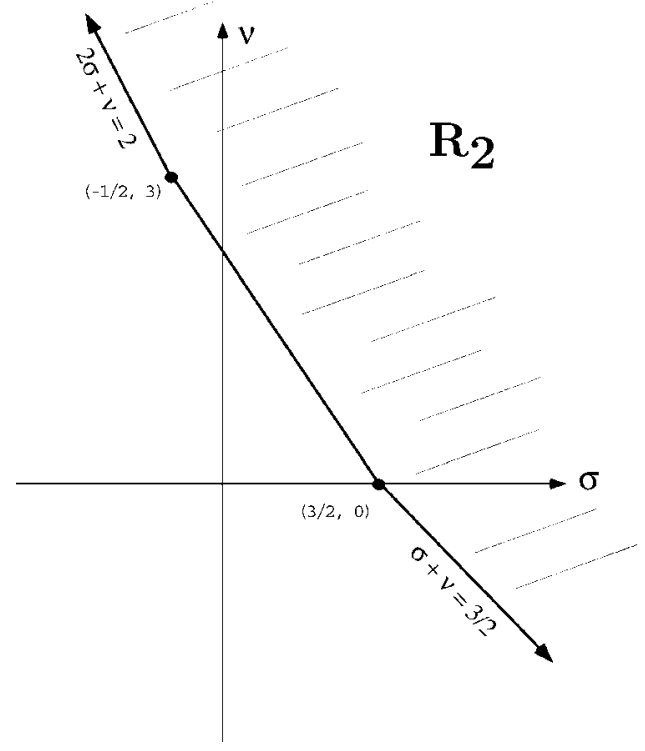

Figure 2. 


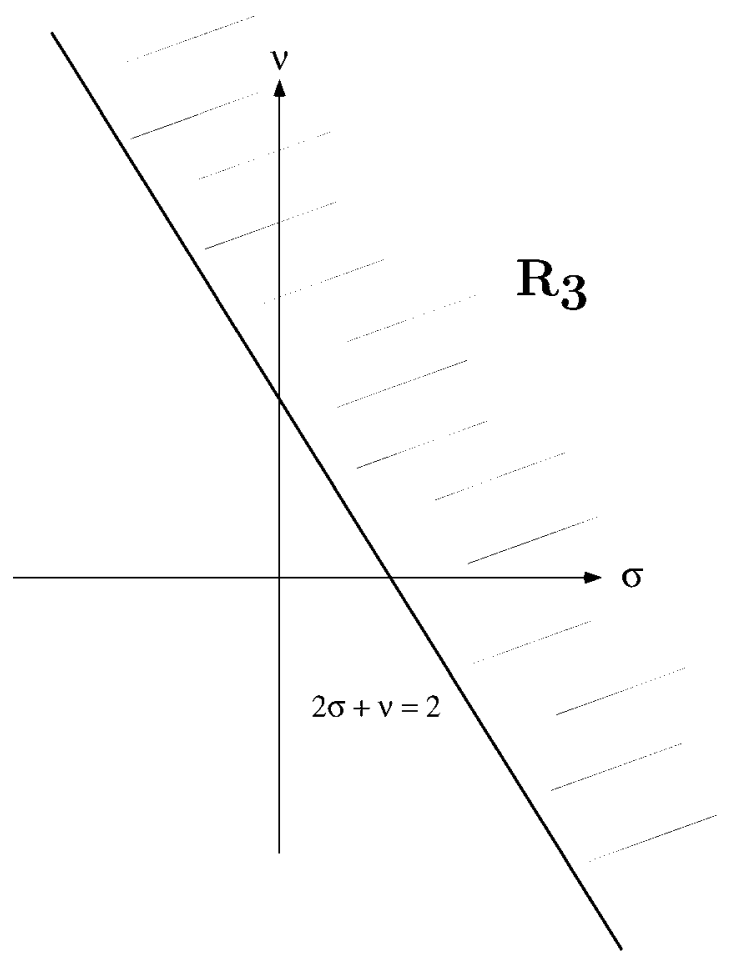

Figure 3.

Let

$$
P(s, w)=(s-1)^{3}(w-1) .
$$

We will begin by demonstrating the following proposition:

PROPOSITION 4.9. Let $R_{1}$ be the tube region defined above. The function

$$
P(s, w) Z_{M}\left(s, w ; \chi_{a_{2} l_{2}}, \chi_{a_{1} l_{1}}\right)
$$

is analytic in $R_{1}$.

Proof. Consider first the left-hand side of the expression for $Z_{M}\left(s, w ; \chi_{a_{2} l_{2}}, \chi_{a_{1} l_{1}}\right)$ given in (4.10). If the sum were restricted only to square free $d=d_{0}$, then the usual Phragmen-Lindelöf bounds or $L\left(s, \chi_{d_{0}}\right)$ would imply absolute convergence for $v>1$ when $\sigma>1$, for $v>(-3 / 2) \sigma+5 / 2$ when $0 \leqslant \sigma \leqslant 1$ and for $v>-3 \sigma+5 / 2$ when $\sigma<0$. Because we have the bound (4.11) and functional equation (4.7) applied to $P_{d_{0}, d_{1}}^{\left(a_{1}, l_{1}\right)}(s)$, precisely the same estimates apply as we sum over all $d$. Consequently, $Z_{M}\left(s, w ; \chi_{a_{2}, l_{2}}, \chi_{a_{1}, l_{1}}\right)$ converges above the given lines, and the factor $(s-1)^{3}$ in $P(s, w)$ cancels the pole at $s=1$.

Noting that both sides of the expression converge when $v, \sigma>1$, we now change the order of summation and examine the right-hand side. Here the coefficients $c(n)$ 
are order 3 divisor functions and are bounded above by $n^{c}$ for any $\epsilon>0$. Consequently, applying Phragmen-Lindelöf again to $L\left(w, \chi_{n_{0}}\right)$ and the corresponding estimate and functional equations for $c\left(n_{0} n_{1}^{2}\right) Q_{n_{0}, n_{1}}^{\left(a_{2} l_{2}\right)}(w)$, we obtain convergence of $Z_{M}\left(s, w ; \chi_{a_{2} l_{2}}, \chi_{a_{1} l_{1}}\right)$ for $\sigma>1$ when $v>1$, for $\sigma>(-1 / 2) v+3 / 2$ when $0 \leqslant v \leqslant 1$ and $\sigma>-v+3 / 2$ when $v<0$. The factor $w-1$ in $P(s, w)$ cancels the pole at $w=1$. These regions overlap when $v, \sigma>1$, and thus by Proposition 4.6, $Z_{M}\left(s, w ; \chi_{a_{2} l_{2}}, \chi_{a_{1} l_{1}}\right) P(s, w)$ has an analytic continuation to the convex closure of the regions, which is $R_{1}$ described above.

This completes the proof of Proposition 4.14.

Our plan is now to apply the involutions $\alpha, \beta, \alpha$ in that order to $R_{1}$, and use Propositions 4.2 and 4.3 to extend the analytic continuation to $\mathbb{C}^{2}$. To aid in this, it will be useful to introduce some additional notation to make the content of these propositions a bit clearer and easier to apply. Let

$$
A(s, w) \equiv A_{M}(s, w)=\prod_{p \mid M}\left(1-p^{-2+2 s}\right)^{3}
$$

and

$$
B(s, w) \equiv B_{M}(s, w)=\prod_{p \mid M}\left(1-p^{-2+2 w}\right),
$$

and let $\quad \tilde{\Psi}^{\left(a_{1} l_{1}\right)}(s, w)=\Psi^{\left(a_{1} l_{1}\right)}(s) \prod_{p \mid l_{1}}\left(1-p^{-2+2 s}\right)^{3}, \tilde{\Phi}^{\left(a_{2} l_{2}\right)}(s, w)=\Phi^{\left(a_{2} l_{2}\right)}(w) \prod_{p \mid l_{2}}(1-$ $\left.p^{-2+2 w}\right)$.

The following is a reformulation of the content we require now from Propositions 4.2 and 4.3. For $(s, w)$ such that both sides are contained in a connected region of analytic continuation for $P(s, w) Z_{M}\left(s, w ; \chi_{a_{2} l_{2}}, \chi_{a_{1}, l_{1}}\right)$

$$
A(s, w) \vec{Z}_{M}\left(s, w ; \chi_{\operatorname{Div}(\mathrm{M})}, \chi_{a_{1} l_{1}}\right)=\tilde{\Psi}^{\left(a_{1} l_{1}\right)}(s, w) \vec{Z}_{M}\left(\alpha(s, w) ; \chi_{\operatorname{Div}(\mathrm{M})}, \chi_{a_{1} l_{1}}\right)
$$

and

$$
\begin{aligned}
& B(s, w) \vec{Z}_{M}\left(s, w ; \chi_{a_{2} l_{2}}, \chi_{\operatorname{Div}(\mathrm{M})}\right) \\
& \quad=\tilde{\Phi}^{\left(a_{2} l_{2}\right)}(s, w) \vec{Z}_{M}\left(\beta(s, w) ; \chi_{a_{2} l_{2}}, \chi_{\operatorname{Div}(\mathrm{M})}\right) .
\end{aligned}
$$

The following proposition will now complete the analytic continuation of $Z_{M}\left(s, w ; \chi_{a_{2} l_{2}}, \chi_{a_{1} l_{1}}\right)$.

PROPOSITION 4.10. Let

$$
\begin{aligned}
\mathcal{P}(s, w)= & s^{3}(s-1)^{3}(s+w-3 / 2)^{3}(2 s+w-1)^{3}(s+w-1 / 2)^{3}(2 s+w-2)^{3} \times \\
& \times w(w-1)(3 s+w-5 / 2)(3 s+2 w-3)(3 s+w-3 / 2) .
\end{aligned}
$$


Then the following product has an analytic continuation to an entire function in $\mathrm{C}^{2}$ :

$$
\begin{aligned}
\tilde{Z}_{M}\left(s, w ; \chi_{a_{2} l_{2}}, \chi_{a_{1} l_{1}}\right):= & A(s, w) A(\alpha(s, w)) A(\beta(s, w)) A(\beta \alpha(s, w)) B(s, w) \times \\
& \times B(\alpha(s, w)) \mathcal{P}(s, w) Z_{M}\left(s, w ; \chi_{a_{2} l_{2}}, \chi_{a_{1} l_{1}}\right) .
\end{aligned}
$$

Proof. In Proposition 4.9 we established the continuation of $Z_{M}\left(s, w ; \chi_{a_{2} l_{2}}, \chi_{a_{1} l_{1}}\right)$ $P(s, w)$ in $R_{1}$. As $\alpha^{2}=1$ and $\tilde{\Psi}^{\left(a_{1} l_{1}\right)}(s, w)$ is meromorphic in $\mathbb{C}^{2}$, it follows that

$$
\tilde{\Psi}^{\left(a_{1} l_{1}\right)}(s, w) \vec{Z}_{M}\left(\alpha(s, w) ; \chi_{\operatorname{Div}(\mathrm{M})}, \chi_{a_{1}, l_{1}}\right) P(\alpha(s, w))
$$

is a meromorphic function in $\alpha\left(R_{1}\right)$. From (4.23), we observe that poles can just occur at the points $s=1,3,5, \ldots$ or $s=2,4,6, \ldots$ (depending on $\epsilon\left(a_{1}\right)$ ). However, except for the possible pole at $s=1$, all the others are canceled by the trivial zeros of $L\left(1-s, \chi_{d_{0}}\right)$. We can conclude from Proposition 4.9 and (4.26) that $A(s, w) P(s, w) P(\alpha(s, w)) Z{ }^{\rightarrow}{ }_{M}\left(s, w ; \chi_{\operatorname{Div}(\mathrm{M})}, \chi_{a_{1} l_{1}}\right)$ is analytic in $R_{1} \cup \alpha\left(R_{1}\right), R_{1}$ and $\alpha\left(R_{1}\right)$ having a substantial intersection (containing $\Re(s), \mathfrak{R}(w)>1$ ). Thus by Proposition 4.6, this function is analytic in $R_{2}$, the convex hull of the union.

Since $\beta^{2}=1$ and $\tilde{\Phi}^{\left(a_{2} l_{2}\right)}(s, w)$ is moromorphic in $\mathbb{C}^{2}$, it follows from what we have just proved that

$$
\tilde{\Phi}^{\left(a_{2} l_{2}\right)}(s, w) A(\beta(s, w)) P(\beta(s, w)) P(\alpha \beta(s, w)) \vec{Z}_{M}\left(\beta(s, w) ; \chi_{a_{2} l_{2}}, \chi_{\operatorname{Div}(\mathrm{M})}\right)
$$

is a meromorphic function in $\beta\left(R_{2}\right)$. As before, all the poles, except the possible one at $w=1$, of $\tilde{\Phi}^{\left(a_{2} l_{2}\right)}(s, w)$ are canceled by trivial zeros of $L$-functions. From (4.27), we conclude that

$$
A(s, w) A(\beta(s, w)) B(s, w) P(s, w) P(\alpha(s, w)) P(\beta(s, w)) P(\alpha \beta(s, w)) \vec{Z}_{M}\left(s, w ; \chi_{a_{2} l_{2}}, \chi_{\operatorname{Div}(M)}\right)
$$

is an analytic function in $R_{2} \cup \beta\left(R_{2}\right)$. As this has a non-empty intersection, it follows from Proposition 4.6 again that (4.28) is analytic in $R_{3}$, the convex hull of $R_{2} \cup \beta\left(R_{2}\right)$.

To complete the argument, apply $\alpha$ to (4.26), obtaining

$$
A(\alpha(s, w)) \vec{Z}_{M}\left(\alpha(s, w) ; \chi_{\operatorname{Div}(\mathrm{M})}, \chi_{a_{1} l_{1}}\right)=\tilde{\Psi}^{\left(a_{1} l_{1}\right)}(\alpha(s, w)) \vec{Z}_{M}\left(s, w ; \chi_{\operatorname{Div}(\mathrm{M})}, \chi_{a_{1} l_{1}}\right) .
$$

Multiplying the above by $A(s, w) A(\beta(s, w)) B(s, w) P(s, w) P(\alpha(s, w)) P(\beta(s, w))$ $P(\alpha \beta(s, w))$ and applying (4.28), we see that

$$
\begin{aligned}
& A(s, w) A(\alpha(s, w)) A(\beta(s, w)) B(s, w) P(s, w) P(\alpha(s, w)) P(\beta(s, w)) \times \\
& \quad \times P(\alpha \beta(s, w)) \vec{Z}_{M}\left(\alpha(s, w) ; \chi_{\operatorname{Div}(\mathrm{M})}, \chi_{a_{1} l_{1}}\right)
\end{aligned}
$$


is analytic for $(s, w) \in R_{3}$. Replacing $(s, w)$ by $\alpha(s, w)$, we obtain

$$
\begin{aligned}
& A(s, w) A(\alpha(s, w)) A(\beta(s, w)) A(\beta \alpha(s, w)) B(s, w) B(\alpha(s, w)) P(s, w) P(\alpha(s, w)) \times \\
& \quad \times P(\beta(s, w)) P(\beta \alpha(s, w)) P(\alpha \beta(s, w)) P(\alpha \beta \alpha(s, w)) \vec{Z}_{M}\left(s, w ; \chi_{\operatorname{Div}(\mathrm{M})}, \chi_{a_{1} l_{1}}\right)
\end{aligned}
$$

is analytic for $(s, w) \in \alpha\left(R_{3}\right)$. Combining this with the fact that (4.28) is analytic in $R_{3}$, we obtain the analytic of

$$
\begin{aligned}
& A(s, w) A(\alpha(s, w)) A(\beta(s, w)) A(\beta \alpha(s, w)) B(s, w) B(\alpha(s, w)) P(s, w) P(\alpha(s, w)) \times \\
& \quad \times P(\beta(s, w)) P(\beta \alpha(s, w)) P(\alpha \beta(s, w)) P(\alpha \beta \alpha(s, w)) \vec{Z}_{M}\left(s, w ; \chi_{\operatorname{Div}(\mathrm{M})}, \chi_{a_{1} l_{1}}\right)
\end{aligned}
$$

in $R_{3} \cup \alpha\left(R_{3}\right)$. As this has a nonempty intersection, it follows from Proposition 4.6 again that the above is analytic in $\mathbb{C}^{2}$, the convex hull of $R_{3} \cup \beta\left(R_{3}\right)$.

In fact, $P(\alpha \beta(s, w)), P(\alpha \beta \alpha(s, w))$ have one factor in common: $2 w+3 s-3$, and so in the last step we included one unnecessary multiple of $2 w+3 s-3$. Removing this, we complete the proof of Proposition 4.10.

\subsection{AN ESTIMATE FOR $Z_{M}\left(\frac{1}{2}, w ; \chi_{a_{2} l_{2}}, \chi_{a_{1} l_{1}}\right)$ IN VERTICAL STRIPS}

In this section we will use the analytic continuation and functional equations (4.26), (4.27) for $\vec{Z}_{M}\left(s, w ; \chi_{\operatorname{Div}(\mathrm{M})}, \chi_{a_{1} l_{1}}\right)$ to locate poles and obtain an estimate for the growth of this function in a vertical strip. Before doing this, however, we need some additional notation.

Let $\vec{Z}_{M}(s, w)$ denote the $4 d(M)^{2}$-dimensional column vector consisting of the concatenation of the $2 d(M)$ column vectors $\vec{Z}_{M}\left(s, w ; \chi_{a_{2} l_{2}}, \chi_{\operatorname{Div}(\mathrm{M})}\right)$ for $a_{2} \in\{1,-1\}$ and all $l_{2} \mid M$. Then by Propositions 4.2 and 4.3, combined with (4.26), (4.27), there exist $4 d(M)^{2}$ by $4 d(M)^{2}$ matrices $\Phi_{M}(s, w), \Psi_{M}(s, w)$ such that

$$
A_{M}(s, w) \vec{Z}_{M}(s, w)=\Psi_{M}(s, w) \vec{Z}_{M}(\alpha(s, w))
$$

and

$$
B_{M}(s, w) \vec{Z}_{M}(s, w)=\Phi_{M}(s, w) \vec{Z}_{M}(\beta(s, w))
$$

Here $A_{M}(s, w), B_{M}(s, w)$ are given by (4.25). The matrices $\Phi_{M}(s, w), \Psi_{M}(s, w)$ are constructed from blocks of $\tilde{\Phi}^{\left(a_{2} l_{2}\right)}(s, w)$ and $\tilde{\Psi}^{\left(a_{1} l_{1}\right)}(s, w)$ on the diagonal.

Next, we use Proposition 4.7 to show that the function $\tilde{Z}_{M}\left(1 / 2, w ; \chi_{a_{2} l_{2}}, \chi_{a_{1} l_{1}}\right)$, defined in Proposition 4.10, is of finite order. Although it seems to be a one-variable problem, the theory of several complex variables is still needed in the proof.

PROPOSITION 4.11. The entire function

$$
\tilde{Z}_{M}\left(\frac{1}{2}, w ; \chi_{a_{2} l_{2}}, \chi_{a_{1} l_{1}}\right)
$$

is of the first order. 
Proof. First, the convexity bound $L\left(1 / 2, \chi_{d_{0}}\right) \ll_{\epsilon} d_{0}^{\frac{1}{4}+\epsilon}$ together with (4.11), implies that

$$
Z_{M}\left(\frac{1}{2}, w ; \chi_{a_{2} l_{2}}, \chi_{a_{1} l_{1}}\right) \ll_{\epsilon} 1
$$

for $\mathfrak{R}(w)=v>\frac{7}{4}+\epsilon$. Applying (4.29) and (4.30) several times in succession, we obtain

$$
\begin{aligned}
\vec{Z}_{M}(s, w)= & B_{M}(s, w)^{-1} \Phi_{M}(s, w) A_{M}(\beta(s, w))^{-1} \Psi_{M}(\beta(s, w)) B_{M}(\alpha \beta(s, w))^{-1} \times \\
& \times \Phi_{M}(\alpha \beta(s, w)) A_{M}(\beta \alpha \beta(s, w))^{-1} \Psi_{M}(\beta \alpha \beta(s, w)) B_{M}\left((\alpha \beta)^{2}(s, w)\right)^{-1} \times \\
& \times \Phi_{M}\left((\alpha \beta)^{2}(s, w)\right) \vec{Z}_{M}(s, 5 / 2-3 s-w) .
\end{aligned}
$$

For $s=1$, we observe that $\vec{Z}_{M}(1 / 2, w)$ is related to $\vec{Z}_{M}(1 / 2,1-w)$ by the functional equation (4.31). Using Stirling's formula, we can bound from above the entries of the right hand side matrices in (4.31), obtaining

$$
Z_{M}\left(\frac{1}{2}, v+i t ; \chi_{a_{2} l_{2}}, \chi_{a_{1} l_{1}}\right) \ll_{\epsilon}(1+|t|)^{C},
$$

where $C$ is an absolute positive constant and $v<-\frac{3}{4}-\epsilon$.

The proof of Proposition 4.11 is based on an application of Proposition 4.7 to the function $f: \mathbb{C}^{2} \rightarrow \mathbb{C}$, defined by

$$
f(s, w)=\Gamma(s+5) \Gamma(w+5) \tilde{Z}_{M}\left(s, w ; \chi_{a_{2} l_{2}}, \chi_{a_{1} l_{1}}\right) .
$$

Now let $\Omega_{0}$ be the tube region whose base is given in Figure 1. This tube already appeared at the end of the proof of Proposition 4.9 (its convex hull is $R_{1}$ ). Reflecting several times under $\alpha, \beta, \alpha, \beta \ldots$, until it stabilizes and then taking the union, we obtain a tube whose base is $\mathbb{R}^{2}$ with a hole in the middle (see Figure 4 below).

This hole is a tube with base a polygon, which lies inside the open ball $B(0,4)$ (of radius 4 centered at the origin) in $\mathbb{R}^{2}$. The function $\tilde{Z}_{M}\left(s, w ; \chi_{a_{2} l_{2}}, \chi_{a_{1} l_{1}}\right)$ is obviously of polynomial growth in $\Im(s)$ and $\Im(w)$ as long as $(s, w) \in \Omega_{0}$, and $\sigma, v$ are both bounded. Applying Stirling's formula in equations (4.18) and (4.23), we observe that the same holds when $\alpha, \beta$ are applied. Combining this with Stirling's formula, we conclude that the function $f(s, w)$ is bounded in the tube $\Omega^{\prime}$ with base the annulus $\omega^{\prime}=\left\{(\sigma, v) \in \mathbb{R}^{2} \mid 16<\sigma^{2}+v^{2}<25\right\}$. See Figure 5 below.

Let $R \subset \mathbb{C}^{2}$ be the tube whose base is $B(0,5)$ in $\mathbb{R}^{2}$, and let $R^{\prime}=B(0, m) \in \mathbb{C}$, where $m$ is an upper bound for $f$ on the annulus $\Omega^{\prime}$. Since $B(0,5)$ in $\mathbb{R}^{2}$ is a convex set, it follows that $R$ is a domain of holomorphy. Obviously, $R^{\prime}$ is also a domain of holomorphy. Applying Proposition 4.7, it follows that

$$
\Gamma(s+5) \Gamma(w+5) \tilde{Z}_{M}\left(s, w ; \chi_{a_{2} l_{2}}, \chi_{a_{1} l_{1}}\right)
$$




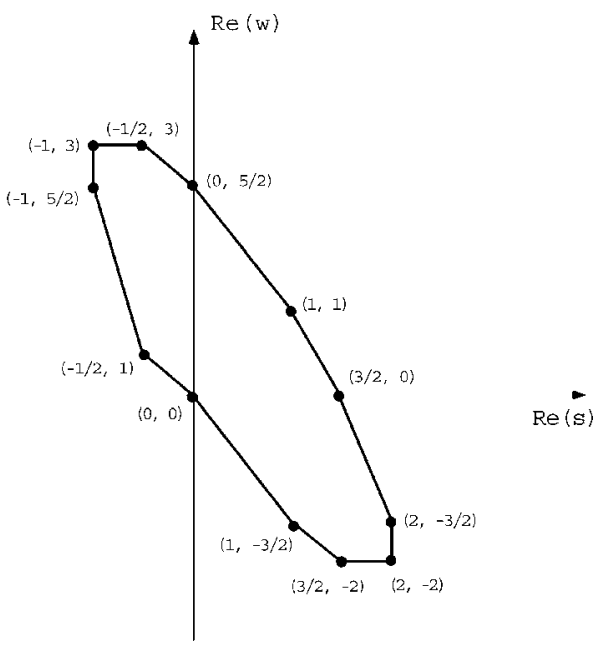

Figure 4.

is bounded in $R$, since in this case, the set $R_{f}$ contains the annulus $\Omega^{\prime}$ whose convex closure contains $R$. In particular, the function is bounded in the tube with base given by the polygon in Figure 4. Proposition 4.11 immediately follows.

One of the key ingredients in what follows, is that the series

$$
\sum_{d_{0}}\left|L\left(\frac{1}{2}+i t, \chi_{d_{0}}\right)\right|^{4}\left|d_{0}\right|^{-v}
$$

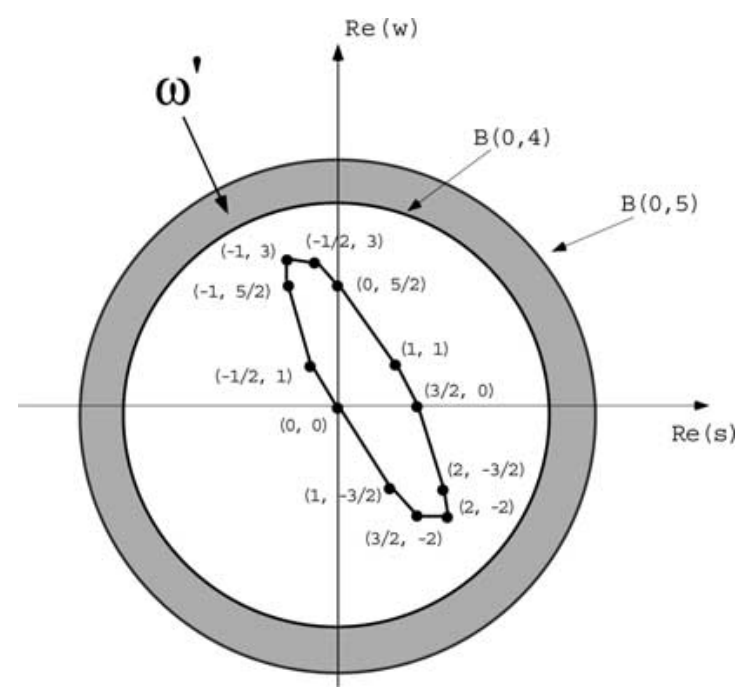

Figure 5 . 
is convergent, for $v=\Re(w)>1$. Here the summation is over all positive or negative square free integers. This follows from the work of Heath-Brown [H-B]. Applying the Cauchy-Schwartz inequality, we deduce that

$$
\sum_{d}\left|c_{d}\right|\left|L\left(\frac{1}{2}+i t, \chi_{d}\right)\right|^{3}|d|^{-v}
$$

is convergent, for $v=\Re(w)>1$, and any sequence $c_{d}$ such that $c_{d} \ll_{\epsilon} d^{\epsilon}$. Here the summation is over all intergers.

We now show:

PROPOSITION 4.12. Let $w=v+i t$. For $\epsilon>0,-\epsilon \leqslant v$, and any $a_{1}, a_{2} \in\{1,-1\}$, $l_{1}, l_{2} \mid M$ the function $Z_{M}\left(1 / 2, w ; \chi_{a_{2} l_{2}}, \chi_{a_{1} l_{1}}\right)$ is an analytic function of $w$, except for possible poles at $w=\frac{3}{4}$ and $w=1$. If $\left(l_{1}, l_{2}\right)=1$ or 2 and $|t|>1$, then it satisfies the upper bounds

$$
Z_{M}\left(\frac{1}{2}, v+i t ; \chi_{a_{2} l_{2}}, \chi_{a_{1} l_{1}}\right) \ll_{\epsilon} 1,
$$

for $1+\epsilon<v$, and

$$
Z_{M}\left(\frac{1}{2}, v+i t ; \chi_{a_{2} l_{2}}, \chi_{a_{1} l_{1}}\right) \ll_{\epsilon} M^{3(1-v)+v_{1}(\epsilon)}|t|^{5(1-v)+v_{2}(\epsilon)} \sum_{a=1,-1} \sum_{l \mid M} \sum_{\left(d_{0}, M\right)=1} \frac{\left|L\left(\frac{1}{2}, \chi_{d_{0}} \chi_{a l}\right)\right|^{3}}{d_{0}^{1+\epsilon}}
$$

for $-\epsilon \leqslant v \leqslant 1+\epsilon$. The functions $v_{1}(\epsilon), v_{2}(\epsilon)$ are some explicitly computable functions satisfying

$$
\lim _{\epsilon \rightarrow 0} v_{1}(\epsilon)=\lim _{\epsilon \rightarrow 0} v_{2}(\epsilon)=0 .
$$

Proof. The first bound in the region $1+\epsilon<v$ is immediate by the remarks concerning (4.33). The bound for $-\epsilon \leqslant v \leqslant 1+\epsilon$ is more difficult to obtain. We shall first obtain a bound for $Z_{M}\left(\frac{1}{2}, v+\mathrm{it} ; \chi_{a_{2} l_{2}}, \chi_{a_{1} l_{1}}\right)$, (i.e., for $v=-\epsilon$ ), and then apply a convexity argument to complete the proof for $-\epsilon<v<1+\epsilon$.

Recall the functional equations

$$
\begin{aligned}
& \alpha(s, w)=\left(1-s, 3 s+w-\frac{3}{2}\right) \quad(\text { see Equation (4.23)), } \\
& \beta(s, w)=\left(s+w-\frac{1}{2}, 1-w\right) \quad(\text { see Equation (4.18)). }
\end{aligned}
$$

Fix $(s, w)=\left(\frac{1}{2},-\epsilon+i t\right)$. We then have

$$
\begin{aligned}
& \beta(s, w)=(-\epsilon+i t, 1+\epsilon-i t), \quad \alpha \beta(s, w)=(1+\epsilon-i t,-1 / 2-2 \epsilon+2 i t), \\
& \beta \alpha \beta(s, w)=(-\epsilon+i t, 3 / 2+2 \epsilon-2 i t), \quad \alpha \beta \alpha \beta(s, w)=(1+\epsilon-i t,-\epsilon+i t),
\end{aligned}
$$

and

$$
\beta \alpha \beta \alpha \beta(s, w)=\left(\frac{1}{2}, 1+\epsilon-i t\right) .
$$


We shall estimate $Z_{M}\left(\frac{1}{2}, v+i t ; \chi_{a_{2} l_{2}}, \chi_{a_{1} l_{1}}\right)$ by alternately applying the functional equations $\beta, \alpha$ as above. Note that each time we apply $\beta$ the value of $w$ is either $-\epsilon+$ it or $-\frac{1}{2}-2 \epsilon+2 i t$, and each time we apply $\alpha$, the value of $s$ is $-\epsilon+i t$. It is thus sufficient to obtain upper bounds in only these cases. We proceed to do this.

Now, it immediately follows from (4.18) and Stirling's asymptotic formula for the Gamma function that away from poles,

$$
\begin{aligned}
& Z_{M}\left(s,-\epsilon+i t ; \chi_{a_{2} l_{2}}, \chi_{a_{1} l_{1}}\right) \\
& \quad \ll_{\epsilon} \frac{1}{l_{2}^{2}+\epsilon} \sum_{l_{3}, l_{4} \mid M / l_{2}} M^{\epsilon} \sum_{a_{3}=1,-1}|t|^{\frac{1}{2}+\epsilon} \times \\
& \times\left(\left|Z_{m}\left(s-\frac{1}{2}-\epsilon+i t, 1+\epsilon-i t ; \chi_{a_{2} l_{2}}, \chi_{a_{1} l_{1} l_{3} l_{4}}\right)\right|+\right. \\
& \left.+\left|Z_{M}\left(s-\frac{1}{2}-\epsilon+i t, 1+\epsilon-i t ; \chi_{a_{2} l_{2}}, \chi_{-a_{1} l_{1} l_{3} l_{4}}\right)\right|\right) .
\end{aligned}
$$

Since $M$ is even and squarefree, we also have $\left(l_{2}, l_{1} l_{3} l_{4}\right)=1$ or 2 . The characters $\chi_{a_{1} l_{1} l_{3} l_{4}}$ and $\chi_{-a_{1} l_{1} l_{3} l_{4}}$ can be replaced by $\chi_{a_{1} d_{2}}, \chi_{-a_{1} d_{2}}$ with $d_{2}$ squarefree.

Similarly, for $w=-\frac{1}{2}-\epsilon+i t$, we have, after replacing $l_{2}$ by $d_{3}$ and $l_{1}$ by $d_{2}$ that

$$
\begin{aligned}
& Z_{M}\left(s,-\frac{1}{2}-2 \epsilon+2 i t ; \chi_{a_{2} d_{3}}, \chi_{a_{1} d_{2}}\right) \ll_{\epsilon} d_{3}^{1+\epsilon} \sum_{l_{3}, l_{4} \mid M / d_{3}} M^{\epsilon} l_{3}^{\frac{1}{2}} l_{4}^{-\frac{3}{2}} \sum_{a_{3}=1,-1}|t|^{1+\epsilon} \times \\
& \times\left(\left|Z_{m}\left(s-1-2 \epsilon+2 i t, \frac{3}{2}+2 \epsilon-2 i t ; \chi_{a_{2} d_{3}}, \chi_{a_{1} d_{4}}\right)\right|+\right. \\
& \left.\quad+\left|Z_{M}\left(s-1-2 \epsilon+2 i t, \frac{3}{2}+2 \epsilon-2 i t ; \chi_{a_{2} d_{3}}, \chi_{-a_{1} d_{4}}\right)\right|\right),
\end{aligned}
$$

where we have denoted by $d_{4}$, the squarefree part of $d_{2} l_{3} l_{4}$. Note that $\left(d_{3}, d_{4}\right)=1$ or 2 .

In a similar manner, we consider $s=-\epsilon+i t$ in (4.23). It follows from Stirling's formula that away from poles,

$$
\begin{aligned}
& Z_{M}\left(-\epsilon+i t, w ; \chi_{a_{2} l_{2}}, \chi_{a_{1} d_{2}}\right) \\
& \ll_{\epsilon}\left|d_{2} \cdot t\right|^{\frac{3}{2}+3 \epsilon} \sum_{l_{\alpha}, l_{\beta}, l_{y}, l_{\bar{\alpha}}, l_{\bar{\beta}}, l_{j} \mid\left(M / l_{1}\right)} M^{3 \epsilon} \times \\
& \times\left(\left|Z_{M}\left(1+\epsilon-i t, w-3 \epsilon+3 i t-\frac{3}{2} ; \chi_{l_{\alpha} l_{\beta} l_{\gamma}, l_{\alpha}, l_{\beta}, l_{\bar{\gamma}}} \cdot \chi_{a_{2} l_{2}}, \chi_{a_{1} d_{2}}\right)\right|+\right. \\
& \left.+\left|Z_{M}\left(1+\epsilon-i t, w-3 \epsilon+3 i t-\frac{3}{2} ; \chi-l_{\alpha} l_{\beta} l_{\gamma} l_{\bar{\alpha}}, l_{\bar{\beta}}, l_{\bar{\gamma}} \cdot \chi_{a_{2} l_{2}}, \chi_{a_{1}, d_{2}}\right)\right|\right) .
\end{aligned}
$$

As before, $\left(l_{\alpha} l_{\beta} l_{\gamma} l_{\bar{\alpha}} l_{\bar{\beta}} l_{\bar{\gamma}}, d_{2}\right)=1$ or 2 . We can replace $l_{\alpha} l_{\beta} l_{\gamma} l_{\bar{\alpha}} l_{\bar{\beta}} l_{\bar{\gamma}}$ by $d_{3}$, squarefree. We again obtain that $\left(d_{3}, d_{2}\right)=1$ or 2 . 
It now follows from the previous estimates and remarks that

$$
\begin{aligned}
& Z_{M}\left(\frac{1}{2},-\epsilon+i t ; \chi_{a_{2} l_{2}}, \chi_{a_{l_{1}} l_{1}}\right) \\
& \quad \ll_{\epsilon}|t|^{5+10 \epsilon} M^{10 \epsilon} d_{1}^{\frac{1}{2}+\epsilon} d_{2}^{\frac{3}{2}+3 \epsilon} d_{3}^{1+2 \epsilon} l_{3}^{\frac{1}{2}} l_{4}^{-\frac{3}{2}} d_{4}^{\frac{3}{2}+3 \epsilon} d_{5}^{\frac{1}{2}+\epsilon} \cdot S \\
& \quad=|t|^{5+10 \epsilon} M^{10 \epsilon}\left(d_{1} d_{2}\right)^{\frac{1}{2}+\epsilon} \times \\
& \quad \times\left(d_{2} d_{3}\right)^{\frac{1}{2}+\epsilon}\left(d_{3} d_{4}\right)^{\frac{1}{2}+\epsilon}\left(d_{4} d_{5}\right)^{\frac{1}{2}+\epsilon} d_{2}^{\frac{1}{2}+\epsilon} l_{3}^{\frac{1}{2}} l_{4}^{-\frac{3}{2}} d_{4}^{\frac{1}{2}+\epsilon} \cdot S,
\end{aligned}
$$

where $d_{1}=l_{2}, d_{j}=2^{\alpha_{j}} b_{j}, \alpha_{j}=0$ or 1 , and $\left.b_{j}\right|^{\frac{M}{2}},\left(b_{j}, b_{j+1}\right)=1(j=1,2, \ldots, 5)$, and $S$ is a sum of absolute values of the multiple Dirichlet series $Z_{M}$ at various arguments of the characters. We can take

$$
\begin{aligned}
S & =\sum_{a=1,-1} \sum_{l \mid M} Z_{M}\left(\frac{1}{2}, 1+\epsilon ; \chi_{a l}\right) \\
& =\sum_{a=1,-1} \sum_{l \mid M} \sum_{\substack{d=d_{0} d_{1}^{2} \\
(d, M)=1}} \frac{\left|L\left(\frac{1}{2}, F, \chi_{d_{0}} \chi_{a l}\right) P_{d_{0}, d_{1}}^{(a l)}(1 / 2)\right|}{d^{1+\epsilon}} .
\end{aligned}
$$

The positive integer $d_{4}$ is such that $d_{4}=d_{2} l_{3} l_{4}$ modulo squares, and $l_{3}, l_{4} \mid M$. Since $M$ is square free, it follows that

$$
\operatorname{ord}_{p}\left(\frac{d_{2} l_{3} d_{4}}{l_{4}^{3}}\right) \leqslant 2
$$

for any prime dividing $\frac{M}{2}$. Consequently,

$$
|t|^{5+10 \epsilon} M^{10 \epsilon}\left(d_{1} d_{2}\right)^{\frac{1}{2}+\epsilon}\left(d_{2} d_{3}\right)^{\frac{1}{2+\epsilon}}\left(d_{3} d_{4}\right)^{\frac{1}{2+\epsilon}}\left(d_{4} d_{5}\right)^{\frac{1}{2+\epsilon}} d_{2}^{\frac{1}{2}+\epsilon} l_{3}^{\frac{1}{2}} l_{4}^{-\frac{3}{2}} d_{4}^{\frac{1}{2}+\epsilon} \ll_{\epsilon} M^{3+16 \epsilon}|t|^{5+10 \epsilon} .
$$

We finally arrive at the bound

$$
\begin{aligned}
& Z_{M}\left(\frac{1}{2},-\epsilon+i t ; \chi_{a_{2} l_{2},}, \chi_{a_{1} l_{1}}\right) \\
& \quad \ll_{\epsilon} M^{3+30 \epsilon}|t|^{5+10 \epsilon} \times \\
& \quad \times \sum_{a=1,-1} \sum_{l \mid M} \sum_{\substack{d=d_{d} d_{1}^{2} \\
(d, M)=1}} \frac{\left|L\left(\frac{1}{2}, F, \chi_{d_{0}} \chi_{a l}\right) P_{d_{0}, d_{1}}^{(a l)}(1 / 2)\right|}{d^{1+\epsilon}}
\end{aligned}
$$

We now need to establish that $Z_{M}\left(\frac{1}{2}, w ; \chi_{a_{2} l_{2}}, \chi_{a_{1} l_{1}}\right)$ is analytic for $w$ in the region described in the proposition. We have already shown, in Proposition 4.10 that the product

$$
A(s, w) A(\alpha(s, w)) A(\beta(s, w)) A(\beta \alpha(s, w)) B(s, w) B\left(\alpha(s, w) \mathcal{P}(s, w) \overrightarrow{\mathbf{Z}}_{M}\left(s, w ; \chi_{\operatorname{Div}(M)}, \chi_{a_{1} l_{1}}\right) .\right.
$$

is an entire function of $s, w$. Specializing to $s=\frac{1}{2}$, we see that the only possible poles

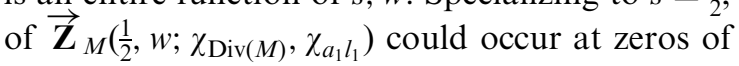

$A(1 / 2, w) A(\alpha(1 / 2, w)) A(\beta(1 / 2, w)) A(\beta \alpha(1 / 2, w)) B(1 / 2, w) B(\alpha(1 / 2, w)) \mathcal{P}(1 / 2, w)$. 
Zeros of $\mathcal{P}(1 / 2, w)$ can only occur on the real line, at $w=0, \frac{3}{4}, 1$. The other terms in the product have factors of the form $\left(1-p^{-2+2 w}\right)$ for $p \mid M$. Thus the only potential locations for poles in the region under consideration are $w=1+i t$, for a discrete sequence of $t \neq 0$. Such poles cannot occur, however, for the following reason.

For any $s, w$ with $\Re(s) \geqslant \frac{1}{2}$ and $\Re(w)>1, \overrightarrow{\mathbf{Z}}_{M}\left(s, w ; \chi_{\operatorname{Div}(M)}, \chi_{a_{1} l_{1}}\right)$ is an analytic function of $s$ and $w$. Suppose $\overrightarrow{\mathbf{Z}}_{M}\left(\frac{1}{2}, w ; \chi_{\operatorname{Div}(M)}, \chi_{a_{1} l_{1}}\right)$ has a pole of order $\gamma>0$ at $w=1+i t_{0}$. Then

$$
\lim _{(s, w) \rightarrow\left(\frac{1}{2}, l+i t_{0}\right)} \mathcal{P}_{0}(s, w) \overrightarrow{\mathbf{Z}}_{M}\left(s, w ; \chi_{\operatorname{Div}(M)}, \chi_{a_{1} l_{1}}\right) \neq 0,
$$

where $\mathcal{P}_{0}(s, w)$ is a product of $\gamma$ linear factors of the form $w-1-i t_{0}, s+w-$ $3 / 2-i t_{0}, 2 s+w-2-i t_{0}$ or $3 s+w-5 / 2-i t_{0}$. These correspond to potential zeros of the products $A(\beta(s, w)), A(\beta \alpha(s, w)), B(s, w)$ and $B(\alpha(s, w))$. By the analyticity in $s, w$, we can interchange the limits:

$$
\begin{aligned}
& \lim _{w \rightarrow l+i t_{0}} \mathcal{P}_{0}(s, w) \lim _{s \rightarrow \frac{1}{2}} \overrightarrow{\mathbf{Z}}_{M}\left(s, w ; \chi_{\operatorname{Div}(M)}, \chi_{a_{1} l_{1}}\right) \\
& \quad=\lim _{s \rightarrow \frac{1}{2}} \lim _{w \rightarrow 1+i t_{0}} \mathcal{P}_{0}(s, w) \overrightarrow{\mathbf{Z}}_{M}\left(s, w ; \chi_{\operatorname{Div}(M)}, \chi_{a_{1} l_{1}}\right) .
\end{aligned}
$$

On the right-hand side, for any $s$ with $\Re(s) \geqslant \frac{1}{2}$, let

$$
T(s)=\lim _{w \rightarrow 1+i t_{0}} \mathcal{P}_{0}(s, w) \overrightarrow{\mathbf{Z}}_{M}\left(s, w ; \chi_{\operatorname{Div}(M)}, \chi_{a_{1} l_{1}}\right) .
$$

Then $T(s)$ is an analytic function around $s=\frac{1}{2}$. Since for $\Re(s)$ sufficiently large the right-hand side of (4.10) converges absolutely, it is clear that if $\mathcal{P}_{0}(s, w)$ contains a factor of the form $w-1-i t_{0}$ then $T(s)=0$ for all such $s$. This would imply that the left-hand side equals zero, which contradicts our assumption. In a similar way we will eliminate the possibility of the other three factors dividing $\mathcal{P}_{0}(s, w)$.

By applying (4.30) to $\beta(s, w)$ and setting $w=3 / 2+i t_{0}-s$, we obtain the relation

$$
\begin{aligned}
& \prod_{p \mid M}\left(1-p^{-2\left(3 / 2+i t_{0}-s\right)}\right) \overrightarrow{\mathbf{Z}}_{M}\left(1+i t_{0}, s-1 / 2-i t_{0}\right) \\
& \quad=\Phi_{M}\left(s-1 / 2-i t_{0}\right) \overrightarrow{\mathbf{Z}}_{M}\left(s, 3 / 2+i t_{0}-s\right) .
\end{aligned}
$$

For $\Re(s)$ sufficiently large and $l_{0} \neq 0$, the left-hand side of the above converges absolutely and, hence, the right-hand side is an analytic function of $s$. Consequently, $\mathcal{P}_{0}\left(s, 3 / 2+i t_{0}-s\right)$ times the right-hand side will vanish identically if $\mathcal{P}^{0}(s, w)$ contains a factor of $s+w-3 / 2-i t_{0}$. As $\Phi\left(s-1 / 2-i t_{0}\right)$ does not vanish identically, it follows that the right-hand side of (4.36) equals zero if we approach along the line $w=3 / 2+i t_{0}-s$. This is a contradiction, so $\mathcal{P}_{0}(s, w)$ does not contain a factor of $s+w-3 / 2-i t_{0}$. 
Similarly, applying (4.29), (4.30) and setting $w=2+i t_{0}-2 s$, we obtain the relation

$$
\begin{aligned}
& \prod_{p \mid M}\left(1-p^{\left.-4+4 s-2 i t_{0}\right)}\right) \prod_{p \mid M}\left(1-p^{\left.-4+2 s-4 i t_{0}\right)}\right) \prod_{p \mid M}\left(1-p^{\left.-3+2 s-2 i t_{0}\right)}\right)^{3} \overrightarrow{\mathbf{Z}}_{M}\left(1+i t_{0}, s-1-2 i t_{0}\right) \\
& =\Phi^{\left(a_{2} l_{2}\right)}\left(2 s-1-i t_{0}\right) \Psi_{M}\left(2 s-1-i t_{0}\right) \Phi_{M}\left(2 s-1-i t_{0}\right) \overline{\mathbf{Z}}_{M}\left(s, 2+i t_{0}-2 s\right) .
\end{aligned}
$$

By the same argument as above, $\mathcal{P}_{0}(s, w)$ does not contain a factor of $2 s+w-$ $2-i t_{0}$.

Finally, applying (4.29) to $\alpha(s, w)$ and setting $w=5 / 2+i t_{0}-3 s$, we obtain the relation

$$
\prod_{p \mid M}\left(1-p^{-2 s}\right)^{3} \overrightarrow{\mathbf{Z}}_{M}\left(1-s, 1+i t_{0}\right)=\Psi_{M}(1-s) \overrightarrow{\mathbf{Z}}_{M}\left(s, 5 / 2+i t_{0}-3 s\right),
$$

from which it follows that $\mathcal{P}_{0}(s, w)$ does not contain a factor of $3 s+w-5 / 2-i t_{0}$.

The possibility of a pole at $w=0$ can be eliminated in the same way.

To see that there may, actually, be a pole at $w=\frac{3}{4}$, observe that the transformation $\alpha \beta$ relates the hyperplane $w=1$ to $3 s+2 w-3=0$. Since $w=1$ may certainly be a pole, it follows from (4.18) and (4.23) that $3 s+2 w-3=0$ may be a pole.

This establishes the analyticity of $Z\left(\frac{1}{2}, w\right)$ for $-\epsilon<\Re(w)<1+\epsilon$, except possibly at $w=\frac{3}{4}, 1$.

The upper bound follows from (4.11), (4.34) and the Phragmen-Lindelöf principle.

This completes the proof of Proposition 4.12.

\subsection{THE SIEVING PROCESS}

In this section we will use the series $Z_{M}$ as building blocks to construct

$$
Z(s, w)=\sum_{d} \frac{L\left(s, \chi d_{0}\right)^{3}}{|d|^{w}}
$$

where the sum ranges over square free integers $d_{0}$ and for each $d_{0}, d$ is the associated fundamental discriminant. This is simply the series (4.12), as $\chi_{d_{0}}=\chi_{d}$. The series $Z(s, w)$ will then inherit its analytic properties from those of $Z_{M}$.

Our object is to prove

THEOREM 4.13. Let the series $Z(s, w)$ be as defined above, and choose any $\epsilon>0$. When the specialization $s=\frac{1}{2}$ is made, $Z\left(\frac{1}{2}, w\right)$ is an analytic function of $w$ for $\Re(w)>\frac{4}{5}$ except for a pole of order 7 at $w=1$. For $w=v+i$, with $v>\frac{4}{5}, Z\left(\frac{1}{2}, w\right)$ satisfies the upper bound

$$
Z\left(\frac{1}{2}, w\right) \ll_{\epsilon} \begin{cases}1 & \text { if } 1+\epsilon<v \\ (1+|t|)^{5(1-v)+v(\epsilon)} & \text { if } \frac{4}{5}><v \leqslant 1+\epsilon\end{cases}
$$


where $v(\epsilon)$ is an explicitly computable function satisfying $\lim _{\epsilon \rightarrow 0} v(\epsilon)=0$.

Also,

$$
\lim _{w \rightarrow 1}(w-1)^{7} Z\left(\frac{1}{2}, w\right)=\frac{6 a_{3}}{4 \pi^{2}},
$$

where $a_{3}$ is given by (3.3).

In this section let $r$ denote a positive square free integer with $(r, 2)=1$. We also fix the notation $a_{1}, a_{2} \in\{1,-1\}$ and $l_{1}, l_{2} \in\{1,2\}$. Let $F$, as before, be the GL(3) Eisenstein series associated to $L\left(s, \chi d_{0}\right)^{3}$, so $L\left(s, F, \chi d_{0}\right)=L\left(s, \chi d_{0}\right)^{3}$. For any $l \mid r$, define

$$
Z_{a_{1} l_{1}, a_{2} l_{2}}^{(l)}(s, w)=\sum_{\substack{\left(d_{0}, 2\right)=1,\left(d_{1}, 2 l\right)=1 \\ d=d_{0} d_{1}^{2}}} \frac{L_{2}\left(s, F, \chi d_{0} \chi_{a_{1} l_{1}}\right) \chi_{a_{2} l_{2}}\left(d_{0}\right) P_{d_{0}, d_{1}}^{\left(a_{1} l_{1}\right)}(s)}{d^{w}}
$$

and as usual $d_{0}$ varies over positive square free integers and $d_{1}$ varies over positive integers.

If we then define

$$
Z_{a_{1} l_{1}, a_{2} l_{2}}(s, w ; r)=\sum_{l \mid r} \mu(l) Z_{a_{1} l_{1}, a_{2} l_{2}}^{(l)}(s, w),
$$

where $\mu$ denotes the usual Möbius function, it is easy to check that

$$
Z_{a_{1} l_{1}, a_{2} l_{2}}(s, w ; r)=\sum_{\substack{\left(d_{0} d_{1}, 2\right)=1, d_{1}=0(\bmod r) \\ d=d_{0} d_{1}^{2}}} \frac{L_{2}\left(s, F, \chi d_{0} \chi_{a_{1} l_{1}}\right) \chi_{a_{2} l_{2}}\left(d_{0}\right) P_{d_{0}, d_{1}}^{\left(a_{1} l_{1}\right)}(s)}{d^{w}} .
$$

In the next proposition we demonstrate that $Z_{a_{1} l_{1}, a_{2} l_{2}}^{(l)}(s, w)$, and hence $Z_{a_{1} l_{1}, a_{2} l_{2}}(s, w ; r)$ can be written as a linear combination of the functions $Z_{M}\left(s, w ; \chi_{a_{2} l_{2}}, \chi_{a_{1} l_{1}}\right)$ whose analytic properties have already been studied in the preceding sections.

PROPOSITION 4.14. We have

$$
\begin{aligned}
& Z_{a_{1} l_{1}, a_{2} l_{2}}^{(l)}(s, w) \cdot \prod_{p \mid l}\left(1-p^{-2 s}\right)^{3} \\
&= \frac{1}{2} \sum_{l_{3} \mid l} l_{3}^{-w} \prod_{p \mid l l_{3}}\left(1-p^{-2 s}\right)^{3} \times \\
& \times \sum_{m_{1}, m_{2}, m_{3} \mid\left(l / l_{3}\right)} \frac{\chi_{a_{1} l_{1} l_{3}}\left(m_{1} m_{2} m_{3}\right) \chi_{a_{2} l_{2}}\left(l_{3}\right)}{\left(m_{1} m_{2} m_{3}\right)^{s}} \times \\
& \times\left(Z_{2 l}\left(s, w ; \chi_{a_{2} l_{2}} \chi_{m_{1} m_{2} m_{3}}, \chi_{a_{1} l_{1} l_{3}}\right)+\right. \\
&+Z_{2 l}\left(s, w ; \chi_{a_{2} l_{2}} \chi_{m_{1} m_{2} m_{3}}, \chi_{a_{1} l_{1} l_{3}}\right)+ \\
&+\chi_{-1}\left(m_{1} m_{2} m_{3}\right) Z_{2 l}\left(s, w ; \chi_{a_{2} l_{2}} \chi_{m_{1} m_{2} m_{3}}, \chi_{a_{1} l_{1} l_{3}}\right)- \\
&\left.-\chi_{-1}\left(m_{1} m_{2} m_{3}\right) Z_{2 l}\left(s, w ; \chi_{a_{2} l_{2}} \chi_{-m_{1} m_{2} m_{3}}, \chi_{a_{1} l_{1} l_{3}}\right)\right) .
\end{aligned}
$$


Proof. Referring to (4.36) and (4.9), write

$$
Z_{a_{1} l_{1}, a_{2} l_{2}}^{(l)}(s, w)=\sum_{l_{3} \mid l} \sum_{\left(d_{0} d_{1}, 2 l\right)=1} \frac{L_{2}\left(s, F, \chi_{d_{0} l_{3}} \chi_{a_{1} l_{1}}\right) \chi_{a_{2} l_{2}}\left(d_{0} l_{3}\right) P_{d_{0}, d_{1}}^{\left(a_{1} l_{1} l_{3}\right)}(s)}{d_{0}^{w} l_{3}^{w} d_{1}^{2 w}} .
$$

Replacing $L_{2}\left(s, F, \chi_{d_{0} l_{3}} \chi_{a_{1} l_{1}}\right)$ by $L_{2 l}\left(s, F, \chi_{d_{0} l_{3}} \chi_{a_{1} l_{1}}\right) \cdot \prod_{p \mid l}\left(1-\chi_{d_{0} l_{3}} \chi_{a_{1} l_{1}}(p) p^{-s}\right)^{-3}$ and multiplying both sides by $\prod_{p \mid l}\left(1-p^{-2 s}\right)^{3}$, the result follows after some simple manipulations, and the use of $\chi_{-1}$ to distinguish the cases $m_{1} m_{2} m_{3} \equiv 1(\bmod 4)$ and $m_{1} m_{2} m_{3} \equiv 3(\bmod 4)$.

This completes the proof of Proposition 4.14.

It follows from Propositions 4.12, 4.14, and the definition of $Z_{a_{1} l_{1}, a_{2} l_{2}}(s, w ; r)$ in (4.37) that for $\epsilon>0$, if $w=v+i t$, with $v>-\epsilon$, then $Z_{a_{1} l_{1}, a_{2} l_{2}}(1 / 2, w ; r)$ is analytic except for possible poles at $w=\frac{3}{4}, 1$, and satisfies the upper bound

$$
Z_{a_{1} l_{1}, a_{2} l_{2}}\left(\frac{1}{2},-\epsilon+i t ; r\right) \ll_{\epsilon} r^{3+v_{3}(\epsilon)}|t|^{5+v_{4}(\epsilon)} \sum_{a=1,-1} \sum_{l \mid 2 r} \sum_{d_{0}} \frac{\left|L\left(\frac{1}{2}, \chi_{d_{0}} \chi_{a l}\right)\right|^{3}}{d_{0}^{1+\epsilon}},
$$

with $v_{3}(\epsilon), v_{4}(\epsilon)$ some explicitly computable functions satisfying $\lim _{\epsilon \rightarrow 0} v_{3}(\epsilon)=$ $\lim _{\epsilon \rightarrow 0} v_{4}(\epsilon)=0$. For $v>1$, the series $Z_{a_{1} l_{1}, a_{2} l_{2}}(1 / 2, w ; r)$ converges absolutely, by (4.11) and (4.33), and a factor of $r^{2 v}$ factors out of the denominator. Thus $Z_{a_{1} l_{1}, a_{2} l_{2}}(1 / 2,1+\epsilon+i t ; r) \ll_{\epsilon} r^{-2-2 \epsilon}$. Combining these bounds and applying Phragmen-Lindelöf, we obtain, for $-\epsilon<v<1+\epsilon$ and $|t|>1$,

$$
Z_{a_{1} l_{1}, a_{2} l_{2}}\left(\frac{1}{2}, v+i t ; r\right) \ll_{\epsilon} r^{3-5 v+v_{3}(\epsilon)}|t|^{5-5 v+v_{4}(\epsilon)} \sum_{a=1,-1} \sum_{l \mid 2 r} \sum_{d_{0}} \frac{\left|L\left(\frac{1}{2}, \chi_{d_{0}} \chi_{a l}\right)\right|^{3}}{d_{0}^{1+\epsilon}} .
$$

We now define

$$
Z_{a_{1} l_{1}, a_{2} l_{2}}(s, w)=\sum_{(r, 2)=1} \mu(r) Z_{a_{1} l_{1}, a_{2} l_{2}}(s, w ; r),
$$

and observe that

$$
Z_{a_{1} l_{1}, a_{2} l_{2}}(s, w)=\sum_{\left(d_{0}, 2\right)=1} \frac{L_{2}\left(s, \chi_{d_{0}} \chi_{a_{1} l_{1}}\right)^{3} \chi_{a_{2} l_{2}}\left(d_{0}\right)}{d_{0}^{w}},
$$

where the sum is over odd, square free positive integers $d_{0}$. The sum over $r$ has removed all $d_{1} \neq 1$ from the sum. Applying the bound of (4.39) and taking $v>v_{0}>\frac{4}{5}$, we have

$$
\begin{aligned}
Z_{a_{1} l_{1}, a_{2} l_{2}}\left(\frac{1}{2}, v+i t\right) & \ll_{\epsilon}|t|^{5-5 v+v_{4}(\epsilon)} \sum_{(r, 2)=1}(2 r)^{3-5 v+v_{3}(\epsilon)} \sum_{a=1,-1} \sum_{l \mid 2 r} \sum_{d_{0}} \frac{\left|L\left(\frac{1}{2}, \chi_{d_{0}} \chi_{a l}\right)\right|^{3}}{d_{0}^{1+\epsilon}} \\
& \ll_{\epsilon}|t|^{5-5 v+v_{4}(\epsilon)} \sum_{a=1,-1} \sum_{l} \sum_{d_{0}} \frac{\left|L\left(\frac{1}{2}, \chi_{d_{0}} \chi_{a l}\right)\right|^{3}}{d_{0}^{1+\epsilon} l^{5 v-3-v_{3}(\epsilon)}} \sum_{r^{\prime} \geqslant 1} \frac{1}{r^{\prime 5 v-3-v_{3}(\epsilon)}} \\
& \ll_{v_{0}, \epsilon}|t|^{5-5 v+v_{4}(\epsilon)},
\end{aligned}
$$


if $\epsilon$ is chosen sufficiently small. In (4.40), the last estimate follows from (4.33).

We have thus proved

PROPOSITION 4.15. For any $a_{1}, a_{2} \in\{1,-1\}$ and $l_{1}, l_{2} \in\{1,2\}$, the series $Z_{a_{1} l_{1}, a_{2} l_{2}}\left(\frac{1}{2}, w\right)$ is analytic for $w=v+$ it when $v>\frac{4}{5}$, except possibly for a pole at $w=1$. For $|t|>1$ it satisfies the upper bound

$$
Z_{a_{1} l_{1}, a_{2} l_{2}}\left(\frac{1}{2}, v+i t\right) \ll_{\epsilon}|t|^{5-5 v+v_{4}(\epsilon)} .
$$

To complete the proof of the first part of Theorem 4.13, we make choices of $1,-1,2,-2$ for $a_{1} l_{1}$ and $a_{2} l_{2}$ and take linear combinations of $Z_{a_{1} l_{1}, a_{2} l_{2}}(1 / 2, w)$ to isolate sums over $d_{0}>0, d_{0}<0$, and for each sign, sums over $d_{0} \equiv 1(\bmod 8), d_{0} \equiv 5$ $(\bmod 8), d_{0} \equiv 3(\bmod 4)$ and $d_{0} \equiv 1(\bmod 4)$. After these sums are isolated, the 2-factor of the $L$-series can be restored, and the analyticity of $Z\left(\frac{1}{2}, w\right)$ for $w \neq 1$ together with the upper bound stated in Theorem 4.13 follows.

It now remains to calculate the order of the pole and compute the leading coefficient in the Laurent expansion at $w=1$. This can be done directly from the analytic information and functional equations we have accumulated about $Z_{a_{1} l_{1}, a_{2} l_{2}}(s, w)$. However, it is an intricate computation, and so we will instead make use of the computations already performed in Section 3 for a general multiple Dirichlet series.

In the notation of Section 3, taking $m=3, Z(s, w)=Z(s, s, s, w)$, where $Z\left(s_{1}, s_{2}, s_{3}, w\right)$ is defined by (3.6). In the previous work of this section we considered the $L$-series $L(s, F)=\zeta(s)^{3}$. Here $F$ was an Eisenstien series on GL(3) specialized to the center of the critical strip. We could just have easily have considered the $L$-series associated to $F^{\prime}$, a general minimal parabolic Eisenstein series. In the case of $F$, the Euler product parameters at a prime $p$ were $\alpha_{p}=\beta_{p}=\gamma_{p}=1$ and the corresponding local factor of the Euler product was $\left(1-p^{-s}\right)^{-3}$. For the more general $F^{\prime}$, we can take $\alpha_{p}=p^{-\epsilon_{1}}, \beta_{p}=p^{-\epsilon_{2}}, \gamma_{p}=p^{\epsilon_{1}+\epsilon_{2}}$. The corresponding local factor of $L\left(s, F^{\prime}\right)$ is then equal to $\left(\left(1-p^{-s-\epsilon_{1}}\right)\left(1-p^{-s-\epsilon_{2}}\right)\left(1-p^{-s+\epsilon_{1}+\epsilon_{2}}\right)\right)^{-1}$. Applying exactly the same arguments as before, we may obtain the analytic continuation of the more general object

$$
Z\left(s+\epsilon_{1}, s+\epsilon_{2}, s-\epsilon_{1}-\epsilon_{2}, w\right)=\sum_{d} \frac{L\left(s+\epsilon_{1}, \chi_{d_{0}}\right) L\left(s+\epsilon_{2}, \chi_{d_{0}}\right) L\left(s-\epsilon_{1}-\epsilon_{2}, \chi_{d_{0}}\right)}{|d|^{w}}
$$

in a neighbourhood of $s=1 / 2$ and $\epsilon_{1}=\epsilon_{2}=0$. Setting $s_{1}=s+\epsilon_{1}, s_{2}=s+\epsilon_{2}$ and $s_{3}=s-\epsilon_{1}-\epsilon_{2}$, we are in a position to take advantage of the calculations done in Section 3, as we have established the conjectured analytic continuation. This completes the proof of Theorem 4.13.

It is worth remarking that we could just as easily have proved the more general analytic continuation of $Z\left(s_{1}, s_{2}, s_{3}, w\right)$. However, our intent was to make the outlines of the technique as clear as possible. Writing out the explicit details in greater generality would have made it significantly harder to distinguish the ideas through the notation. 
We now have only a small additional piece of work to do to complete the proof of the first part of Theorem 1.1. Applying the integral transform

$$
\frac{1}{2 \pi i} \int_{2-i \infty}^{2+i \infty} \frac{x^{w} \mathrm{~d} w}{w(w+1)}= \begin{cases}(1-1 / x) & \text { if } \quad x>1 \\ 0 & \text { if } \quad 0<x \leqslant 1\end{cases}
$$

we obtain first

$$
\frac{1}{2 \pi i} \int_{2-i \infty}^{2+i \infty} \frac{Z(1 / 2, w) x^{w} \mathrm{~d} w}{w(w+1)}=\sum_{|d|<x} L\left(\frac{1}{2}, \chi_{d}\right)^{3}\left(1-\frac{|d|}{x}\right) .
$$

Moving the line of integration to $\Re(w)=\frac{4}{5}+\epsilon$, for $\epsilon>0$, we pick up from the pole at $w=1$ a polynomial type expression of the form $x\left(A_{6}(\log x)^{6}+A_{5}(\log x)^{5}\right.$ $\left.+\cdots+A_{0}\right)$, where the constants $A_{6}, \ldots, A_{0}$ are computable and

$$
A_{6}=\frac{6 a_{3}}{8 \pi^{2} 6 !}
$$

i.e., $1 / 2$ the constant of Theorem 4.13 , divided by 6 !. The integral at $\Re(w)=\frac{4}{5}+\epsilon$ converges absolutely by the upper bound estimate of Theorem 4.13, and contributes an error on the order of $x^{\frac{4}{5}+\epsilon}$. This completes the proof of the first part of Theorem 1.1

\subsection{AN UNWEIGHTED ESTIMATE}

In this section we will prove the second part of Theorem 1.1. An essential ingredient of an estimate for such a theorem, and, more generally, an estimate for an unweighted sum $\sum_{d<x} a_{d}$ when $a_{d}$ is not known to be non-negative, is an estimate for sums of $a_{d}$ over short intervals. In our case, if $d$ is square free then $a_{d}=L\left(1 / 2, \chi_{d}\right)^{3}$, while if $d=d_{0} d_{1}^{2}$ with $d_{0}$ square free, then

$$
a_{d}=L\left(1 / 2, \chi_{d_{0}}\right)^{3} P_{d_{0}, d_{1}}(1 / 2),
$$

where $d^{-\epsilon} \ll P_{d_{0}, d_{1}}(1 / 2) \ll d^{\epsilon}$. Here $P_{d_{0}, d_{1}}(1 / 2)$ is a linear combination of $P_{d_{0}, d_{1}}^{\left(a_{1} l_{1}\right)}(1 / 2)$. As a first step we will require the following.

PROPOSITION 4.16. For $x>0$ sufficiently large, $\epsilon>0$, and $\frac{3}{5}<\theta_{0} \leqslant 1$,

$$
\sum_{|d-x|<x^{\theta_{0}}} L\left(1 / 2, \chi_{d_{0}}\right)^{2} \ll_{\epsilon} x^{\theta_{0}+\epsilon}
$$

The sum here is over $d$ of the form $d=d_{0} m^{2}$ for some $m$, with $d_{0}$ square free and either positive or negative.

Proof. The easiest way to prove the Proposition is to apply Theorem 4.1 of [C-N] to the analog of $Z_{M}\left(s, w ; \chi_{1}, \chi_{1}\right)$ of (4.13) in the case of GL(2), i.e., when $L_{M}\left(s, F, \chi_{d_{0}}\right)=L_{M}\left(s, \chi_{d_{0}}\right)^{2}$ for $d_{0}$ square free. Then all coefficients are nonnegative. 
There are four gamma factors, so $A=2$ in their notation, and the result with exponent $3 / 5$ follows immediately, by ignoring all but the square free terms. (The sum over $m$ does not affect the exponent.) The derivation of the analytic continuation and functional equation of $Z_{M}\left(s, w ; \chi_{1}, \chi_{1}\right)$ is done precisely as in the preceding sections and is omitted. Alternatively, and more traditionally, one could obtain this analytic continuation by considering the Rankin-Selberg convolution of a half-integral weight Eisenstein series with itself. The analysis, however, is considerably more complicated.

Fix an $x$, and $r<\sqrt{x}$. The following Proposition will begin the proof of our estimate for unweighted sums of coefficients of $Z_{a_{1} l_{1}, a_{2} l_{2}}(s, w ; r)$. To simplify notation we will suppress $a_{1}, a_{2}, l_{1}, l_{2}$ and write

$$
a(d)=L_{2}\left(1 / 2, \chi_{d_{0}} \chi_{a_{1} l_{1}}\right)^{3} \chi_{a_{2} l_{2}}\left(d_{0}\right) P_{d_{0}, d_{1}}^{\left(a_{1} l_{1}\right)}(1 / 2) .
$$

Thus

$$
Z_{a_{1} l_{1}, a_{2} l_{2}}(1 / 2, w ; r)=\sum_{\substack{\left(d_{0} d_{1}, 2\right)=1, d_{1}=0(\bmod r) \\ d=d_{0} d_{1}^{2}}} \frac{a(d)}{d^{w}} .
$$

PROPOSITION 4.17. Fix $x, T>0, r$ square free, $a_{1}, a_{2} \in\{1,-1\}, l_{1}, l_{2} \in\{1,2\}$, and $\epsilon>0$. Let

$$
I_{1}(r)=\frac{1}{2 \pi i} \int_{1+\epsilon-i T}^{1+\epsilon+i T} \frac{Z_{a_{1} l_{1}, a_{2} l_{2}}(1 / 2, w ; r) x^{w} \mathrm{~d} w}{w} .
$$

Then for any $1 \geqslant \theta_{0}>3 / 5$

$$
I_{1}(r)=\sum_{d<x, d=0\left(\bmod r^{2}\right)} a(d)+\mathcal{O}_{\epsilon}\left(x^{\epsilon} r^{\epsilon}\left(\frac{x}{r^{2}}\right)^{\left(1+\theta_{0}\right) / 2}\right)+\mathcal{O}_{\epsilon}\left(x^{\epsilon} r^{\epsilon} \frac{1}{T}\left(\frac{x}{r^{2}}\right)^{\left(3-\theta_{0}\right) / 2}\right) .
$$

Proof. Applying the integral transform

$$
\frac{1}{2 \pi i} \int_{1+\epsilon-i T}^{1+\epsilon+i T} \frac{x^{w} \mathrm{~d} w}{w}=\left\{\begin{array}{lll}
1 & \text { if } & x>1, \\
0 & \text { if } & 0<x<1
\end{array}+\mathcal{O}_{\epsilon}\left(x^{1+\epsilon} \min \left(1, \frac{1}{T|\log (x)|}\right)\right)\right.
$$

to $Z_{a_{1} l_{1}, a_{2} l_{2}}(1 / 2, w ; r)$ and interchanging the order of summation and integration, as we are in a region of absolute convergence, we obtain

$$
I_{1}(r)=\sum_{d<x, d \equiv 0\left(\bmod r^{2}\right)} a(d)+E_{1},
$$

where

$$
E_{1} \ll_{\epsilon} \sum_{d \equiv 0\left(\bmod r^{2}\right), d \neq 0}|a(d)|\left(\frac{x}{d}\right)^{1+\epsilon} \min \left(1, \frac{1}{T|\log (x / d)|}\right) .
$$


Break the sum $E_{1}$ into three pieces: $E_{1}=E_{2}+E_{3}+E_{4}$, where the sums are over $d<\frac{1}{2} x, d>2 x$ and $\frac{1}{2} x<d<2 x$, respectively. Write $d=d_{0} m^{2} r^{2}$, with $d_{0}$ square free. By its definition in (4.42), together with the bound of (4.11), we have the bound

$$
a(d) \ll_{\epsilon}\left|L\left(1 / 2, \chi_{d_{0}} \chi_{a_{1} l_{1}}\right)\right|^{3} \cdot d^{\epsilon} .
$$

Applying (4.44) to $E_{2}, E_{3}$, we see that $E_{2}, E_{3} \ll_{\epsilon} x^{1+\epsilon} r^{-2-2 \epsilon} T^{-1}$ follows immediately from the absolute convergence of $\sum L\left(1 / 2, \chi_{d_{0}}\right)^{3}\left|d_{0}\right|^{-1-\epsilon}$ (which follows, as remarked before, from Heath-Brown's results [H-B]).

To analyze $E_{4}$, note that we are summing over the range $\frac{1}{2} x r^{-2}<d_{0} m^{2}<2 x r^{-2}$, so

$$
E_{4} \ll_{\epsilon} \sum_{d \equiv 0\left(\bmod r^{2}\right), \frac{1}{2} x<d<2 x}|a(d)| \cdot \min \left(1, \frac{1}{T|\log (x / d)|}\right) .
$$

We are summing over the range $\frac{1}{2} x r^{-2}<\mathrm{d} r^{-2}=d_{0} m^{2}<2 x r^{-2}$. Consequently, for any $\theta_{0}>0$ we may write $d_{0} m^{2}=\left[x r^{-2}+d^{\prime}\left(x r^{-2}\right)^{\theta_{0}}+d^{\prime \prime}\right]$. As $d^{\prime}, d^{\prime \prime}$ vary over the ranges $0 \leqslant\left|d^{\prime}\right| \ll\left(x r^{-2}\right)^{1-\theta_{0}}$ and $0 \leqslant d^{\prime \prime} \ll\left(x r^{-2}\right)^{\theta_{0}}$, the full range of values of $d_{0} m^{2}$ will be hit. We will treat the cases $d^{\prime}=0,-1$ and $d^{\prime} \neq 0,-1$ separately.

Write $E_{4}=E_{5}+E_{6}$ where $E_{5}$ is the sum over $d$ with $d^{\prime}=0,-1$. The choosing 1 in the minimum of (4.45) we have

$$
E_{5} \ll \sum_{d^{\prime}=0,-1} \sum_{0 \leqslant d^{\prime \prime} \ll\left(x r^{-2}\right)^{\theta_{0}}}|a(d)|=\sum^{*}|a(d)|,
$$

where $\sum^{*}$ denotes the sum ranging over $d^{\prime}, d_{0}, m$ satisfying $d^{\prime}=0,-1$ and

$$
0 \leqslant\left|d_{0} m^{2}-x r^{-2}-d^{\prime}\left(x r^{-2}\right)^{\theta_{0}}\right| \ll\left(x r^{-2}\right)^{\theta_{0}} .
$$

Also, by (4.44)

$$
a(d) \ll_{\epsilon} r^{\epsilon} x^{\epsilon}\left|L\left(1 / 2, \chi_{d_{0}} \chi_{a_{1} l_{1}}\right)\right|^{3} .
$$

It follows by the Cauchy-Schwartz inequality that

$$
E_{5} \ll_{\epsilon} r^{\epsilon} x^{\epsilon}\left(\sum^{* *}\left|L\left(1 / 2, \chi_{d_{0}} \chi_{a_{1} l_{1}}\right)\right|^{4}\right)^{1 / 2}\left(\sum^{* *}\left|L\left(1 / 2, \chi_{d_{0}} \chi_{a_{1} l_{1}}\right)\right|^{2}\right)^{1 / 2},
$$

where $\sum^{* *}$ denotes the sum ranging over $d_{0}, m$ satisfying the condition

$$
\left|d_{0}-\frac{x r^{-2}}{m^{2}}\right| \ll \frac{\left(x r^{-2}\right)^{\theta_{0}}}{m^{2}} .
$$

Using [H-B] to bound the sum of fourth powers by $x$, and using Proposition 4.16 to bound the sum over squares we obtain

$$
E_{5} \ll_{\epsilon} r^{\epsilon} x^{\epsilon}\left(\frac{x}{r^{2}}\right)^{\left(1+\theta_{0}\right) / 2} \sum_{m=1}^{\infty} m^{-1-\theta_{0}} \ll_{\epsilon} r^{\epsilon} x^{\epsilon}\left(\frac{x}{r^{2}}\right)^{\left(1+\theta_{0}\right) / 2} .
$$

To bound $E_{6}$ we first use the same argument as above to bound the sum over $d^{\prime \prime}$ for fixed $d^{\prime}$. We then observe that for $d^{\prime} \neq 0,-1$ and any $d^{\prime \prime}$ we have $|\log (d / x)|^{-1} \ll$ 
$\left(x r^{-2}\right)^{1-\theta_{0}} /\left|d^{\prime}\right|$. Taking the log term in the minimum of (4.45) and summing over $d^{\prime} \neq 0$ we obtain

$$
E_{6} \ll_{\epsilon} r^{\epsilon} x^{\epsilon}\left(\frac{x}{r^{2}}\right)^{\left(1+\theta_{0}\right) / 2} T^{-1} \sum_{d^{\prime} \neq 0,-1}\left(x r^{-2}\right)^{1-\theta_{0}} /\left|d^{\prime}\right| \ll_{\epsilon} r^{\epsilon} x^{\epsilon} T^{-1}\left(\frac{x}{r^{2}}\right)^{\left(3+\theta_{0}\right) / 2} .
$$

This completes the proof of Proposition 4.17.

Continuing with the proof of the Theorem, we now define, for $\epsilon>0$, and any $-\epsilon \leqslant \sigma \leqslant 1-\epsilon$

$$
I_{2}(r, \sigma)=\frac{1}{2 \pi i} \int_{\sigma-i T}^{\sigma+i T} \frac{Z_{a_{1} l_{1}, a_{2} l_{2}}(1 / 2, w ; r) x^{w} \mathrm{~d} w}{w}
$$

and

$$
\begin{aligned}
& I_{3}(r, \sigma)=\frac{1}{2 \pi i} \int_{\sigma+i T}^{1+\epsilon+i T} \frac{Z_{a_{1} l_{1}, a_{2} l_{2}}(1 / 2, w ; r) x^{w} \mathrm{~d} w}{w}, \\
& I_{4}(r, \sigma)=\frac{1}{2 \pi i} \int_{1+\epsilon-i T}^{\sigma-i T} \frac{Z_{a_{1} l_{1}, a_{2} l_{2}}(1 / 2, w ; r) x^{w} \mathrm{~d} w}{w} .
\end{aligned}
$$

Thus,

$$
\begin{aligned}
I_{1}(r)= & x \sum_{i=0}^{6} d_{i}(r)(\log x)^{i}+I_{2}(r, \sigma)+I_{3}(r, \sigma)+ \\
& +I_{4}(r, \sigma)+\delta_{\sigma} \cdot \frac{4}{3} x^{\frac{3}{4}} \cdot \underset{w=\frac{3}{4}}{\operatorname{Res}\left(Z_{a_{1} l_{1}, a_{2} l_{2}}(1 / 2, w ; r)\right),}
\end{aligned}
$$

for some computable constants $d_{i}(r)$. The main term is contributed by the seventh order pole at $w=1$ and the residue term comes from the possible pole at $w=\frac{3}{4}$, provided $-\epsilon<\sigma<\frac{3}{4}-\epsilon$ for some sufficiently small $\epsilon>0$. Here $\delta_{\sigma}=1$ if $-\epsilon<\sigma<\frac{3}{4}-\epsilon$ and $\delta_{\sigma}=0$, otherwise. Note that there is no pole at $w=0$, so there are no additional error terms.

It immediately follows from Proposition 4.17 and (4.49) that

$$
\begin{aligned}
\sum_{\substack{d<x \\
d \text { square free }}} a_{d}= & \sum_{r \leqslant \sqrt{x}} \mu(r)\left[x \sum_{i=0}^{6} d_{i}(r)(\log x)^{i}+I_{2}(r, \sigma)+I_{3}(r, \sigma)+I_{4}(r, \sigma)+\right. \\
& +\delta_{\sigma} \cdot \frac{4}{3} x^{\frac{3}{4}} \cdot \underset{\operatorname{Res}_{w=\frac{3}{4}}}{\operatorname{Res}\left(Z_{a_{1} l_{1}, a_{2} l_{2}}(1 / 2, w ; r)\right)+\mathcal{O}_{\epsilon}\left(x^{\epsilon} r^{\epsilon}\left(\frac{x}{r^{2}}\right)^{\left(1+\theta_{0}\right) / 2}\right)+} \\
& \left.+\mathcal{O}_{\epsilon}\left(x^{\epsilon} r^{\epsilon} \frac{1}{T}\left(\frac{x}{r^{2}}\right)^{\left(3-\theta_{0}\right) / 2}\right)\right]
\end{aligned}
$$

The sum $\sum_{r \leqslant \sqrt{x}} \mu(r) x \sum_{i=0}^{6} d_{i}(r)(\log x)^{i}$ will give the main term of the second part of Theorem 1.1 with a negligible error of $O\left(x^{\frac{1}{2}+\epsilon}\right)$. Thus, to complete the proof of 
Theorem 1.1 it remains to estimate the integrals and error terms in (4.50). These will be estimated by breaking the sum over $r$ into $1 \leqslant r \leqslant x^{\gamma}$ and $x^{\gamma}<r \leqslant \sqrt{x}$ for some $0<\gamma \leqslant \frac{1}{2}$ to be chosen later. We note that we will make different choices of $T$ and $\sigma$ depending on whether $1 \leqslant r \leqslant x^{\gamma}$ or $x^{\gamma}<r \leqslant \sqrt{x}$. After computing all the error terms, we will make an optimal choice of the variables $\gamma, \sigma, T, \theta_{0}$.

In order to estimate the integrals in (4.50), we make use of the upper bound (4.39). It follows that for $-\epsilon \leqslant v<1$,

$$
\begin{aligned}
& Z_{a_{1} l_{1}, a_{2} l_{2}}\left(\frac{1}{2}, v+i t ; r\right) \\
& \quad \ll_{\epsilon} r^{3-5 v+v_{3}(\epsilon)}(1+|t|)^{5-5 v+v_{4}(\epsilon)} \sum_{a=1,-1} \sum_{l \mid 2 r} \sum_{d_{0}} \frac{\left|L\left(\frac{1}{2}, \chi_{d_{0}} \chi_{a l}\right)\right|^{3}}{d_{0}^{1+\epsilon}} .
\end{aligned}
$$

PROPOSITION 4.18. Let $x, T>0, r$ square free, and $\epsilon>0$. The integral $I_{2}(r,-\epsilon)$ given in (4.48) satisfies

$$
\begin{aligned}
I_{2}(r,-\epsilon)= & \frac{1}{2 \pi i} \int_{-\epsilon-i T}^{-\epsilon+i T} \frac{Z_{a_{1} l_{1}, a_{2} l_{2}}(1 / 2, w ; r) x^{w} \mathrm{~d} w}{w} \\
& \ll_{\epsilon} r^{3+v_{5}(\epsilon)} T^{\frac{9}{2}+v_{6}(\epsilon)} \sum_{a=1,-1} \sum_{l \mid 2 r} \sum_{d_{0}} \frac{\left|L\left(\frac{1}{2}, \chi_{d_{0}} \chi_{a l}\right)\right|^{3}}{d_{0}^{1+\epsilon}},
\end{aligned}
$$

where $v_{5}(\epsilon)$ and $v_{6}(\epsilon)$ are some explicitly computable functions satisfying

$$
\lim _{\epsilon \rightarrow 0} v_{5}(\epsilon)=\lim _{\epsilon \rightarrow 0} v_{6}(\epsilon)=0 .
$$

Proof. The ultimate effect of this proposition is to save a power of $T^{1 / 2}$ in the estimate for $I_{2}$. To accomplish this, our goal is to apply the functional equation (4.31) to $Z_{a_{1} l_{1}, a_{2} l_{2}}(1 / 2,-\epsilon+i t ; r)$, reflecting it into a region where it converges absolutely. This functional equation reflects $Z$ into a new series which is actually a linear combination of convergent series. This combination is summed over divisors of $2 r$ and also over ratios of gamma factors corresponding to $L$ series with both positive and negative conductors. The easiest way to deal with this is to use the following notation:

Let $\vec{\beta}=\left(\beta_{1}, \beta_{2}, \beta_{3}, \beta_{4}, \beta_{5}\right)$, where each $\beta_{i} \in\{0,1\}$. Let $\Delta_{\vec{\beta}}$ denote the product of gamma factors

$$
\Delta_{\vec{\beta}}(w)=G\left(w+\beta_{1}\right) G\left(w+\beta_{2}\right)^{3} G\left(2 w-1 / 2+\beta_{3}\right) G\left(w+\beta_{4}\right)^{3} G\left(w+\beta_{5}\right),
$$

where $G(w)=\pi^{-w / 2} \Gamma(w / 2)$.

Then for fixed $x$ and $T$, it follows from (4.31) and the explicit forms of the functional equations of Proposition 4.2 and 4.3 given by (4.18) and (4.23) that we may reflect $Z_{a_{1} l_{1}, a_{2} l_{2}}(1 / 2, w ; r)$ into a complicated sum of Dirichlet series evaluated at $1-w$. 
By a similar argument to the one given in the proof of Proposition 4.13, it can be observed that the bound for the integral $I_{2}$ follows, if we show the estimate

$$
I_{\vec{\beta}}(y, T, \epsilon):=\int_{T}^{T} \frac{\Delta_{\vec{\beta}}(1+\epsilon+i t)}{\Delta_{\vec{\beta}}(-\epsilon-i t)} \cdot \frac{y^{i t}}{\epsilon+i t} \mathrm{~d} t \ll_{\epsilon} T^{\frac{9}{2}+10 \epsilon},
$$

where $y$ is any positive number.

To prove the estimate (4.52), we first observe that from Stirling's formula, we have

$$
\frac{\Delta_{\vec{\beta}}(1+\epsilon+i t)}{\Delta_{\vec{\beta}}(-\epsilon-i t)}=|t|^{5+10 \epsilon+10 i t} \mathrm{e}^{c i t} c^{\prime}(\epsilon, \vec{\beta})\left\{1+\mathcal{O}\left(\frac{1}{|t|}\right)\right\},
$$

for certain constants, $c, c^{\prime}(\epsilon, \vec{\beta})$.

Replacing the ratio on the left-hand side of (4.53) with the main term, the contribution from the error term is easily seen to be bounded above by $\mathcal{O}\left(T^{4+\epsilon}\right)$, and using the expansion

$$
\frac{1}{\epsilon+i t}=-\frac{i}{t}\left(1+\left(\frac{i \epsilon}{t}\right)+\left(\frac{i \epsilon}{t}\right)^{2}+\cdots\right)
$$

it is enough to prove that

$$
\int_{1}^{T} t^{u+10 i t} y^{i t} \mathrm{~d} t \ll\left\{\begin{array}{lll}
T^{u+\frac{1}{2}} & \text { if } \quad u \geqslant 0, \\
T^{\frac{1}{2}} & \text { if } \quad u<0 .
\end{array}\right.
$$

This is a simple consequence of the following lemma $[\mathrm{T}]$.

LEMMA 4.19. Let $F(x)$ be a real function, twice differentiable, and let $F^{\prime \prime}(x) \geqslant$ $m>0$, or $F^{\prime \prime}(x) \leqslant-m<0$, for any $x, a \leqslant x \leqslant b$. Let $G(x) / F^{\prime}(x)$ be monotonic, and $|G(x)| \leqslant M$. Then

$$
\left|\int_{a}^{b} G(x) \mathrm{e}^{i F(x)} \mathrm{d} x\right| \leqslant \frac{8 M}{\sqrt{m}} .
$$

Choosing $F(t)=t(10 \log t+\log y)$ and $G(t)=t^{u}$, we can divide the interval [1,T] in several subintervals such that the conditions in the Lemma 4.19 are satisfied in each subinterval. The bound (4.54) follows:

This completes the proof of Proposition 4.18.

LEMMA 4.20. Let $0<\gamma<\rho$ and $x \rightarrow \infty$. Then for any $\epsilon>0$,

$$
\sum_{x^{\gamma} \leqslant r \leqslant x^{\rho}} r^{u} \sum_{a=1,-1} \sum_{l \mid 2 r} \sum_{d_{0}} \frac{\left|L\left(\frac{1}{2}, \chi_{d_{0}} \chi_{a l}\right)\right|^{3}}{d_{0}^{1+\epsilon}} \ll_{\epsilon}\left\{\begin{array}{lll}
x^{\rho(u+1)+\epsilon} & \text { if } \quad u>-1, \\
x^{\gamma(u+1)+\epsilon} & \text { if } \quad u<-1 .
\end{array}\right.
$$


Proof. Let $S$ denote the quadruple sum given above. By interchanging sums and writing $2 r=l \cdot r_{1}$, we easily see that

$$
\begin{aligned}
S & =\sum_{a=1,-1} \sum_{l \leqslant 2 x^{\rho}} \sum_{d_{0}} 2^{-u} \sum_{2 r=0(l)}(2 r)^{u} \cdot \frac{\left|L\left(\frac{1}{2}, \chi_{d_{0}} \chi_{a l}\right)\right|^{3}}{d_{0}^{1+\epsilon}} \\
& \ll \sum_{a=1,-1} \sum_{l \leqslant 2 x^{\rho}} \sum_{d_{0}} \sum_{\frac{2}{T} x^{v} \leqslant r_{1} \leqslant \frac{2}{T} x^{\rho}} l^{u+1+\epsilon} r_{1}^{u} \cdot \frac{\left|L\left(\frac{1}{2}, \chi_{d_{0}} \chi_{a l}\right)\right|^{3}}{d_{0}^{1+\epsilon}} .
\end{aligned}
$$

Now, if $u<-1$, the inner sum over $r_{1}$ is a convergent series which is bounded by $x^{\gamma(u+1)+\epsilon}$. The remaining sums are absolutely convergent and bounded by (4.33). This establishes the first case of the Lemma.

If $u>-1$, then the inner sum over $r_{1}$ is bounded by $\left(\frac{2}{l} x^{\rho}\right)^{u+1+\epsilon}$. The result then again immediately follows from (4.33). This completes the proof of Lemma 4.20.

We now proceed to systematically estimate the integrals and error terms in (4.50). Consider first the case $r>x^{\gamma}$ for some $\gamma$ to be determined later. Choosing $T=$ $x^{\left(3-\theta_{0}\right) / 2}, \sigma=1-\epsilon$, and summing over $x^{\gamma}<\gamma \leqslant x^{\frac{1}{2}}$, we find that the error contributions.

$$
\mathcal{O}_{\epsilon}\left(x^{\epsilon} r^{\epsilon}\left(\frac{x}{r^{2}}\right)^{\frac{1+\theta_{0}}{2}}\right), \quad \mathcal{O}_{\epsilon}\left(x^{\epsilon} r^{\epsilon} \frac{1}{T}\left(\frac{x}{r^{2}}\right)^{\frac{3-\theta_{0}}{2}}\right)
$$

are dominated by the first, which contributes (changing $\epsilon$ as appropriate)

$$
\sum_{x^{\gamma} \leqslant r \leqslant x^{\frac{1}{2}}} x^{\epsilon} r^{\epsilon}\left(\frac{x}{r^{2}}\right)^{\frac{1+\theta_{0}}{2}} \ll_{\epsilon} x^{\frac{1+\theta_{0}}{2}-\gamma \theta_{0}+\epsilon} .
$$

Applying 4.51 and Lemma 4.20 to the definition of $I_{2}(r, \sigma)$ given in (4.48), it follows that

$$
\sum_{x^{\prime} \leqslant r \leqslant x^{\frac{1}{2}}}\left|I_{2}(r, 1-\epsilon)\right| \ll_{\epsilon} x^{1-\gamma+\epsilon},
$$

again changing $\epsilon$ as appropriate. Similarly, using (4.51) and Lemma 4.20, the integrals $I_{3}(r, 1-\epsilon)$ and $I_{4}(r, 1-\epsilon)$ contribute a smaller amount than the above error terms.

Finally, we consider the case when $r<x^{\gamma}$. For this case, we choose $\sigma=-\epsilon, T=\frac{x^{\alpha}}{r^{\beta}}$ with $\alpha-\beta \gamma>0$ where $0<\alpha, \beta$ will be chosen later. First, we consider the error from the pole at $w=\frac{3}{4}$. It follows from (4.51) and Lemma 4.20 that the contribution is bounded by

$$
\sum_{r<x^{\eta}} r^{-\frac{3}{4}+\epsilon} x^{\frac{3}{4}} \ll x^{\frac{\gamma}{4}+\frac{3}{4}+\epsilon}
$$

This error will be negligible compare to the others and can be discarded. The error coming from the $I_{2}$ integral can be estimated using Proposition 4.18 and Lemma 4.20. We obtain 


$$
\begin{aligned}
\sum_{r<x^{\eta}} I_{2}(r,-\epsilon) & \ll x^{\frac{9}{2} \alpha+\epsilon} \sum_{r<x^{\gamma}} r^{3-\frac{9}{2} \beta+\epsilon} \sum_{a=1,-1} \sum_{l \mid 2 r} \sum_{d_{0}} \frac{\left|L\left(\frac{1}{2}, \chi_{d_{0}} \chi_{a l}\right)\right|^{3}}{d_{0}^{1+\epsilon}} \\
& \ll x^{\frac{9}{2} \alpha} \begin{cases}x^{\gamma\left(4-\frac{9}{2} \beta+\epsilon\right)} & \text { if } \beta<\frac{8}{9} \\
x^{\epsilon} & \text { if } \quad \beta<\frac{8}{9} .\end{cases}
\end{aligned}
$$

We now estimate the errors contributed by (4.55). First

$$
\sum_{r<x^{\vartheta}} x^{\epsilon} r^{\epsilon}\left(\frac{x}{r^{2}}\right)^{\frac{1+\theta_{0}}{2}} \ll x^{\frac{1+\theta_{0}}{2}+\epsilon}
$$

Secondly, we have

$$
\begin{aligned}
\sum_{r<x^{\gamma}} x^{\epsilon} r^{\epsilon} \frac{1}{T}\left(\frac{x}{r^{2}}\right)^{\frac{3-\theta_{0}}{2}} & \ll x^{-\alpha+\frac{3-\theta_{0}}{2}+\epsilon} \sum_{r<x^{\gamma}} r^{\beta-3+\theta_{0}} \\
& \ll\left\{\begin{array}{lll}
x^{\frac{3-\theta_{0}}{2}-\alpha+\epsilon} & \text { if } & 3-\theta_{0}-\beta>1, \\
x^{\frac{3-\theta_{0}}{2}-a+\gamma \theta_{0}-2 \gamma+\epsilon} & \text { if } & 3-\theta_{0}-\beta<1,
\end{array}\right.
\end{aligned}
$$

where $a=\alpha-\gamma \beta$. All the other error terms contribute a smaller amount. We leave them as an exercise.

Collecting all the error terms in (4.56), (4.57), (4.58), (4.59), (4.60), and (4.61), we see that if $\beta>\frac{8}{9}$ and $3-\theta_{0}-\beta<1$, then the total error is

$$
\mathcal{O}\left(x^{1-\gamma+\epsilon}+x^{\frac{1+\theta_{0}}{2}+\epsilon}+x^{\frac{9}{2} \alpha+\epsilon}+x^{\frac{3-\theta_{0}}{2}-a+\gamma \theta_{0}-2 \gamma+\epsilon}\right) .
$$

If we equalize these four error terms above, and solve in terms of $\theta_{0}$, it follows that

$$
\gamma=\frac{1-\theta_{0}}{9}, \quad \alpha=\frac{1+\theta_{0}}{9}, \quad a=0 \Longrightarrow \alpha=\gamma \beta
$$

The condition $3-\theta_{0}-\beta<1$ implies that $\beta>2-\theta_{0}$ which implies that $\alpha=\gamma \beta>\gamma\left(2-\theta_{0}\right)$ which gives

$$
\frac{1+\theta_{0}}{9}>\frac{1-\theta_{0}}{2}\left(2-\theta_{0}\right) \text {. }
$$

These inequalities imply that $\theta_{0}>\frac{1}{18}(29-\sqrt{265})$. With this choice, the total error in (4.62) is $\mathcal{O}\left(x^{\frac{1}{36}(47-\sqrt{265})+\epsilon}\right)$, where $\frac{1}{36}(47-\sqrt{265}) \sim 0.853366 \ldots$ This completes the Proof of Theorem 1.1.

\section{Acknowledgements}

The authors would like to extend their warmest thanks to D. Bump, B. Conrey, S. Friedberg, P. Sarnak for many very helpful conversations.

Adrian Diaconu would like to thank AIM for its generous support of this research in the summer of 2001. The second two authors are partially supported by the National Science Foundation. 


\section{References}

[A] Atkinson, F. V.: The mean value of the Riemann zeta function, Acta Math. 81 (1949), 353-376.

[B-H] Brezin, E. and Hlikami, S.: Characteristic polynomials of random matrices, Comm. Math. Phys. 214 (2000), 111-135.

[B-F-H-1] Bump, D., Friedberg, S. and Hoffstein, J.: Sums of twisted GL(3) automorphic $L$-functions, Preprint.

[B-F-H-2] Bump, D., Friedberg, S. and Hoffstein, J.: On some applications of automorphic forms to number theory, Bull. Amer. Math. Soc. 33 (1996), 157-175.

[C-F] Conrey, J. B. and Farmer, D. W.: Mean values of L-functions and symmetry, Internat. Math. Res. Notices 17 (2000), 883-908.

[CFKRS] Conrey, J. B., Farmer, D. W., Keating, J. P., Rubenstein, M. O. and Smith, N. C.: Moments of Zeta and L-functions.

[C-G] Conrey, J. B. and Gonek, S. M.: High moments of the Riemann zeta-function, Duke Math. J. 107 (2001), 577-604.

[C-Gh-1] Conrey, J. B. and Ghosh, A.: A conjecture for the sixth power moment of the Riemann zeta-function, Internat. Math. Res. Notices 15 (1998), 775-780.

[C-Gh-2] Conrey, J. B. and Ghosh, A.: On mean values of the zeta-function, Internat. Mathematica 31 (1984), 159-161.

[C-N] Chandrasekharan, K. and Narasimhan, R.: Functional equations with multiple gamma factors and the average order of arithmetical functions, Ann. of Math. 76 (1962), 93-136.

[D] Davenport, H.: Multiplicative Number Theory, 3rd edn, Grad. Texts in Math. 74, Springer-Verlag, New York, 2000.

[F-F] Fisher, B. and Friedberg, S.: Double Dirichlet Series Over Function Fields.

[F-H] Friedberg, S. and Hoffstein, J.: Nonvanishing theorems for automorphic L-functions on GL(2), Ann. of Math. 142 (1995), 385-423.

[G] Good, A.: The convolution method for Dirichlet series. In: The Selberg Trace Formula and Related Topics (Brunswick, Maine, 1984), Contemp. Math. 53, Amer. Math. Soc., Providence, RI, 1986, pp. 207-214.

[G-H] Goldfeld, D. and Hoffstein, J.: Eisenstein series of 1/2-integral weight and the mean value of real Dirichlet $L$-series, Invent. Math. 80 (1985), 185-208.

[H] Hoffstein, J.: Eisenstein series and theta functions on the metaplectic group, In: M. Ram Murty (ed.), Theta Functions: from the Classical to the Modern, CRM Proc. Lect. Notes 1, Amer. Math. Soc., Providence, RI, 1993, 65-104.

[H-B] Heath-Brown, D. R.: A mean value estimate for real character sums, Acta Arith. 72 (1995), 235-275.

[H-L] Hardy, G. H. and Littlewood, J. E.: Contributions to the theory of the Riemann zeta-function and the theory of the distributions of primes, Acta Math. 41 (1918), 119-196.

[Hö] Hörmander, L.: An Introduction to Complex Analysis in Several Variables, Van Nostrand, Princeton, N.J., 1966.

[H-R] Hoffstein, J. and Rosen, M.: Average values of $L$-series in function fields, J. Reine Angew. Math. 426 (1992), 117-150.

[I] Ingham, A. E.: Mean-value theorems in the theory of the Riemann zeta-function, Proc. London Math. Soc. 27 (1926), 273-300.

[J] Jutila, M.: On the mean value of $L\left(\frac{1}{2}, \chi\right)$ for real characters, Analysis 1 (1981), 149161.

[K-S] Katz, N. M. and Sarnak, P.: Random Matrices, Frobenius Eigenvalues, and Monodromy, Amer. Math. Soc. Colloq. Publ., 45, Amer. Math. Soc., Providence, RI, 1999. 
[Ke-Sn-1] Keating, J. P. and Snaith, N. C.: Random matrix theory and $\zeta(1 / 2+i t)$, Comm. Math. Phys. 214 (2000), 57-89.

[Ke-Sn-2] Keating, J. P. and Snaith, N. C.: Random matrix theory and $L$-function at $s=1 / 2$, Comm. Math. Phys. 214 (2000), 91-110.

[K] Kubota, T.: On Automorphic Forms and the Reciprocity Law in a Number Field, Kinokuniya Book Store Co., Tokyo, 1969.

[Mot1] Motohashi, Y.: An explicit formula for the fourth power mean of the Riemann zeta-function, Acta Math. 170 (1993), 181-220.

[Mot2] Motohashi, Y.: A relation between the Riemann zeta-function and the hyperbolic Laplacian, Ann. Scuola Norm. Sup. Pisa Cl. Sci. 22(4) (1995), 299-313.

[Mot3] Motohashi, Y.: Spectral Theory of the Riemann Zeta Function, Cambridge University Press, Cambridge, 1997.

[S] Siegel, C. L.: Die Funktionalgleichungen einiger Dirichlestscher Reithen, Math. Zeit. 63 (1956), 363-373.

[So] Soundarajan, K.: Nonvanishing of quadratic Dirichlet $L$-functions at $s=\frac{1}{2}$, Ann. of Math. 152 (2000), 447-488.

[St] Stark, H. M.: Unpublished notes.

[T] Titchmarsh, E. C.: In: D. R. Heath-Brown (ed.). The Theory of the Riemann Zeta-function, 2nd edn, The Clarendon Press, Oxford University Press, New York, 1986.

[Z] Zavorotny, N. I.: Automorphic Functions and Number Theory, Parts I, II (Russian), Akad. Nauk SSSR, Dal'nevostochn. Otdel., Vladivostok (1989), 69-124a, 254. 